\title{
The Effect of a Six Week Functional Training Program on Performance Outcomes in Softball
}

\author{
Zachary M. Mohondro
}

Follow this and additional works at: https://researchrepository.wvu.edu/etd

\section{Recommended Citation}

Mohondro, Zachary M., "The Effect of a Six Week Functional Training Program on Performance Outcomes in Softball" (2014). Graduate Theses, Dissertations, and Problem Reports. 7332.

https://researchrepository.wvu.edu/etd/7332

This Thesis is protected by copyright and/or related rights. It has been brought to you by the The Research Repository @ WVU with permission from the rights-holder(s). You are free to use this Thesis in any way that is permitted by the copyright and related rights legislation that applies to your use. For other uses you must obtain permission from the rights-holder(s) directly, unless additional rights are indicated by a Creative Commons license in the record and/ or on the work itself. This Thesis has been accepted for inclusion in WVU Graduate Theses, Dissertations, and Problem Reports collection by an authorized administrator of The Research Repository @ WVU. For more information, please contact researchrepository@mail.wvu.edu. 
The Effect of a Six Week Functional Training Program on Performance Outcomes in Softball

\author{
Zachary M. Mohondro, ATC
}

Thesis submitted to the

College of Physical Activity and Sport Sciences

at West Virginia University in partial fulfillment of the requirements

for the degree of

\author{
Masters of Science \\ in \\ Athletic Training \\ Michelle A. Sandrey, PhD, ATC, Chair \\ Emidio E. Pistilli, PhD \\ Drue T. Stapleton, PhD, ATC, CSCS \\ Department of Sport Sciences
}

Morgantown, WV 2014

Keywords: Functional training, throwing accuracy, overhead throwing, softball 
All rights reserved

INFORMATION TO ALL USERS

The quality of this reproduction is dependent upon the quality of the copy submitted.

In the unlikely event that the author did not send a complete manuscript and there are missing pages, these will be noted. Also, if material had to be removed, a note will indicate the deletion.

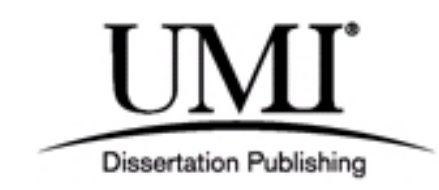

UMI 1555070

Published by ProQuest LLC (2014). Copyright in the Dissertation held by the Author.

Microform Edition () ProQuest LLC.

All rights reserved. This work is protected against unauthorized copying under Title 17, United States Code

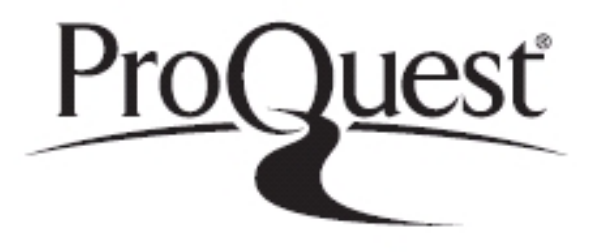

ProQuest LLC.

789 East Eisenhower Parkway

P.O. Box 1346

Ann Arbor, MI 48106 - 1346 


\begin{abstract}
The effects of a Six Week Functional Training Program on Performance Outcomes in Softball Zachary M Mohondro
\end{abstract}

Context: Functional training for overhead throwing in softball is an area that has yet to be explored in research. Despite inclusion of functional training in current rehabilitation and strength and conditioning programs, few studies have examined the implementation of progressions, and the benefits therein. Objective: The objective of this study was to determine the effectiveness of a functional training program on performance outcomes. Design: This study was a 2 × 2 factorial design with group (experimental and control) and time (pre and post). This was a case control repeated measures design. The dependent variables are the functional training performance index, closed kinetic chain upper extremity stability test, joint position test, and throwing velocity test. Setting: NCAA Division III college in western Pennsylvania. Patients and Other Participants: Experimental subjects consisted of 21 healthy female softball athletes from a Division III collegiate setting. There were 11 subjects in the experimental group and 10 subjects in the control group. Subjects were put into groups using stratified randomization. Stratification was based on position of infield, outfield, catchers, and pitchers. Inclusion criteria were: healthy subjects who have not had shoulder, hip, or abdomen surgery within the year and have not had a shoulder, hip, or abdominal injury in the past six months. Exclusion criteria included those who have had shoulder, hip or abdominal surgery within the past year and those who have had a shoulder, hip or abdominal injury within the past six months. Interventions: Those that received functional training completed a six week functional training progression aimed at improving throwing accuracy, throwing velocity, and proprioception. Pre and post-test measurements for throwing accuracy using the functional throwing performance index, upper extremity proprioception using the closed kinetic chain upper extremity stability test and joint position sense, and upper extremity power using the throwing velocity test were conducted following a six week functional training progression. The progression was administered twice a week for an average of 30 minutes each session. The kinetic chain progression of exercises were advanced from week to week and incorporated open kinetic chain exercises, closed kinetic chain exercises, plyometric exercises, and functional exercises. Main Outcome Measures: The dependent variables were average index scores from the functional training performance index, average throwing velocity, average absolute total error measured by the joint position test, and average number of touches from the closed kinetic chain upper extremity stability test. Results: Joint position sense testing showed no significant interaction, time, arm or group outcomes. No measure exceeded the minimal detectable change. Throwing accuracy testing showed a significant group interaction $(\mathrm{P}=0.033)$. There was a large effect size between groups $(\mathrm{ES}=-1.02)$ in favor of the experimental group. The experimental group exceeded the minimal detectable change for throwing velocity. The closed kinetic chain upper extremity stability test showed a significant difference from pre to post test $(\mathrm{P}=0.002)$, and both the control and experimental group exceeded the minimal detectable change. Conclusion: A multi-segment training program may be used to improve throwing velocity and throwing accuracy. The trend of the data is consistent with the hypotheses, and the results support its use in the clinical setting. This program has shown that it can be implemented into the clinical setting. Further research is warranted to ascertain the efficacy of functional training among a larger population. 


\section{ACKNOWLEDGEMENTS}

First and foremost, I would like to extend my gratitude, love, and appreciation to my family: Dad, Mom, RJ, Jordan, and Shawna. You have always been there to support me and no matter what, I know that I have you to rely on. Family is the most important thing to me, and I hope that I make you proud. I love you all.

I would like to thank my friends Bert, Andrew, Jeff, Ryne, and Justin. I have known most of you since we were in grade school and your support through my life, both good times and bad, have been unbelievable. I cannot express into words the impact each of you has made on me.

I would like to thank the Debbie, Brian, Andrew, and Anna Fitzsimmons. You have been so instrumental in my life and have done so much to help carry me through all the hard times. Thank you for always being there and you have taught me so much. I owe my success to you as much as anyone else.

I would like to thank Dr. Michelle Sandrey for your support, guidance and most of all your patience. You have played an instrumental part in my professional development, and I know my two years at WVU will serve me well in my future career. I would like to thank you for the hours spent with me describing and directing me to develop my thesis and classwork. Without your insight, I wouldn't be where I am today.

I would like to thank Dr. Drue Stapleton. Though I have only known you for less than a year, you have made quite an impression on me. Your leadership, knowledge, and poise have inspired me. You have served as a professional role model for me this past school year. Thank you for taking the time to be on my thesis committee despite the amount of responsibility you have. Your thoughtful discussions and wisdom furthered my understanding and gave me confidence in abilities. Thank you for all your help and encouragement through this process, and it has been a pleasure working with you.

I would like to thank Dr. Emidio Pistilli. Despite the fact that I approached you only a few weeks before my thesis proposal meeting, you were completely willing to be a part of this process. I want to thank you for all the help you have given me and your accommodating disposition.

I would like to thank the Waynesburg University Softball team and coaches. Their compliance and hard work throughout this study was singular. Thank you to the coaches for allowing me to use the team even though it was in the middle of the season. Good luck next year. 


\section{TABLE OF CONTENTS}

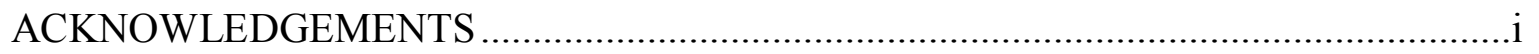

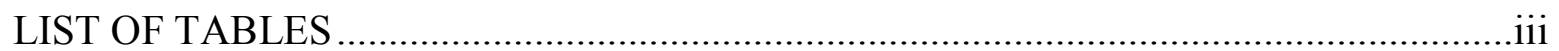

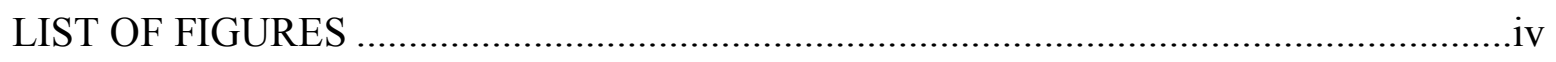

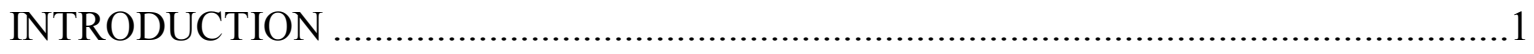

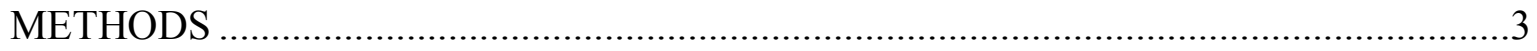

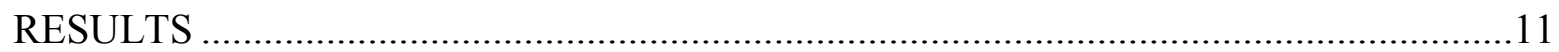

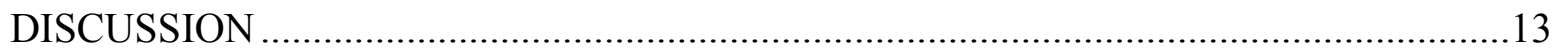

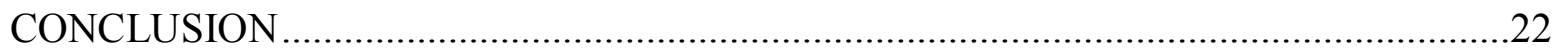

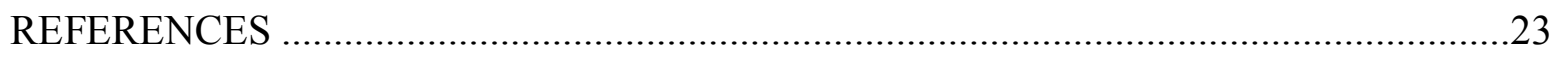

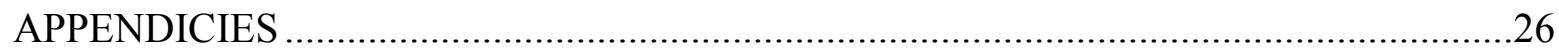

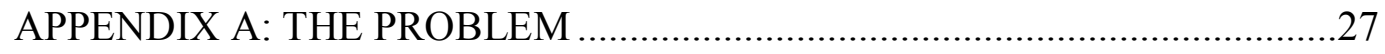

APPENDIX B: LITERATURE REVIEW ……………......................................

APPENDIX C: ADDITIONAL METHODS ......................................................63

APPENDIX D: ADDITIONAL RESULTS ……………..................................

APPENDIX E: RECOMMENDATIONS FOR FUTURE RESEARCH ..............102

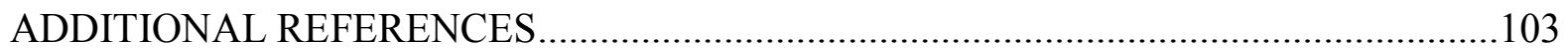




\section{LIST OF TABLES}

Table C1. INFORMED CONSENT ........................................................................63

Table C2. SUBJECT DEMOGRAPHICS ................................................................69

Table C3. PRE POST DATA COLLECTION SHEET …............................................. 70

Table C4. FUNCITONAL TRAINING PROGRESSION .............................................71

Table D1. DESCRIPTIVE STATISTICS OF MEANS AND STANDARD DEVIATIONS FOR

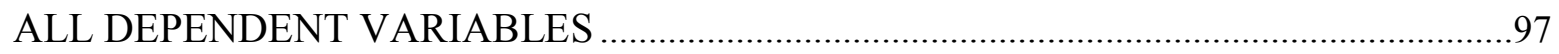

Table D2. MINIMAL DETECTABLE CHANGE VALUES ..........................................98

Table D3. PAIRWISE COMPARISON FOR PRE AND POST TEST ............................99

Table D4. PAIRWISE COMPARISON FOR EXPERIMENTAL GROUP AND CONTROL

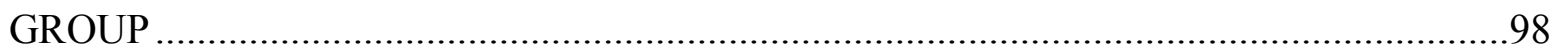




\section{LIST OF FIGURES}

Figure C1. PRETEST/POSTTEST MEASURES ........................................................ 73

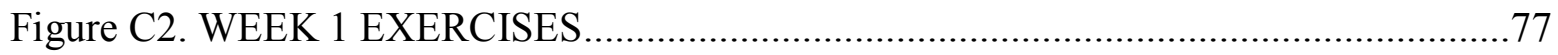

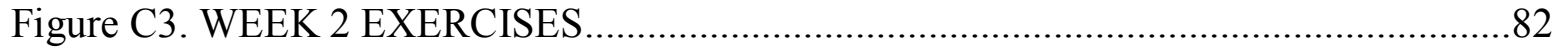

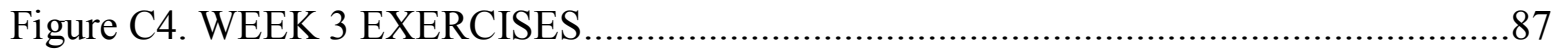

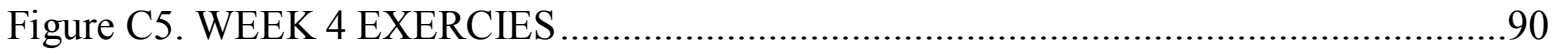

Figure C6. WEEK 5 EXERCISES.................................................................. 93

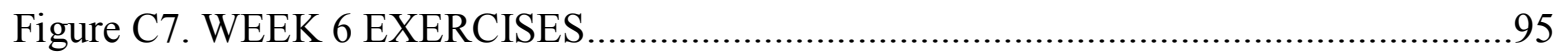

Figure D1. LEFT ARM JOINT POSITION PLOT .....................................................99

Figure D2. RIGHT ARM JOINT POSITION SENSE PLOT ….....................................99

Figure D3. FUNCTIONAL THROWING PERFORMANCE INDEX PLOT .....................100

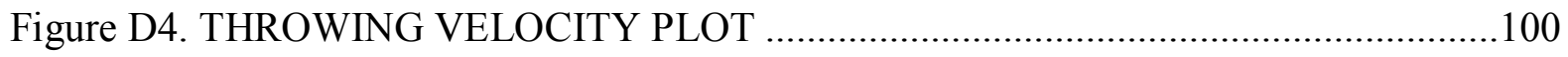

Figure D5. CLOSED KINETIC CHAIN UPPER EXTREMITY STABILITY TEST PLOT 


\section{INTRODUCTION}

The glenohumeral joint is a highly unstable joint that provides a unique balance between stability and mobility. It relies heavily on dynamic stabilization to properly function, ${ }^{1}$ but also allows for a large amount of force to be transferred during dynamic movement, perhaps most aptly displayed in overhead throwing. Specific and sequential movement of all body parts are necessary for these skills in order to perform effective and safe movements. ${ }^{2}$ This linkage between all parts of the body, known collectively as the 'kinetic chain', provides for the transfer of those forces produced proximally to be funneled distally to the extremities. The lower extremity, trunk, and shoulder are the three main segments of the throwing motion, and the integration into a functional movement pattern is vital for safe and efficient movement. Forces generated at the hips and trunk can be transferred through the shoulder and to the ball, resulting in a high velocity throw. ${ }^{2,3,4,5}$ These forces, however, need to be directed in a controlled manner, requiring a great deal of neuromuscular control in order to sequence the contraction of musculature in a highly specific pattern that results in a directed throw while still maintaining the stability of the joints involved. The highly coordinated nature of the overhead throwing motion and the inherent role of the kinetic chain make it a highly complex skill, and abnormal mechanics may put athletes at risk and result in injury.

Softball is a growing sport that has millions worldwide participating both competitively and recreationally. ${ }^{6,7,8}$ In the high school setting, it has become one of the most popular sports, ranking fourth as of 2004. ${ }^{9}$ It is a highly complex sport, requiring a multitude of different skills and extreme movements, especially overhead throwing. There has been a similar reported rate of injury for girls in softball than for boys participating in baseball. ${ }^{8}$ Thus, rehabilitation of softball athletes have followed programs and research developed for baseball. Because the similarities 
between baseball and softball run quite deep, it has been generally assumed that rehabilitation efforts for baseball can be extrapolated and be effective for softball rehabilitation programs. As such, the development of rehabilitation strategies for softball is heavily based on concepts from research focused on baseball rehabilitation.

Given that softball is an extreme sport with a high incidence of injury, it stands to reason that a proper strength and conditioning is necessary for the success of an athlete to complete the movements safely without predisposing them to injury. However, the lack of attention to softball extends beyond rehabilitation. Strength and conditioning programs specific to softball are rare in the literature, and most are, again, borrowed from concepts in baseball. Most strength and conditioning programs are basic in nature, and only advocate for a basic weight training regimen along with conditioning exercises. ${ }^{10,11}$ Other studies ${ }^{12,13,14,15}$ have shown the efficacy of specific types of exercises over another, but have not demonstrated the benefit in terms of applied functional outcomes related to throwing. The overhead throwing motion is a complex movement that requires a high degree of control and coordination between proximal and distal body segments, and training should reflect this concept of incorporating the kinetic chain. The appearance of functional training progressions in strength and conditioning programs is lacking in the literature. To date, there has not been a functional progression published for the sake of strength and conditioning for softball.

The development of a functional training program should reflect the movement patterns used in softball. Specifically, the overhead throwing motion in softball and the biomechanical principles that underlie it should guide how a functional training program is created. Following progressive strengthening, diagonal patterns and proximal-to-distal muscle activation patterns that are characteristic of an overhead throw should be incorporated in a progressive functional 
training program as well as the types of muscle contractions used in the throwing motion. Thus, the following concepts have been used in a functional training program to evaluate its efficacy outside of a laboratory setting. Therefore, the purpose of this study is to examine the effect a functional training program that is based on biomechanical principles of the overhead throw has on performance outcomes.

\section{METHODS}

This study was a case-control repeated measures design. The independent variables were test and group. The testing was time dependent in the manner of a pre-test and post-test protocol. The groups were categorical and existed on two levels of a control group and experimental group. The dependent variables consisted of the Joint Position Sense Test absolute angle error, , the Functional Throwing Performance Index Test accuracy scores, the Throwing Velocity Test average miles per hour, and the Closed Chain Upper Extremity Stability Test scores. The Functional Throwing Performance Index was measured by an index score, calculated by dividing the number of throws taken by the number of accurate throws. This study was approved by the Institutional Review Board.

Subjects

The experimental group in this study included 11 collegiate female softball players from a Division-III institution in western Pennsylvania using a sample of convenience. The average age of the subjects in this group was 20.2 years, the average weight was 71.5 kilograms, and the average height was $167.6 \mathrm{~cm}$. The group included pitchers, catchers, infield and outfield players. Inclusion criteria included those subjects who are healthy, have no disorders affecting balance or neuromuscular control, no history of an injury to the shoulder in the past six months, no history of surgeries to the upper extremity, core, or hips within the past year, and those taking 
medication that may affect balance that could skew results. Exclusion criteria includes subjects who have a history of surgery to the upper extremity, core, or hips within the past year, an injury to the shoulder within the past six months, those who have a disorder affecting balance and neuromuscular control, and those taking medication that may affect balance and neuromuscular control. To remain in the study, experimental subjects must complete $75 \%$ of the intervention $(9$ sessions). One subject in the experimental group was unable to complete the exercises due to injury. All subjects in this group were right hand dominant. All experimental subjects completed at least 9 training sessions.

The control group included 10 subjects from the same softball team. The average age of the control group was 19.8 years, the average weight was 68.4 kilograms, and the average height was $166.8 \mathrm{~cm}$. Inclusion criteria included those subjects who were healthy, had no disorders affecting balance or neuromuscular control, no history of an injury to the glenohumeral joint complex in the past six months, no history of surgeries to the upper extremity, core, or hips within the past year, and not taking medication that may affect balance that could skew results. Exclusion criteria included subjects who have a history of surgery to the upper extremity, core, or hips within the past year, an injury to the shoulder within the past six months, disorders, those who have a disorder affecting balance and neuromuscular control, and those taking medication that may affect balance and neuromuscular control. To remain in the study, all control subjects must complete $100 \%$ of the pre and posttest measures. One subject in the control group was unable to complete the posttest measures due to injury. All subjects in this group were right hand dominant. 
Procedures

Before commencing the pre-testing procedures and beginning the functional training program, an orientation meeting took place with the subjects. In this meeting, the informed consent form with HIPPA included (Table C1) and the demographic questionnaires (Table C2) were discussed. The informed consent with HIPPA and demographic questionnaire was completed during this orientation session. Once subjects were determined, testing procedures (Table C3) as well as the functional training progression (Table C4) were discussed as well. Instructions for the testing procedures were given to all subjects in both the experimental and control groups (Figure C1). In addition, instructions for the exercises in the functional progression were provided for those subjects in the experimental group (Figures C2-C7). It was also explained that, during testing days and days in which exercises are to be performed, subjects are not to participate in any personal workouts that includes cardiovascular training and strength training. It was also explained that during throughout the duration of the study, all cardiovascular training and strength training should be kept at a minimum and any activity outside of the assigned training program should be reported to the principal investigator for documentation. These guidelines applied for the control group as well.

Following the orientation meeting, all subjects completed pre-testing procedures in order to establish baseline values. Pre-testing procedures included the Joint Position Sense Test, the Functional Throwing Performance Index, the Throwing Velocity Test, and the Closed Chain Upper Extremity Stability Test. Prior to all testing, procedures and instrumentation were described to the subject. The researcher performed all testing and training procedures. A warm up time of five minutes on a treadmill at a self-selected pace was performed on each subject 
followed by five minutes of stretching of the rotator cuff, biceps, pectoralis major, pectoralis minor and triceps of the throwing shoulder performed by the principal investigator.

Following the pre-testing procedures, all experimental subjects participated in the functional training progression (Table C4). Exercises for each week were performed twice per week, with each session depending on the patient's availability during that week. Sets and repetitions per set for each exercise can be found in Table C4. In this program, progression was based on difficulty of exercise as opposed to an increase in weight or repetitions in order to target the integration of exercises from isolated segments to full body movement thereby enhancing the efficiency of the kinetic chain.

Pre-test / Post test Procedures (Figure C1)

Joint position sense: The subject then performed the Joint Position Test. This tested the subject's kinesthetic awareness in the shoulder. The subject laid on their back on a table in the supine position with their eyes closed. The researcher moved subject's arm to a position of ninety degrees of glenohumeral abduction and ninety degrees of glenohumeral internal rotation using a goniometer and asked the subject to remember where the arm is in space. The primary investigator then moved the arm back to a resting position at the side. The subject will then attempted to move the arm back to the testing position. The researcher measured the subject's arm position using a goniometer. The researcher recorded the absolute value of the error for glenohumeral rotation. These procedures were repeated three times and the average error was used (Table C3). This test was performed for both the right and left arms of each subject. ICC calculation for the right arm was 0.429 . ICC calculation for the left arm was 0.318 .

Functional throwing performance index: ${ }^{15}{ }^{16}$ Following the joint position test, the subject performed the Functional Throwing Performance Index Test. The subject was instructed to stand 
15 feet away from a $1 \mathrm{ft} \times 1 \mathrm{ft}$ square taped on a wall that is $4 \mathrm{ft}$ off the ground. The subject attempted to throw the softball into the square, grab the rebound, and repeat throwing the ball into the square using a natural throwing motion. The subject was given the chance to practice the test with a $25 \%, 50 \%$, and $75 \%$ submaximal warm up throws, each lasting 30 seconds. The subject performed the test and threw as many times into the square as many times as possible in 30 seconds. This test was repeated three times and the average score was used. The index score is calculated by dividing the number of throws taken by the number of accurate throws that strike the target. The researcher recorded the score for the subject. (Table C3). There was a five minute rest period before the next test. The functional performance throwing index has shown good intrasession reliability, ${ }^{15}$ and in this study the ICC was shown to be 0.460 .

Throwing velocity test: ${ }^{17}$ The Throwing Velocity Test was performed as the subject stood twenty feet away from a wall. The subject warmed up and threw a softball at $25 \%, 50 \%$, and $75 \%$ the effort. The subject then performed three throws at full effort. The researcher stood behind the throwing line with a radar gun (JUGS pro-sports digital radar, R2000, JUGS sports, Tualatin, OR) and measured the velocity of the throw. The average velocity of the three throws was used and the researcher recorded all results (Table C3). There was a five minute rest period before the next test. ICC calculation was 0.881 .

Closed kinetic chain upper extremity stability test: ${ }^{16,18}$ The Closed Chain Upper Extremity Stability Test was performed with the subject assuming a push-up position with the hands 36 inches apart and the hands placed on a piece of tape 1.5 inches in length. The subject removed the non-dominant hand and placed it next to the dominant. The subject then re-placed the hand back to the starting position and then removed the dominant hand and placed it next to the non-dominant hand. A touch was defined as crossing the midline and touching the opposite 
piece of tape. The subject repeated this sequence as many times as they can in 15 seconds for three trials. The average number of touches between the trials was used, and the principal investigator recorded all scores (Table C3). Once the number of touches was recorded, the data was normalized by dividing by the patient's height. This has been shown to be a reliable evaluation tool. ${ }^{18}$ ICC calculation was 0.907 .

Intervention Program

All exercises included in the intervention program were adapted from clinical recommendations and research. Plyometric exercises were recommended from Pezzullo et al., ${ }^{19}$ Carter et al, ${ }^{17}$ Burkhart et al,${ }^{20}$ Padua et al,${ }^{15}$ and Borsa et al. ${ }^{21}$ Closed Kinetic Chain exercises and open kinetic chain exercises were recommended from Prokupu et al. ${ }^{12}$ Other functional exercises were utilized and modified from Kibler et al, ${ }^{2}$ Sciascia et al,${ }^{4}$ and Palmer et al. ${ }^{22}$ Lower extremity functional exercises were adapted from Crotin et al. ${ }^{23}$ Core stability exercises were developed and adapted from Lust et al, ${ }^{16}$ and Palmer et al. ${ }^{22}$

Week 1 (Figure C2): ${ }^{2,16,19,20}$ During the first week, the subject performed Y's, T's, 90/90 Thera-band external rotation, 90/90 Thera-band internal rotation, quadruped (single limb), single leg bridges, hip 4-way, front lunges, and side lunges. These exercises served to strengthen the isolated segments of the throwing motion in order to provide stable joint mechanics to prepare for more complex motions.

Week 2 (Figure C3): ${ }^{15}, 16$ During the second week, the subject performed $D_{1}$ and $D_{2}$ shoulder Proprioceptive Neuromuscular Facilitation (PNF) exercises with manual resistance from the principal investigator. They also performed rhythmic stabilization, quadruped (double limb alternating), single leg bridges on airex, front BOSU lunges, side BOSU lunges, and BOSU 
Squats. These exercises were progressions of complexity to those in week 1 and also enhanced stability of the core.

Week 3 (Figure C4): ${ }^{15,16,23}$ During the third week the subject performed BOSU pushups, physioball push up balance, leg lifts with rotation, double leg bridges on physioball, medicine ball lateral swing hop, and the lateral ground hop. These exercises were further progressions of complexity, but also incorporated multi-planar and multi-segment movement to begin to integrate functional patterns through the body. This week advanced from the previous weeks by incorporating plyometric movement patterns and closed kinetic chain exercises in order to stimulate proper muscle and mechanoreceptor action. ${ }^{14,15,20}$

Week 4 (Figure C5): ${ }^{16,19,22}$ The fourth week contained exercises that included the kneeling 90/90 ball toss forward, kneeling reverse throw, kneeling chop, medicine ball rebound lateral toss, front drop back lunge, and the plyometric lunge ground hop. These exercises were progressions from those seen in week 3 and also incorporated explosive movement patterns similar to those in the overhead throw. ${ }^{19}$

Week 5 (Figure C6): ${ }^{5,24}$ The fifth week started full-body dynamic movements. The exercises in this week were lunge punches, lawnmower pulls, and stride balance with rotation. These were the start of full body movements that further integrated all three segments of the throwing motion in functional patterns.

Week 6 (Figure C7): ${ }^{2}$ The last week was a progression of the fifth week. The exercises in the sixth week most resembled the movement patterns in the overhead throwing motion. The exercises in this week were the life/place with dumbbell, standing lunge chop with rotation, and the thera-band throwing motion with rotation. These exercises incorporated all aspects of the throwing motion into a single, dynamic motion. 
Post-testing porocedures followed the conclusion of the functional training program. These testing procedures were the same as the pre-testing procedures (Figure C1). Data was collected by the principal investigator (Table C3). The researcher then analyzed and compared the pre-test data and the post-test data.

Statistical Analysis

Descriptive statistics were calculated for both the control group and experimental group. Descriptive analysis included pre and posttest means and standard deviations for the functional throwing performance index scores, joint position sense absolute angle error, closed kinetic chain upper extremity stability test touches, and throwing velocity averages. Further statistical analysis used a 2 X 2 repeated measures ANOVA for throwing velocity, throwing accuracy amd the closed kinetic chain upper extremity stability test. A 2 X 2 X 2 repeated measures ANOVA was used for joint position sense testing. $\mathrm{P}$-values for all analyses were set at $\mathrm{P}=0.05$. To measure effect size, Cohen's $d$ was calculated based on the mean differences of pretest and posttest scores divided by the pretest standard deviation, which served as the reference standard deviation. There was a 95\% confidence interval used. Small effect size was defined as $\leq .4$. Moderate effect size was defined as .41-.7, and large effect size was defined as $\geq .71 .{ }^{25}$ Minimal detectable change (MDC) was defined as the minimal change in the scores of the dependent variables that surpass the error measurement. These were calculated using intraclass correlation coefficients and the standard error. ${ }^{26}$ All statistical analyses were conducted using IBM SPSS statistical analysis software (IBM version 20 Chicago, Il). 


\section{RESULTS}

Joint Position Sense

Joint position sense testing revealed no significant interactions for time by group by arm $(\mathrm{F}=0.306, \mathrm{P}=0.588)$, time by arm $(\mathrm{F}=0.243, \mathrm{P}=0.628)$, arm by group $(\mathrm{F}=4.086, \mathrm{P}=0.059)$, or time by group $(\mathrm{F}=0.538, \mathrm{P}=0.461)$. There also were no significant differences in time $(\mathrm{F}=0.953$, $\mathrm{P}=0.343)$, group $(\mathrm{F}=0.418, \mathrm{P}=0.527)$, or arm $(\mathrm{F}=1.224, \mathrm{P}=0.284)$. Effect sizes were evaluated and showed a poorer performance for both arms in the control group between pre and posttest. The experimental group had a small effect size in the left $(\mathrm{ES}=0.27)$ and right arm $(E S=-0.17)$ from pre to posttest. There was a large effect size was evident between groups in the right arm $(E S=-0.88)$, but not in the left $(E S=0.25)$. All descriptive statistics can be found in Table D1 and graphs can be found in Figure D1 and Figure D2.

MDC for the right arm was calculated at 5.728, while the MDC for the left arm was calculated at 4.454. No differences between pre and post measurements for the right arm for either group exceeded the MDC ( $\Delta$ Control $=-1.4822, \Delta$ Experimental $=0.8)$. No differences between pre-test and post-test measurements for the left arm for either group exceeded the MDC ( $\Delta$ Control=-1.370, $\Delta$ Experimental $=-1.1674)$. These can be found in Table D2.

Throwing Accuracy

There was a significant difference between groups $(\mathrm{F}=5.386, \mathrm{P}=0.033)$ with the experimental group $(\mathrm{P}=0.033)$ performing better than the control group. Throwing accuracy showed no significant interactions for time by group $(\mathrm{F}=1.161, \mathrm{P}=0.296)$ or time $(\mathrm{F}=0.406$, $\mathrm{P}=0.533$ ). The control group showed a decreased performance in the test from pre-test to posttest with a small effect size $(\mathrm{ES}=0.11)$. The experimental group showed a large effect size $(\mathrm{ES}=$ 0.77) between pre-test and post-test measures. There was a large effect size between groups 
during post-test procedures as well $(E S=-1.02)$ that was also statistically significant. All results can be found in Table D1 and a graph can be found in Figure D3.

MDCs for the Functional Throwing Performance Index was calculated at 0.460 . No differences between pre-test and post-test measurements for either group exceeded the MDC ( $\Delta$ Control $=-0.0511, \Delta$ Experimental=0.229). Results can be found in Table D2.

Throwing Velocity

Throwing velocity testing did now have any significant interactions for time by group analysis $(\mathrm{F}=3.915, \mathrm{P}=0.064)$. There were no significant differences for time $(\mathrm{F}=1.071, \mathrm{P}=0.315)$ or group $(\mathrm{F}=0.187, \mathrm{P}=0.671)$. There was a decrease in performance in the control group from pretest to post-test $(E S=-0.10)$, but the experimental group showed a moderate effect size from pretest to post-test $(\mathrm{ES}=0.58)$. There was also a moderate effect size between groups as well (ES=0.41), with the experimental group performing better. Results can be found in Table D1 and a graph can be found in Figure D4.

MDC for the throwing velocity test was calculated at 1.948 . The control group did not exceed the MDC. However, the experimental group improved pre-test and post-test difference did exceed the MDC ( $\Delta$ Control= -0.6678, $\Delta$ Experimental=2.133). These can be found in Table D2.

\section{Closed Kinetic Chain Upper Extremity Stability Test}

The closed kinetic chain upper extremity stability test revealed that there was a significant difference evaluated for time measurements $(F=14.111, p=0.002)$. There were no differences found with time by group measurements $(\mathrm{F}=0.061, \mathrm{P}=0.807)$ or group $(\mathrm{F}=0.057$, $\mathrm{P}=0.814)$. From pre-test to post-test the control group had a moderate effect size $(\mathrm{ES}=0.50)$ and 
the experimental group had a large effect $(0.75)$. There was no effect size for group $(\mathrm{ES}=0)$. Results can be found in Table D1 and a graph can be found in Figure D5.

MDC was calculated at 0.02106. Both the control and experimental group's pre-test and post-test differences exceeded the MDC $\quad(\Delta$ Control $=0.026214, \Delta$ Experimental $=0.0299168$ ). Results can be found in Table D2.

\section{DISCUSSION}

The purpose of this study was to assess the efficacy of a sport-specific, functional training program on performance measures. Measures assessed included joint position sense, throwing accuracy, throwing velocity, and upper extremity neuromuscular control. Joint position sense was measured using a standard goniometer, throwing accuracy measured by the functional

throwing performance index, ${ }^{15,16}$ the throwing velocity was measured by a JUGS radar gun, and the upper extremity neuromuscular control was measured by the closed kinetic chain upper extremity stability test. ${ }^{16,18}$

It was hypothesized that those subjects in the experimental group who underwent the six week training program would have significant improvements across all dependent variables as compared to the control group. Statistically significant results included the difference between groups for throwing accuracy with the experimental group performing better than the control group. Also, improvement from pretest to posttest for the closed kinetic chain upper extremity stability test was evident. Effect size calculations were consistent with the expected outcomes as well, most notably a large effect size between groups for throwing accuracy. A functional training program utilizes the concept of the kinetic chain to link multiple body segments together into one functional unit, ${ }^{2}$ and in doing so, allow for more efficient body movement that manifests itself in the form of increased performance measures. The intervention in this study utilized this 
concept in order to enhance the neuromuscular framework that allowed the kinetic chain to be utilized efficiently. The progression of the exercises allowed the individual components to be trained in a progressive, functional way and then subsequently combined in order to produce a proper overhead throwing motion involving all components of the kinetic chain. By doing so, it was found that there were clinically-relevant changes in throwing accuracy, throwing velocity, and neuromuscular control. This program was designed specifically for overhead throwing motion in softball, but there is the potential for it to be implemented or combined for the general overhead throw. More research is needed to further evaluate the efficacy as a training program designed to increase throwing accuracy and throwing velocity.

Joint Position Sense

Kinesthetic sense represents the proprioceptive input brought on by mechanoreceptors that provide unconscious or conscious awareness of joint position. ${ }^{27}$ The results of this study indicated that a functional training program may not have an effect on joint position sense. Even though there was a large effect size between groups in the dominant arm, there were not any exercises that were specific to improving joint position sense. Within the training program used in this study, the participants in the experimental group were subjected to exercises demanding the use of multiple segments, especially in the later weeks. The exercises used in this program were based on movement patterns rather than having a goal of increasing strength or power. The subjects were tasked to perform these specific movements, thereby creating a greater conscious awareness of their own kinesthetic sense globally rather than in the shoulder specifically. The goal is for the subject to gain awareness of the whole movement; so that movement patterns can be performed successfully. ${ }^{5}$ There was also a small effect size for the experimental group across time. This could have been the result of the exercises as well, but a longer training program may 
improve these results further. The lack of specific exercises targeted at joint position sense may have mitigated the effect size of time for the right arm of the experimental group.

The subjects used multiple segments in a synergistic manner, and performed the exercises using the kinetic chain. By doing so, adjacent segments to the glenohumeral joint complex can facilitate the activation of involved muscles to develop appropriate shoulder motion and function. ${ }^{4}$ The increased activation of shoulder muscles via synergistic activation may very well play a role in increasing joint position sense by enhancing the feed forward mechanism.

One other feature of the training program was the use of closed kinetic chain exercises. The use of these types of exercises have been reported to stimulate mechanoreceptors in the glenohumeral joint complex, ${ }^{14}$ eliciting a neuromuscular response. ${ }^{12}$ Joint approximation from these exercises is believed to stimulate peripheral afferent activity, stimulating reflexive muscle stabilization. ${ }^{15}$ The use of these exercises could have played a role in increasing joint position sense, although the extent to which is unclear, as the limbs being used in these exercises resulted in conflicting results in the experimental group, with the left arm having greater error and the right arm having a small effect size. It must also be noted that the subjects had intact neuromuscular systems as none reported injuries to the glenohumeral joint complex. There may have been little to gain from the training program.

Overall, the large effect size between groups may indicate that there may have been some proprioceptive changes occurring, although it is unclear due to the fact that the confidence intervals crossed zero and neither group exceeded the MDC. However, it is unknown what mechanisms may cause this change, or what length of time is needed to cause a change. 
Functional Throwing Performance Index

Throwing accuracy is a task that is modulated by many different inputs. Accuracy depends on proper muscle activation. A sequence of proximal to distal muscle activation is evident in overhand throwing. ${ }^{28,29}$ This proximal to distal activation sequence links all the segments of the throwing motion, thereby creating one functional unit, thus, decreasing the degrees of freedom in the motion by eliminating unnecessary movement patterns. ${ }^{2}$ By enhancing the kinetic links within the throwing motion, decreased error would ensue and better accuracy would result. Development of the neuromuscular framework allows for the hips, trunk, and shoulder to work in conjunction and in a specific pattern so as to perform a task and propel a ball forward with the distal segments with efficiency. ${ }^{2,28}$ The efficient movement allows for forces to be directed and modulated properly and safely to propel a ball to the desired location. The results of this study also indicated that this may be the case. There was a large effect size between groups with the experimental group doing better for the functional throwing performance index that was also statistically significant. This demonstrated that the proper integration of multiple segments results in greater control through a dynamic motion.

The training of proper movement patterns may play a role in the increased accuracy as well. The overhand throw is a complex motion, and every muscle involved must function in a specific proximal to distal manner. ${ }^{29}$ Allowing proximal stability to increase distal mobility is another benefit the kinetic chain provides. ${ }^{2}$ The results of this study are consistent with those seen in others as well. Padua et $\mathrm{al}^{15}$ found that throwing accuracy increased in subjects performing shoulder PNF exercises, another program that is designed to accentuate this proximal-to-distal muscle activation pattern. The results of this study further support these previous findings. Based on this proximal to distal sequencing, the arm ultimately depends on the 
proximal segments for movement. ${ }^{5}$ Synergistic action from the hips and trunk facilitate distal segments, ${ }^{4}$ which was an emphasis of this program. The incorporation of proximal segments and the integration of them with distal segments intended to facilitate proper movement and muscle function that would result in more accurate throws. Again, this was evident in the large effect size between groups as well as through time.

Compared to those without training, the experimental group was able to coordinate the entire motion and produce more efficient muscle control and decreased the variability of the kinetic chain within the throwing motion. This resulted in greater control that is shown through increased accuracy scores. Moreover, the increase across time suggested that deficiencies in the kinetic chain can be trained and reeducated to increase performance.

Throwing Velocity

Throwing velocity is a product of the amount of force produced by the musculature and how it is funneled distally. The kinetic chain allows for maximum force to be produced in the proximal segments and for them to be transferred into the hand. ${ }^{2}$ Again, the training program used in this study attempted to connect all the segments of the throwing motion in order to maximize the force output placed on the ball, which was displayed in the moderate effect size between groups as well as the moderate effect size from pre-test to post-test.

It is speculated that changes in the experimental group verify that training to enhance the kinetic chain and marginalize the variability therein allows for more force output to be generated in the throw. The ultimate velocity of the distal segment is dependent on the velocity of the proximal segments and the interaction of these segments, ${ }^{5}$ and as such the proper linkage of these segments maximizes the force output on the ball. Proper use of the kinetic chain permits maximal force to be developed throughout the body so that it can be transferred to an object to be 
propelled. ${ }^{4}$ By integrating all of the segments through the kinetic chain, proper transfer and distribution of energy through joints was achieved, and this was demonstrated through an increase of velocity by those who performed functional training. The results between groups suggested that training to incorporate all the segments of the throwing motion and linking them together helps to add the force production produced proximally that can then be transferred through an efficient pathway. The fact that the experimental group's improvement from pre to posttest exceeded the MDC shows that this was starting to occur.

Though much power is derived from the core, throwing velocity also comes from minimizing energy leaks throughout the kinetic chain. ${ }^{30}$ Integration of proximal segments involving the stabilization of the trunk and scapula are key to proper arm function. ${ }^{31}$ Training the neural framework by working in functional patterns also allows for the proximal muscles to work in a stabilizing fashion, thus allowing the glenohumeral joint complex to both produce and distribute force to the ball. This was also seen in this study, as core and scapular stabilization exercises allowed for proper arm and scapula positioning, which is essential for upper limb function. ${ }^{28}$ The results of this study follow what was also shown by Saeterbakken et al, ${ }^{32}$ where a core stability program enhanced throwing velocity by $4.9 \%$. Proximal stability of the trunk and scapula help to create proper a proper throwing sequence, which can result in increased throwing velocity. The incorporation of force generated by the proximal segments via functional training program allowed for more force to be distributed to the ball. Furthermore, the force produced was better distributed to the ball by providing dynamic stability training throughout the kinetic chain. These benefits of the training program were shown by the moderate effect sizes through time as well as between group. Only the experimental group, however, improved well enough to 
exceed the MDC, showing that a functional training program produces clinically relevant improvements in throwing velocity.

\section{Closed Kinetic Chain Upper Extremity Stability Test}

Neuromuscular control is an important aspect of the throwing motion by relying on the body's efferent response to afferent signals, ${ }^{27}$ The efferent pathways that control the muscle actions must perform optimally in order to provide the arm with proper reflexive and stereotypical movement. ${ }^{24}$ The functional training program in this study intended to improve the neuromuscular function by providing exercises designed to stimulate and challenge glenohumeral stability. Specifically, closed kinetic chain exercises were used in order to stimulate mechanoreceptors within the glenohumeral joint. It has been reported that closed kinetic chain exercises stimulate mechanoreceptors in the glenohumeral joint complex, ${ }^{14}$ and elicit a neuromuscular response ${ }^{12}$ to dynamically stabilize the joint. The results of this study demonstrated that there was a large effect in the experimental group, showing improved neuromuscular control. However, the control group produced a moderate effect, indicating that neuromuscular control was not significantly influenced by the functional training program. These results are consistent with conflicting results found in the literature. One study found that closed kinetic chain exercises increased single-arm postural control, ${ }^{14}$ while another found no significant differences in single arm postural control for subjects performing closed kinetic chain exercises as compared to other training programs. ${ }^{15}$

Neuromuscular control is also influenced by preparatory movement. Proprioception from previous experiences are learned, stored, and used for planning and executing motor patterns, and combined with proprioceptive influences, will result in preparatory muscle activity. ${ }^{24}$ The functional training program in this study used motor patterns to allow the athlete to become 
aware of the joint's movements. As such, this information was used in conjunction with learned motor patterns to create preparatory muscle stiffness, which will in turn enhance neuromuscular control through dynamic exercise as seen by the large effect size through time by the experimental group.

All of the subjects in this study participated in practice and games during the course of this intervention. The increase in the control group's scores suggested that there was some neuromuscular control adaptations during the course of the season, as both groups' scores exceeded the MDC. The experimental group's changes illustrate that closed kinetic chain exercises used in conjunction with regular practice may produce a greater effect in neuromuscular control, but not enough to differentiate them from activity-matched individuals. Clinical Implications

The trends of the data are consistent with the direction of the hypotheses, most notably, throwing velocity, throwing accuracy, and neuromuscular control. These results indicate that the use of a functional training progression can result in clinically important outcomes with these variables. The functional training progression developed in this program can also be used in future studies. The subjects in this study progressed through the training program without difficulty in the exercises. The progression was not overly challenging to the patients and the exercises were simple enough for the subjects to complete successfully. This, combined with the fact that this program did not require the use of equipment, gives support to the use of functional training program within the clinical setting. Though there were few statistically significant results, throwing velocity and neuromuscular control improvements exceeded the MDC with only 12 sessions through six weeks, which helps to substantiate the use of a functional training program in strength and conditioning programs. 
The program used in this study may also be used in conjunction with general strengthening programs that may cause further improvements in performance from the players. Following a specific exercise progression that enhances the lower extremity, trunk, and upper extremity individually and then integrating them into one dynamic motion can produce clinically meaningful improvements in patients. This program can be used in off-season workouts for softball in order to improve performance measures such as throwing accuracy and throwing velocity that are important to the game of softball. Clinicians should take into consideration the use of the exercises and progression used in this study, as these results have demonstrated clinically meaningful improvements in this study. Moreover, this study also helps to demonstrate and support the use of functional exercise during rehabilitation and strength and conditioning activities. Overall, this study helps support the use of functional training within the clinical and strength and conditioning setting, and also indicates a new area of research that should be explored.

Limitations

There were several limitations in this study. First, the sample size was quite small, with only 19 participants completing the study in its entirety. Second, the training program was limited to only six weeks due to time constraints of this study. Furthermore, sessions from each week were limited to two, further marginalizing any training effect that may occur. The skill levels of the participants were also varied, and the margins of improvement for some subjects may be much smaller than the margins of improvement for others. The study also was hindered by other confounding variables. The fact that this study was performed during a softball season could have influenced the results. 


\section{CONCLUSION}

A functional training program for softball produced clinically-important outcomes. Throwing velocity, throwing accuracy, and neuromuscular control increased clinically by the MDC changes and effect sizes for those subjects participating in a functional training program. A functional training program will link the segments of the kinetic chain, producing important functional performance outcomes. Further research is needed to evaluate these results, and randomized controlled trials should focus on the effects of functional training programs both in softball and in other sports. 


\section{REFERENCES}

1. Hess, S. Functional stability of the glenohumeral joint. Manual Ther. 2000;5:63-71

2. Kibler W, Kuhn J, Wilk K, Sciascia A, Moore S, Laudner K, Ellenbecker T, Thigpen C, Uhl T. The disabled throwing shoulder: Spectrum of pathology -10 year updates. Arthroscopy. 2012;29:141-161

3. Fleisig G, Barrentine S, Escamilla R, Andrews J. Biomechanics of overhand throwing with implications for injuries. Sports Med. 1996;21:421-37

4. Sciascia A, Cromwell R. Kinetic chain rehabilitation: a theoretical framework. Rehabil Res Prac. 2012. 2012:1-9

5. McMullen J, Uhl T. A kinetic chain approach for shoulder rehabilitation. $J$ Athl Train. 2000;35:329-337

6. Drinkwater, Barbara. Women in Sport. Malden, MA: Blackwell Science, 2000. 626-646. Print.

7. Flyger N, Button C, Rishiraj N. The science of softball: Implications for performance and injury prevention. Sports Med. 2006;36:797-816

8. Oliver G. The windmill softball pitch: optimal mechanics and pathomechanics of injury. Athl Ther Today. 2010;15:28-31

9. Doyle F. Review of the windmill pitch: Biomechanics and injury. $J$ Chiropr Med. 2004;2:53-62

10. Glasser J, Caterisano A, Brown W. Off-season training for women's softball. Strength Cond J. 1999;21:54-59

11. Kritz M, Mamula R, Messey K, Hobbs M. In-season strength and conditioning programming for collegiate baseball pitchers: A unified approach. Strength Cond J. 2008;30:59-69

12. Prokopy M, Ingersoll C, Nordeschild E, Katch F, Gaesser G, Weltman A. Closed-kinetic chain upper body training improves throwing performance of NCAA division 1 softball players. J Strength Cond Res. 2008;22:1790-1798

13. DeRenne C, Ho K, Murphy J. Effects of general, special, and specific resistance training on throwing velocity in baseball: A brief review. J Strength Cond Res. 2001;15:148-156

14. Ubinger M, Prentice W, Guskiewicz K. Effect of closed kinetic chain training on neuromuscular control in the upper extremity. J Sport Rehabil. 1999;8:184-194 
15. Padua D, Guskiewicz K, Prentice W, Schneider R, Shields E. The effect of select shoulder exercises on strength, active angle reproduction, single arm balance, and functional performance. J Sport Rehabil. 2004;13:75-95

16. Lust K, Sandrey M, Bulger S, Wilder N. The effects of a six-week training programs on throwing accuracy, proprioception, and core endurance in baseball. J Sport Rehabil.2009;18:407-26

17. Carter A, Kaminski T, Douex A, Knight C, Richards J. Effects of high volume upper extremity plyometric training on throwing velocity and functional strength ratios of the shoulder rotators in collegiate baseball players. $J$ Strength Cond Res. 2007;21:208-215

18. Goldbeck T, Davies G. Test-retest reliability of the closed kinetic chain upper extremity stability test: a clinical field test. J Sport Rehabil. 2000;9:35-45.

19. Pezzullo D, Karas S, Irrgang J. Functional plyometric exercises for the throwing athlete. J Athl Train. 1995;30:22-6

20. Burkhart S, Morgan C, Kibler W. The diabled throwing shoulder: Spectrum of pathology part III: The Sick scapula, scapular dyskinesis, the kinetic chain, and rehabilitation. Arthrosopy. 2003;19:641-661

21. Borsa P, Lephart S, Kocher M, Lephart S. Functional assessment and rehabilitation of shoulder proprioception for glenohumeral instability. J Sport Rehabil. 1994;3:84-104

22. Palmer, Thomas. Effects of proximal stability training on sport performance and proximal stability measures. Diss. University of Kentucky, 2012. Print.

23. Crotin R, . Functional lower body strength and conditioning exercises for baseball pitchers: A current overview of lower body training for baseball pitchers. Perform Train J. 2011;10:8-12

24. Myers J, Lephart S. The role of the sensorimotor system in the athletic shoulder. $J$ Athl Train. 2000;25:351-363

25. Cohn J. Statistical Power Analysis for the Behavioral Sciences. $2^{\text {nd }}$ ed. Hillsdale NJ. Lawrence Erlbaum;1988

26. Hoch M, Mullineaux D, Andreatta R, English R, Medina-Mckeon J, Mattacola C, McKeon P. Effect of a 2-week joint mobilization intervention on single limb balance and ankle arthokinematics in those with chronic ankle instability. $J$ Sport Rehabil. 2014;23:18-26

27. Wilk K, Meister K, Andrews J. Current concepts in the rehabilitation of the overhead throwing athlete. Am J Sports Med. 2002;30:136-151 
28. Escamilla R, Andrews J. Shoulder muscle recruitment patterns and related biomechanics during upper extremity sports. Sports Med.2009;39:569-590

29. Horashima M, Kadota H, Sakurai S, Kudo K, Ohtsuki T. Sequential muscle activity and its functional role in the upper extremity and trunk during overarm throwing. J Sport Sci. 2002;20:301-310

30. Okada T, Huxel K, Nesser T. Relationship between core stability, functional movement, and performance. J Strength Cond Res. 2011;25(1):252-261

31. Mottram S. Dynamic stability of the scapula. Manual Ther. 1997;2:123-31

32. Saeterbakken A, Van Den Tillaar R, Seiler S. Effect of core stability training on throwing velocity in female handball players. J Strength Cond Res. 2011;25(3):712-718 


\section{APPENDICES}




\section{APPENDIX A}

\section{THE PROBLEM}

\section{Research Question}

Though not receiving as much attention as baseball, softball represents a sizeable portion of those athletes involved in overhead sports, with millions participating worldwide. ${ }^{7}$ Despite this, little research has been conducted in regard to scientific principles that underlay softball skills. Due to the close relationship between baseball and softball, it has been assumed that many of the skills in baseball are identical to those used in softball, especially in the overhead throwing motion. Many rehabilitation protocols and conditioning programs related to overhead throwing utilize exercises and concepts that are heavily based on research centered on baseball, with little evidence from studies that are specific to softball. This makes it increasingly difficult to examine current training methods for softball and to further develop an exercise program that is particular to softball alone. It is unclear what differences exist between the baseball overhead throw and the softball overhead throw, but it is clear that there is a lack of research specific to softball skills, due in part to the assumption that the two sports are too close in nature to warrant further research differentiating them. Nonetheless, it is still of great importance to examine softball in and of itself and how functional exercises affect performance. Proper conditioning and rehabilitation of softball injuries is no less important than those injuries seen in baseball. Focus on the biomechanics of overhead throwing, especially regarding rehabilitation strategies has been quite evident in the literature. Current concepts assert that functional and sport-specific exercises are key to the successful return of an athlete that has suffered an injury from overhead throwing 2, 19, 20, 33, 34 Improvements in neuromuscular control, strength, and muscle activation 
sequences are only a few of the proposed benefits of functional training. ${ }^{2,33,34}$ Yet as much as these exercises are lauded as essential to reinforcing proper throwing technique and biomechanical efficiency, there exists little evidence that examines the effect of a functional training regimen on throwing performance. There exists an even smaller body of research for a functional training program aimed at improving throwing performance in female softball athletes.

Though the body of evidence regarding functional training methods is limited and equivocal in nature, there have been a multitude of studies that have assessed the function of exact types of exercise to specific body parts. ${ }^{12,13,14,15,16,32}$ Though these studies have established the efficacy of one type of exercise over another in terms of performance, there remains a void of how to integrate these exercises into a comprehensive training. These studies examine exercises that are isolated to a single body segment, and rarely incorporate the entire kinetic chain. Furthermore, reasons as to why certain exercises improve performance over others remain unclear. Overhead throwing programs have utilized solely open kinetic chain exercises, closed kinetic chain exercises, or plyometric exercises, but have neglected the use of the core or the hips, even though they have been reported to be important in proper throwing motion. ${ }^{2,7,10,16,30,35,36}$ However, a full-body and sport-specific training program is not available for study, which begs the question as to what types of exercises closely relate to the overhand throwing motion of baseball.

Despite the fact that it is unclear which types (or combinations) of exercises most resemble the overhand throwing motion of baseball, the true aim of this or any of the studies is to examine the effect specific exercises have on performance. Studies have shown the comparative efficacy of one exercise over another, yet single plane motion is hardly functional during sport 
performance and only a single component to the mechanisms by which increased performance is achieved. To perform adequately, the entire kinetic chain must function properly to achieve the optimal result for the task at hand. ${ }^{3,5,16,33,37}$ When fatigue or failure occurs in a segment of this kinetic chain, decreased performance or even injury can ensue. ${ }^{16,35,37}$ Due to the lack of a comprehensive training program specific to overhand throwing, the answer to which exercises or combinations of exercises best improve throwing performance is further muddled.

There have been a variety of techniques to objectively assess the functional components of an extremity after a training program. From joint reposition sense to a pure strength assessment, current practice allows for a multitude of functional assessments to observe the effects of an intervention. These assessments are valuable to not only assess gains from an intervention, but to also show that the intervention had a specific and desired effect on a component of movement. Current concepts call for these functional assessments to be used as a resource to further approve or scrutinize a specific exercise or intervention, but it is also uncertain as to how these functional assessments relate to a specific task as well as to how these functional assessments can serve as a further measurement to predict performance.

Research Questions

1. What types of exercises (Open Kinetic Chain, Closed Kinetic Chain, Plyometric, a combination of all 3) are most sport specific to softball?

2. What types of exercises (OKC CKC, plyometric, combination) best improve throwing performance?

3. Will a functional training program improve functional assessments? (Joint Position Sense, single arm postural control)

4. Can these findings be extrapolated to other dynamic motions? (Batting performance, windmill pitch)

5. What rehabilitation strategies can best be put to use in performance training? 
Hypotheses

1. A functional training program for overhand throwing will significantly improve throwing accuracy compared to those who did not undergo the functional throwing training regimen.

2. A functional training program for overhand throwing will significantly improve throwing velocity compared to those who did not undergo the functional throwing training regimen.

3. There will be a significant improvement in joint reposition sense rotational error for those subjects who underwent the functional training regimen compared to those who did not.

4. There will be a significant improvement in joint reposition sense rotational error for those the right arm compared to the left arm in those who underwent the functional training regimen.

5. There will be a significant improvement in pre/post test scores for the closed kinetic chain upper extremity stability test for those who underwent the functional throwing training regimen compared to those who did not.

Assumptions

1. It is assumed that all subjects meet the inclusion criteria set forth by the primary investigator

2. It is assumed that all subjects undergoing testing will perform all maneuvers, exercises, and testing procedures to the best of their ability.

3. Subjects will be compliant and attend every training session assigned to them.

4. All instruments and equipment used in the study will be in proper working order and be reliable.

5. The primary investigator will be reliable in collecting results for throwing accuracy and throwing velocity.

\section{Deliminations}

1. The small sample size that will be used in this study is not generalizable to the entire softball population.

2. Participants in this study will be from a single Division III university.

3. All subjects will be between the ages of 18 and 23 


\section{Operational Definitions}

1. Anatomical Core - The lumbo-pelvic-hip complex consisting of 'local' and 'global' musculature that stabilizes spine and pelvis during activity. It is also responsible for the production and transfer of forces throughout the body. ${ }^{35,38}$

2. Closed Kinetic Chain - Exercise in which the terminal joint meets considerable resistance that prohibits or restrains its free motion. ${ }^{33}$

3. Concentric Contraction - Shortening of the muscle while it is being loaded. ${ }^{33}$

4. Core Stability - the ability to control the position and motion of the trunk over the pelvis to allow optimum production, transfer and control of force and motion to the terminal segment in integrated athletic activities. ${ }^{31,35,38}$

5. Dynamic Stability - Stability of the body during multi-planar functional movement. ${ }^{1,15}$

6. Eccentric Contraction - Elongation of the muscle while it is being loaded. ${ }^{33}$

7. Functional Exercises - Multi-planar, dynamic movements incorporating multiple joints that are similar to the movements used for a specific, real-life task. ${ }^{31}$

8. Glenohumeral Joint - The anatomical shoulder joint, made up of the bony articulation of the head of the humerus and the glenoid fossa of the scapula.

9. Glenohumeral Joint Complex - The combination of the scpaulothoracic joint and the glenohumeral joint.

10. Glenoid Labum - Ring of cartilaginous fiber surrounding the glenoid fossa that serves to provide further anatomical stability and nutrition for the joint.

11. Joint Reposition Sense (JPS) - An individual's ability to consciously recognize where their joint is in space. ${ }^{24}$

12. Kinetic Chain - The integrated, multisegmented, sequential joint motion and muscle activation system. ${ }^{4}$

13. Neuromuscular Control - The unconscious ability to activate dynamic stabilizers occurring in response to joint movement for the purpose of maintaining functional joint stability. $^{24}$

14. Open Kinetic Chain - Exercise in which the terminal joint is free to move. ${ }^{33}$

15. Performance - The execution of a task for a specific aim or purpose without direction or guidance. 
16. Plyometric - Powerful muscle contractions after rapid stretching or dynamic loading of the same muscle group. ${ }^{19}$

17. Rotator Cuff - The four muscles that provide dynamic stabilization of the shoulder joint. These muscles are the infraspinatus, the supraspinatus, the teres minor, and the subscapularis.

18. Scapulothoracic Joint - The functional joint consisting of the movement of the scapula moves along the thoracic spine and posterior ribcage. ${ }^{2}$

19. Session - One bout of planned and controlled exercise.

20. Sport Specific Exercises- Movements mimicking those movements that may be used in a sport i.e. overhand throwing in baseball, kicking in soccer, etc.

Limitations

1. Subjects may be injured and drop out of the study

2. Subjects may leave the team and drop out of the study

3. Subjects may drop out of the study by choice

Significance of the Study

The enhancement of performance and the means by which it can be attained is a high priority in today's sports world. Through almost all of athletes, strength and conditioning programs receive great attention, and a multitude of research has been dedicated to help improve ways by which we can run faster, hit harder, throw farther, and perform better. With this in mind, research should not only study the mechanisms by which the body functions, but also how these mechanism can work in conjunction to produce remarkable results. Current research is lacking regarding the effects of a comprehensive work out program and its effect on throwing performance, especially in regards to softball athletes. Not only will it detail how a sportspecific functional program impacts throwing performance, but also to help validate the current concept of functional exercise being instrumental in strength and conditioning and rehabilitation programs alike. Furthermore, it also aims to explore a relationship between functional 
assessments and how they are related to actual performance. Functional progressions are common in rehabilitation, but their use in strength training programs are spotty and lacking in validity. This study will provide only a step in assessing how functional progression should be used in training regimens for overhand throwing in college softball athletes. Furthermore, the results of this study will guide future research on the ways that softball skills can be improved. Exercises and progressions used in this study could be used in further research, in whole or in part. The results of this study can be disseminated through publication or workshops aimed toward further understanding of throwing progressions and training programs. 


\section{APPENDIX B}

\section{LITERATURE REVIEW}

Introduction

The body of evidence pertaining to functional training programs is small, especially how functional training relates to softball. Though softball has increased in popularity significantly over the course of the last few decades, ${ }^{6}$ little evidence has come out that is specific to the skills used in softball, especially the overhead throwing motion. In 1970, less than 10,000 girls were playing softball in high schools. ${ }^{6}$ Today, the number of youth softball players is said to be over $349,000 .{ }^{9}$ Recreationally, it is also estimated that over 40 million people participate, making it the most popular recreational sport in the US. ${ }^{6}$ The International Softball federation currently lists 122 national federations as members. ${ }^{7}$ Despite the growing popularity of the sport, there is minimal evidence related to softball training and the mechanics that underlay the protocols. Sport-specific training techniques is often de-emphasized or altogether unknown within the sport, ${ }^{10}$ which could be a contributing factor as to why there is a higher injury rate for girls in softball than boys in baseball. ${ }^{8}$ Due to the similarity between softball and baseball, both strength and conditioning programs and rehabilitation programs are based on research and biomechanical principles developed through baseball research. Overhead throwing in softball is commonly thought as identical and analogous to the overhead throwing motion in baseball. ${ }^{6}$

The throwing motion is a highly complex motion that involves a coordinated and sequential action of the entire body to be completed. Deficits in range of motion, strength, and proprioception have all been implicated in a wide variety of injuries. ${ }^{2,27}$ It is known that the throwing motion is a dynamic activity that incorporates multiple segments through all three 
planes of the body. ${ }^{2}$ All segments of the throwing motion serve a specific purpose and work synergistically with each other to perform dynamic movement., and is aptly called the 'kinetic chain'. As such, strength training, conditioning, and rehabilitation protocols should follow this principle, but most do not. There has been a plethora of literature ${ }^{1,2,3,7,13,19}$ pertaining to the biomechanics of the throwing motion and the phases therein, and these principles have guided glenohumeral joint complex rehabilitation programs. Many principles of these rehabilitation programs have been used in strength and conditioning techniques as well. However, many programs isolate the segments and work on the individual performance characteristics. Integration of the upper extremity, core, and lower extremity should be used when attempting to enhance the throwing motion. ${ }^{4,5}$ Some exercises have come from evidence-based research, but many also come from best clinical practice. It is important to consider the kinetic chain when developing sport-specific training programs, but no functional progressions specific to softball have yet to appear in the literature. This literature review will analyze the body of research out there pertaining to the sport of softball, the throwing motion, pathophysiology of the throwing motion, the segments of the throwing motion including the glenohumeral joint complex, core, and lower extremity, and current training techniques.

Anatomical Roles of the Glenuhumeral Joint Complex, Core, Lower Extremity, and Kinetic Chain

The throwing motion is a full-body dynamic activity involving multiple segments of the body. Each of these segments is involved in basic anatomical roles, including stabilization and producing motion. During dynamic activity, these roles change, and the segments interact with each other through what is known as the kinetic chain. The segments included in the overhead throwing motion include the glenohumeral joint complex, the core, and the lower extremity. 
Anatomical roles of the glenohumeral joint complex: The glenohumeral joint complex is one of the most complex joints in the body, with mobility and stability constantly in a delicate balance throughout functional tasks. The glenohumeral joint complex is made up of four joints: the sternoclavicular joint, the glenohumeral joint, the acriomioclavicular joint, and the scapulothoracic joint. The only true joint that connects the glenohumeral joint complex to the body is the sternoclavicular joint, which articulates the proximal end of the clavicle to the superior aspect of the sternum. The sternoclavicular joint and the acriomioclavicular joint are not thought to have much function or effect on throwing, as it is a syndesmosis and has limited motion to it. However, there are arthorokinetmatic motions that must occur to allow for clavicular elevation and rotation that occur concomitantly with scapular upward rotation. In dynamic motion, the glenohumeral joint and scapulothoracic joint are of primary concern.

The glenohumeral joint is what is what is commonly referred to as the true shoulder joint, where the head of the humerus articulates with the glenoid fossa of the scapula as a ball and socket joint. This joint is inherently unstable due to relatively shallow bony geometry of the glenoid fossa. There are, however, complementing and stabilizing features of this joint that allow for the glenohumeral joint complex to perform effectively, even during intensive athletic tasks. The glenoid labrum is a cartilaginous ring of tissue that serves to deepen the fossa and allow for more static stability of the joint. Current thinking, however, has evolved, and the labrum may have a more functional role. The labrum is a highly deformable structure that can more evenly distribute contact pressures and lubrication, which can maximize proper movement and concavity compression characteristics. ${ }^{2}$ Moreover, it can act as a pressure sensor that can send proprioceptive feedback. Lastly, it serves as an attachment site for ligaments and muscles which can optimize the tension. ${ }^{2}$ The glenohumeral ligaments also play an important role in the 
glenohumeral joint complex to provide static stability as a 'passive' system of stability. ${ }^{1}$ The joint capsule provides little stability in and of itself, as it has been reported that it allows for nearly $2 \mathrm{~cm}$ of humeral translation. ${ }^{1}$ The superior glenohumeral ligament and coracohumeral ligament prevent inferior displacement and limit external rotation between 0 and 60 degrees of abduction. The middle glenohumeral ligament provides stabilization for anterior translation of the humeral head and also limits external roation from 0 to 90 degrees of abduction. Finally, the inferior glenohumeral ligament limits posterior translation of the head of the humerus during elevation and internal rotation. ${ }^{1}$ These ligaments also provide proprioceptive feedback that helps position the joint correctly during dynamic activity. The muscles of the glenohumeral joint complex also provide specific and important roles. The supraspinatus, infraspinatus, subscapularis, and teres minor are collectively called the 'rotator cuff', and are highly responsible for the dynamic stability of the glenohumeral joint complex. The infraspinatus and teres minor externally rotate the humerus. The supraspinatus helps initiate arm abduction along with the deltoid, and the subscapularis internally rotates the humerus. Primarily, however, the rotator cuff serves to stabilize the head of the humerus in the glenoid by co-contraction of the muscles. Based on the insertions and line of force produced by the muscles, the head of the humerus will articulate and compress into the glenoid fossa. ${ }^{1}$ This action occurs with nearly all dynamic movement. Other muscles such as the latissimus dorsi, pectoralis major, long head of the biceps brachii, and deltoid also play a role in maintaining stability while causing movement. ${ }^{1}$

The scapulothoracic joint is the third joint that makes up the glenohumeral joint complex. It is not a true joint, with no direct articulation between bone surfaces. It serves as a functional joint, and the scapula's motion along the posterior ribcage is the point of analysis. ${ }^{31}$ The scapula serves as a base and origination of the rotator cuff musculature, and as such optimizes the length- 
tension relationship of these muscles. ${ }^{31}$ Moreover, its movement complements that of the glenohumeral joint, and increases stability of the joint by its own motion. ${ }^{2}$ The fact that the scapulothoracic joint is not a true articulation of bones means that there is a lack of ligamentous stability. As such, the muscles surrounding the scapula that originate on the thorax have recruitment, proprioceptive, and contractile properties. ${ }^{31}$ These muscles include the trapezius, rhomboid major, rhomboid minor, serratus anterior. In the same way that the rotator cuff functions, these muscles will co-contract to produce stability of the scapula, ${ }^{31}$ but can also contract synergistically to create dynamic movement in the glenohumeral joint complex, such as overhead throwing. ${ }^{2}$

Anatomical roles of the core: Traditionally, the core has been described as consisting of the structures that encompass the trunk, specifically the lumbo-pelvic region. ${ }^{30,35,38,39}$ The core can and has been described as consisting of three basic parts that all provide unique contributions to core stability and function. ${ }^{22,37}$ The first part is a passive subsystem that provides static support, and includes the facet joints, intervertebral discs, ligaments, and joint capsules. Ideally, this subsystem only operates during the extreme ends of the range of motion, as it has been reported that the passive subsystem of the spine will become overloaded during pressures of 2-9 $\mathrm{kg}{ }^{38}$ The second subsystem of the core is an active musculoskeletal system that either generates force by isotonic contraction or transfers force via isometric contraction. The muscles of the abdomen, including the Transverse Abdominus, the Internal Obliques, External Obliques, Rectus Abdominus, Erector Spinae Musculature, Multifidus, Quadratus Lumborum and Pelvic Floor muscles are all the customary muscles of this subsystem. As mentioned before, consistency regarding what muscles are included in the core is lacking, and muscles of the hips such as the Gluteal muscles and Iliopsoas have been reported to be involved in the anatomical core. ${ }^{37}$ It has 
also been suggested that the Diaphragm is also involved in core stability by increasing intraabdominal pressure. ${ }^{38}$ The musculature of the core has also been divided further into two basic groups, aptly dubbed 'local' muscles and 'global' muscles. ${ }^{23,35}$ The local musculature refers to those muscles that have attachments to the lumbar vertebrae themselves and are primarily tasked with inter-segmental vertebral stability. ${ }^{35}$ Examples of local muscles might include the Multifidus and the Transerse Abdominus. Although the Transferse Abdominus does not attach to the spine directly, it is an integral part of local musculature in that it is active only in spinal stabilization and not force generation. ${ }^{35}$ Conversely, the global muscles are those muscles that have attachments outside of the core, either to the hips and pelvis or to other regions of the spine. Primarily, the muscles are responsible for force generation to either direct spinal orientation or provide resistance to external forces. Examples of these would include the Erector Spinae, Rectus Abdominus, Internal Oblique, and External Oblique. Core stability is best achieved by this subsystem providing the majority of the core stability. The final proposed subsystem is that of the sensorimotor neural feedback system that will instruct and/or modulate the active musculoskeletal system, depending on the desired movement or in response to outside perturbations. It is important that all three subsystems work harmoniously with each other to allow proper athletic movements. ${ }^{40}$

The role of the core during athletic activities has been questioned, often due to a gross lack of meaningful data and evidence surrounding the role of each muscle group and the synergistic relationships. It is known that the function of any single core muscle will vary between dynamic movements. ${ }^{41}$ Together, the core has the ability to produce motion in all three cardinal planes. Due to anatomical origins and insertions, many muscles have the capability of producing more than one type of motion. Though the mechanisms are poorly understood, 
several factors must be considered in the function of the core: First, each core muscle can only directly produce force and provide stability in the direction of muscle fibers; second, the mechanical properties of all core muscles are affected by the posture of the individual and are subject to the force-velocity and force-length relationships; and third every activity will cause stress to the system as a whole because of the differences in the magnitude and direction of internal and external forces. It is believed that because of these principles, core stability is more heavily reliant on the co-contraction and synergistic relationship of the muscle group as a whole as opposed to the individual stability that one muscle provides. ${ }^{37,38,41}$ In regards to functional movement, the core can be described as the central anatomical base for movement in the extremities, and the concept of 'proximal stability for distal mobility' has been a central theme in the analysis and development of core strengthening programs. ${ }^{38}$ It has been reported by some researchers that all movements either originate or are coupled through the trunk. ${ }^{16,30,41,42}$ This apparent anticipatory initiation of movement in the trunk as a whole has yet to be shown to occur in all tasks thus questioning the need for specialized training. ${ }^{31}$

Anatomical roles of the lower extremity: The lower extremity serves as a base for many movements and dynamic activities. As the weight bearing segments of the body, they are invariably a vital component of any dynamic movement. The stabilization and proprioception actions of the lower extremity provide for a stable base of dynamic activities, especially those incorporating the upper extremity. The pelvic muscles, including the gluteals, work in conjunction with the trunk musculature to fascilitate the initiation and transfer of forces. ${ }^{35,41,42}$ Furthermore, they serve to stabilize and dissipate ground reaction forces in order to prevent injury and prevent compensation by the upper extremity. ${ }^{23}$ 
Anatomical role of the kinetic chain: The kinetic chain can be described as a system that links the proximal and distal segments of the body to perform fundamental movement. ${ }^{22}$ Movement, especially athletic functional movement, involves a multitude of different forces that are distributed through all three planes of the body. The synergistic relationship of the body segments via this kinetic chain is vital in order to execute efficient and safe movement. The goal of many athletic training activities such as overhead throwing is to impart a large amount of force to the distal segment, ${ }^{5}$ and to do this a sequential coordination of proximal to distal activation must occur. Normal motor patterns of upper extremity movement are characterized by lower extremity and trunk musculature before activation of the arm. ${ }^{2,33}$ This linkage allows for efficient and effective as well as safe transfer of energy and momentum from the proximal segments to the distal segments. ${ }^{5}$ Functional tasks are highly dependent on the kinetic chain appropriately working as a single unit, ${ }^{4}$ and the pattern of proximal muscle activation before distal muscle activation serves as a foundation to drive energy through the system, especially the scapula and the glenohumeral joint. ${ }^{5}$ Moreover, the proper transfer of energy through the kinetic chain mitigates the loads received in the glenohumeral joint complex that would be seen without the full-body motion, marginalizing the needed contribution of the glenohumeral joint complex ${ }^{5}$ and lowering the risk of injury.

The synergistic relationship between the musculature involved in a given movement pattern employs a complex and refined pattern of both feed forward and feedback loops. Anticipatory actions from the leg and trunk are commonly seen. Local core musculature activation will precede athletic function, and are associated with distal joint movements, ${ }^{37}$ especially during voluntary arm movement. ${ }^{5}$ Disturbances in balance equilibrium are also 
counteracted by these proximal-distal synergies. ${ }^{5}$ Postural adjustments are sensed from all segments of the body, and specific stabilization movement is seen throughout the kinetic chain.

According to Kibler et al, $^{2}$ the kinetic chain has several functions. First, it facilitates muscle activation patterns that link multiple segments into one functional segment, thereby decreasing the degrees of freedom within the movement. Second, it provides a stable proximal base for distal mobility. Third, it maximizes the forces created by larger proximal muscles and transfers it to the hand. Fourth, it produces interactive movements at the distal joints that create more force than they would be able to create individually. Fifth, it produces torques that decrease deceleration forces. ${ }^{2}$ Overall, the kinetic chain provides the ability for proximal and distal segments to interact to create joint stiffness or rotations to perform effective dynamic movement. ${ }^{16,22}$ Pathophysiology

Overhead throwing is a skill that involves a plethora of forces that have a high magnitude throughout the joints of the upper extremity. It is a skill that involves almost every joint of the body and thus, involves specific coordination and organization of these segments to perform an extreme yet precise skill. ${ }^{2}$ Given this fact, there are several underlying deficiencies that may predispose a thrower to injury.

Glenohumeral rotational deficits is one factor that has been commonly implicated in pathological glenohumeral joint complexs. ${ }^{2}$ Throwers will tend to have deficits in internal rotation along with combined excessive external rotation in the throwing glenohumeral joint complex. Though this can be construed as pathologic, it has been shown that the total range of motion (TROMD) of the glenohumeral joint complex is the same as the contralateral glenohumeral joint complex. This range of motion should not exceed 186 degrees, however, and 
Kibler and colleagues ${ }^{2}$ suggested that the asymmetry between glenohumeral joint complexs greater than 5 degrees puts the thrower at an increased risk of injury. Glenohumeral internal rotation deficits (GIRD) also has implications for injury. It was reported that a prospective study demonstrated that a GIRD of 18 degrees puts a thrower at 1.9 times increased risk of injury. ${ }^{2}$ The rotational deficits do not cause injury by themselves, but only alter glenohumeral joint complex biomechanics and place abnormal loads on other supportive structures of the glenohumeral joint complex. These rotational changes are the results of repetitive throwing, changing the anatomical joint geometry, muscular length and contractile ability, and capsuloligamentous features. Bony remodeling occurs on the scapular side of the dominant arm with increased glenoid retroversion as a result of the shear forces and torsions placed upon the glenohumeral joint. ${ }^{2}$ Muscular changes include thixotropy, which limits the contractile properties of muscles and increases stiffness. Furthermore, chronic strain on musculature can cause the muscle itself to shorten. ${ }^{2}$ The anterior glenohumeral ligament has been shown to lengthen while the inferior ligaments have been shown to be thickened on patient imaging. ${ }^{2}$ Altered range of motion in the glenohumeral joint complex can change the length-tension relationships of the musculature and also cause altered kinematics that may be improper and lead to impingement or muscle strains. Increased external rotation is thought to cause partial-thickness tear of the rotator cuff through compressive loads and internal impingement. ${ }^{2}$

Even though a thrower's glenohumeral joint complex may be anatomically different, it does not necessarily mean it is pathologic. Anatomical changes have been consistently seen in asymptomatic thrower's glenohumeral joint complex that would otherwise be described as pathologic. This suggests that the anatomical changes may be adaptive in nature rather than pathologic. Increased anterior glenohumeral joint complex laxity and partial thickness rotator 
cuff tears could occur in order to be able to perform the extremes of high-velocity throwing. ${ }^{2}$ Clincal examination and rehabilitation should focus on scapular dyskinesis, TROMD, GIRD, and kinetic chain deficits. $^{2}$

Scapular strengthening is another component of many rehabilitation efforts, as the movement of the scapula directly influences the motion of the glenohumeral joint and thus, the throwing motion. The glenoid must be positioned properly and congruously in order to maintain contact with the head of the humerous as it moves through high velocity rotation. It must also smoothly retract and protract as it moves from the wind up phase to the follow through phase. Thirdly, it serves as a base for the rotator cuff musculature, and as such needs to properly move in order to maintain proper activity of these muscles to promote glenohumeral stabilization. ${ }^{34}$ Altered scapular mechanics have been implicated in many pathologies, including labral tears, tendonitis, and rotator cuff strains. 2,20

Neuromuscular control represents another component that is vital to the throwing motion. Feed-forward mechanisms prepare the glenohumeral joint complex for dynamic movement and allow for dynamic stability from the surrounding musculature during functional movement. ${ }^{24}$ The lack of neuromuscular control, however, leaves the glenohumeral joint complex prone to injury. A lack of awareness of the joint in space leads to improper muscle activation, which can lead to faulty mechanics. Proprioception arises from sensory inputs from the skin, joint tissues including capsular and ligamentous structues, and muscles. ${ }^{24,43}$ Consciously, proprioception allows for proper distal extremity placement while unconscious proprioception modulates muscle function. ${ }^{21}$ In the context of overhand throwing, it is vital to both prevent injury as well as to improve performance. Proper hand placement can help mitigate the amount of torque put on the shoulder as well allow for proper transfer of forces distally by conserving momentum and 
maximizing angular velocity and force that can be transferred to the ball. Moreover, improving joint position sense enables joint stability, resulting in appropriate muscle action for smooth, biomechanically sound motion. ${ }^{1}$ The elicitation of input signals during the overhand throwing motion can cause reflexive feedback mechanisms that stimulate muscle contraction, thereby providing dynamic stabilization within the shoulder joint. ${ }^{24}$ If muscle coordination is impaired due to deficits in joint position sense, symptomatic shoulder instability can occur. ${ }^{44}$ Repetitive throwing may desensitize the muscle spindles, golgi tendon organs, and other mechanoreceptors responsible for neuromuscular control, and may put the glenohumeral joint complex at risk. ${ }^{24} \mathrm{It}$ has been shown that joint proprioceptors do not recover as quickly as the muscle itself. ${ }^{45}$ Muscle fatigue hinders proprioception, and joint position sense suffers, again leading putting the glenohumeral joint complex at risk.

The Throwing Motion

The overhead throwing motion has been studied extensively through the years. Traditionally, the overhead motion has been described as a more unnatural motion of the glenohumeral joint complex joint, and injuries due to the motion are well-known. ${ }^{3}$ Proper biomechanics of throwing are of great importance to both improve performance and reduce the incidence of injury.

The overhead throwing motion is divided into six distinct phases: Wind up, stride, arm cocking, arm acceleration, arm deceleration, and follow through. ${ }^{3}$ Each phase has specific biomechanical principles that allow a thrower to channel forces safely and effectively during the overhead throwing motion. ${ }^{3}$ The body of literature will have different titles for the phases, but the phases themselves remain consistent throughout current research. 
During the wind-up phase, the thrower will stand perpendicular to the target, with the non-throwing hand serving as the 'lead' side. The thrower will then shift the weight to the back leg and bring the hand with the ball to the chest. The lead leg is taken off the ground, and the stance leg is slightly bent. The purpose of this step is to put the thrower in a proper position to correctly move through the rest of the throwing progression. ${ }^{46}$

The next step of this process is the stride phase. This phase is to begin the generation of linear forces toward the target. The phase begins when the lead leg begins to move toward the target, initiated by stance leg hip abduction followed by hip extension and knee extension. Concurrently, the stance leg will internally rotate and the lead leg will externally rotate. This momentum shift in the lower extremity will begin the force generation needed to propel the ball forward. During this phase, the thrower will also abduct the arms and stretch the anterior musculature, therefore storing energy in the elastic components of the muscles. This will also enhance the stretch reflex, which will enhance voluntary muscle contraction for the following throwing phases. The deltoid and supraspinatus are primarily responsible for the abduction of the throwing glenohumeral joint complex, while the infraspinatus, subscapularis, and teres minor maintain glenohumeral positioning. ${ }^{3,28}$ The upper trapezius and serratus anterior upwardly rotate the scapula for further glenohumeral stability and positioning. ${ }^{28}$ The wrist and fingers move into extension during this phase for proper grip and positioning of the ball in the hand. ${ }^{3}$ The phase ends when the lead leg comes in contact with the ground. At the end of this phase, glenohumeral joint complex is at 90 degrees abduction and externally rotated. The elbow is flexed and the wrist is in extension. The trunk remains perpendicular to the target.

The third phase of the throwing is arm cocking, where the body gets into a position to throw. This phase starts from the lead leg being planted to maximum glenohumeral external 
rotation of the throwing glenohumeral joint complex. The lead leg contracts concentrically to slow knee flexion and then isometrically to stabilize the lead leg. The hip, trunk, and upper torso begin to rotate towards the target as well as hyperextend in preparation for more force production. Throughout this phase, the trunk remains perpendicular to the ground. The angular velocity of the hips has been shown to be from $600-750$ degrees per second ${ }^{46}$ while the upper torso angular velocity has been measured at approximately 1100-1300 degrees per second. ${ }^{46}$ The rotation of the musculature around the pelvis and torso generate a large amount of force that needs to be transferred to the throwing arm. In the overhead motion, it has been shown that a $20 \%$ decrease of the energy produced by the trunk results in a requirement of $34 \%$ more arm velocity required to deliver the same amount of energy to the ball. Inadequate bend in the knees that breaks the kinetic chain results in $23-37 \%$ increased loads in the glenohumeral joint complex. $^{38}$

In the glenohumeral joint complex, the levator scapulae, serratus anterior, trapezius, and rhomboids work to stabilize the scapula in order to promote safe, and more importantly efficient glenohumeral action. ${ }^{2,3,28,31}$ The glenohumeral joint remains in 90 degrees of abduction while it externally rotates from between $150-180$ degrees. ${ }^{3,4}$ During this time, the rotator cuff muscles are very active in order to resist glenohumeral instability. The pectoralis major, latissimus dorsi, subscapularis, and anterior deltoid produce an internal rotation torque to resist anterior translation of the head of the humerus during maximal external rotation. This torque has been reported to be from $65-70 \mathrm{~N}-\mathrm{m}^{6}$ along with $350-610 \mathrm{~N}$ of compressive force. ${ }^{3}$ At the elbow, a large valgus force is produced due to the increased external rotation combined with the rotation of the proximal segments. This has been measured to be 240 to $420 \mathrm{~N}$ of medial force at the elbow joint. Maximum elbow flexion is achieved at 100 degrees. Before the end of this phase, 
the elbow will start to extend, however. This is due to the fact that at a reduced flexion angle at the elbow reduces the moment of inertia during external rotation, allowing for greater angular velocity at the glenohumeral joint complex. ${ }^{46}$

Following the arm cocking phase, arm acceleration occurs that serves to both generate and transfer forces to perform a throw. It occurs between maximal external rotation and the point of release. The hips and trunk continue to rotate towards the target but also begin to flex to a neutral position at release. Trunk flexion occurs primarily from the internal and external obliques as well as the rectus abdominus. The lead leg continues to act isometrically or even straightens slightly isotonically to continue to provide a stable base of support. ${ }^{3}$ The glenohumeral joint complex continues to be in a 90 degree abducted position, but the glenohumeral internal rotators act concentrically to produce high internal rotation angular velocity, measured at approximately 7000 degrees per second. ${ }^{28}$ Maximal internal rotation occurs at or near ball release. These muscles include the subscapularis, pectoralis major, and teres major. The scapular stabilizing muscles are also highly active, further promoting scapular control and stabilization at this phase. ${ }^{3}$ The elbow continues to extend to further mitigate the arms moment of inertia, thus promoting internal rotation angular velocity. Maximum elbow extension occurs in the middle of the acceleration phase. There are conflicting reports as to whether the elbow extension is a result of the posterior musculature around the elbow or as a result of centrifugal forces causing elbow extension. It is believed that kinetic energy transferred to the arm from the lower extremity and trunk produce elbow extension, causing an angular velocity of approximately 2300 degrees per second. ${ }^{46}$ The wrist moves from a hyperextended position to a neutral position, and is the final segment to impart force on the ball. Wrist flexors are also highly active in this phase, indicating that they concentrically contract in order to further generate force. ${ }^{3}$ 
After the body generates the power necessary for arm acceleration, the arm must be decelerated. This phase is generally short, lasting only until maximal glenohumeral joint complex internal rotation, which is generally until a 0 degree neutral position is reached. ${ }^{46}$ The glenohumeral joint complex will also horizontally adduct to increase deceleration forces. During this time, posterior and horizontal abduction torques are produced by the latissimus dorsi, teres major, and posterior deltoid. Large compressive forces at the glenohumeral joint complex and elbow also occur at this phase in order to prevent distraction of the joint. These forces have been measured at $980-1200 \mathrm{~N}$ and $800-100$, respectively. ${ }^{46}$ There is high activity of the scapular stabilizing musculature that is meant to resist scapular protraction. ${ }^{28}$ The elbow flexors and wrist extensors work eccentrically to slow elbow extension and wrist flexion as well.

The final phase is the follow through, which starts at maximum internal rotation and ends when the arm has finished its movement across the body and when the thrower is in a balanced position. The flexing of the trunk and extension of the lead knee allow energy from the throw to be dispersed into the larger muscles of the legs as well as the ground. ${ }^{3}$ In the glenohumeral joint complex, the follow through is much the same as the deceleration phase, with the exception that the compressive forces in the glenohumeral joint complex and elbow are generally less than the forces in the deceleration phase. Furthermore, the rhomboids and middle trapezius act eccentrically to control scapular protraction. ${ }^{3}$

Role of the glenohumeral joint complex in the throwing motion: In the throwing motion, the glenohumeral joint complex is responsible for a large part of the motion, and is the key for both the production and distribution of forces produced by proximal segments. The inherent mobility of the glenohumeral joint complex allows for a great degree of freedom and function, 
and can thus allow for high velocity ballistic movement that can be complemented by full-body force production.

The surrounding musculatures of the glenohumeral and scapulothoracic joints are important for throwing. The muscles of the glenohumeral joint first serve to provide compressive forces as well as shear forces that counteract those forces that occur as a result of high velocity movement, ${ }^{1,3}$ while also providing proprioceptive feedback for the body in order to regulate forces and muscle co-contraction. In order for normal movement to occur, this proprioceptive feedback system must function properly. ${ }^{1,24}$ Stabilization and coordination of the joint architecture is necessary for proper functioning throughout the entire motion. The scapular stabilizing muscles serve these functions as well, dynamically contracting to stabilize the scapula as the arm moves through the overhead throwing motion. There is a pattern of progressive retraction, upward rotation, and controlled axial rotation that allow the arm to be stabilized throughout the motion. ${ }^{2}$ The musculature of the glenohumeral joint complex also serves to produce force and direct forces during the throwing motion. During the arm cocking phase, the stretching of the anterior musculature creates a multitude of potential energy that is used for the forward ballistic movement. ${ }^{47}$ The glenohumeral joint complex also inherits and distributes the forces produced by the hips and trunk and directs it to the most distal joints of the arm. As a part of the kinetic chain in the throwing motion, the glenohumeral joint complex will invariably receive energy and force from the proximal segments, modulate and direct it toward the distal segments. ${ }^{2}$

Role of the core in the throwing motion: The analysis of upper body motion, specifically overhead movement patterns, torque production seems to be the primary role of the core. ${ }^{41}$ During pitching motion, it has been reported that the activation of the Transverse Abdominus 
precedes that of muscles in the arm as much as $30 \mathrm{~ms},{ }^{30,37,41}$ and a $75-100 \%$ increase in the activation level of the contralateral oblique as compared to static stability exercises. ${ }^{30,41} \mathrm{~A}$ general pattern of force development from the ground up has been consistently shown. ${ }^{38}$ Continuing with this pattern, almost $85 \%$ of the deceleration force in a forward moving arm during pitching comes from the periscapular and trunk musculature rather than the rotator cuff. ${ }^{38}$ In terms of performance enhancement, there has been little evidence to conclusively say that core stability and strengthening programs effectively enhance an athlete's ability to bat, pitch, or hit. ${ }^{35}$ In the analysis of the change in throwing velocity after a core stability intervention, one study provided evidence that throwing velocity did in fact increase $4.9 \%$, although the population size was small. ${ }^{32}$ This supports a previously reported statistic that the hip and trunk contribute $50 \%$ of the kinetic energy to the entire throwing motion. ${ }^{38}$ Although the changes in throwing velocity can be attributed to an increase in core strength, it is unclear by what mechanism this occurred. ${ }^{32}$ Though throwing velocity is an important measure of performance, accuracy is also important. In another study, athletes from a Division-III collegiate baseball team underwent core training and then were subjected to throwing testing that was measured by the Functional ThrowingPerformance Index. ${ }^{16}$ What was found was that there weren't any differences between the experimental group and the control group in terms of the core stability as measured by the Sorenson back extensor test and the abdominal fatigue test. ${ }^{16}$ There were also no significant increases in throwing accuracy, either, which further confounds the efficacy of core stability exercises. $^{16}$

Role of the lower extremity in the throwing motion: Though the lower extremity is not traditionally thought of as an integral part of the throwing motion, it is in fact vital to producing an effective and safe throw. The hip musculature functions to start force production and also to 
stabilize the body. It works in conjunction with the core musculature to allow for both the transfer and production of forces and also to stabilize the body in order to allow these forces to be transferred properly through the distal segments. ${ }^{35,38}$ The lower extremities are responsible for initiating drive forces, bracing forces, and the transfer of momentum through the kinetic chain. ${ }^{23}$ Weakness of hip abductors or inflexibility of non-dominant hip rotation causes breakages in the kinetic chain. This will increase lumbar lordosis in acceleration, causing the arm to be placed behind the body and creating a hyperabducted/external rotation moment. ${ }^{20}$ Proper activation of these muscles in sequential order with other musculature is necessary for the throwing motion, as a proximal-to-distal and ground-up pattern has been consistently observed. $28,29,38$

The role of the lower extremity in the overhead throwing motion is a relatively new topic in the literature, and represents an emerging topic of interest in research. ${ }^{23}$ Despite this there is very little research regarding specific roles of the lower extremity has yet to be explored. Core literature include the lower extremity and hip musculature as part of the core, and as such work synergistically with the musculature of the trunk to perform dynamic movement. ${ }^{35,38}$

Role of the kinetic chain in the throwing motion: Dynamic activity like overhead throwing is a complicated skill involving multiple segments moving in a coordinated fashion throughout all three planes of the body. As such, it is important that each work in conjunction with the other in order to provide a safe and effective motion.

Only four body motions contribute to ball velocity: stance leg hip abduction, trunk rotation, glenohumeral joint complex internal rotation, and elbow extension. ${ }^{2}$ It must be noted, however, that the positive contribution of elbow extension is only from interaction with the motions of the glenohumeral joint complex and trunk rotation, reinforcing the fact of how the 
proximal segments' force production are transferred to the distal segments. ${ }^{2}$ Continuing with this, increases in velocity of a baseball throw are most correlated with increases with trunk rotation. ${ }^{2}$ Also, maximum ball velocity is correlated strongly with the number of segments being used, with the slowest ball velocity measured with arm use only and the fastest with a throw integrating the legs and hips. ${ }^{2}$ It has been reported that the trunk/hips contribute to around $50 \%$ of the force produced in the entire throwing motion, ${ }^{38}$ but others report it as low as $46 \% .{ }^{13}$ Muscle activation patterns of a baseball throw shows sequential activation of the contralateral hip to the posterior trunk and finally up to the glenohumeral joint complex. ${ }^{2,3,28,29,35}$ The throwing motion is a full-body technique involving multiple segments of the body going through multiple planes. The kinetic chain is vital for the effective execution of the throwing motion. Current Training Techniques

There is currently limited evidence pertaining to off-season sport-specific training for softball. ${ }^{10}$ There have been a number of studies that have examined the effect of types of exercises on throwing outcomes, including plyometric and closed kinetic chain glenohumeral joint complex exercises. ${ }^{12,}{ }^{19}$ However, these studies have examined only a single aspect of throwing or applied the intervention to a single body part, which is commonly the glenohumeral joint complex. Current practice advocates for a global approach to rehabilitation and strength of conditioning, as the lower extremity, trunk, and glenohumeral joint complex work together through a kinetic chain rather than as isolated segments. ${ }^{2,10}$ Even with this principle guiding rehabilitation, little evidence exists that examines the effect of a global training program has on performance variables. One study ${ }^{10}$ was published that suggested an off-season training program for softball athletes, but it did not study the effect this program had on performance variables of hitting or throwing in softball. One report for baseball in-season practices includes much of the 
same exercises and progressions. It involves isolated segment strengthening but also incorporated rotator cuff strengthening as oppose to the programs designed for softball. ${ }^{11}$ Furthermore, it involved the strengthening of the spinal segments and used explosive movements rather than isotonic progressions. The program utilized alternated intensity on days, with heavy strengthening days involving no sport specific movements and light days involving explosive movements through sport specific movements. ${ }^{11}$ This program was developed for collegiate baseball pitchers, but the techniques and progressions utilized in this contrast with those proposed for softball. ${ }^{10,11}$ This represents only one training regimen utilized for the overhead throwing motion in baseball, however.

Though evidence pertaining to specific training programs for softball is sparse, there exists a clear discrepancy between strength and conditioning practices in baseball. A survey of major strength and conditioning specialists who work in Major League Baseball revealed very different approaches to conditioning programs. ${ }^{48}$ In this study, 21 strength and conditioning coaches responded to the survey, and although many used some of the same practices, there were other areas that were conflicting. Twenty of the twenty one coaches reported using plyometric training, but only three coaches used Olympic style lifts in the programs. Nineteen reported that they will cycle the loads, meaning that the programs will go through phases that will cycle with different volumes and intensities. The amount of load is very subjective, and intensity and resistance for specific exercises is generally by a 'trial and error' process. Coaches also differed greatly on the most important exercises. Rotational core movement was seen as the first, second, third, fourth, and fifth most important exercise. Squats and lunges were seen as the first and third most important exercise. According to strength coaches, glenohumeral joint complex stabilization and rotator cuff strengthening was seen by many coaches as the least important 
exercise during training, reported by four coaches each. ${ }^{48}$ This conflicts directly with most rehabilitation schemes and prescribed treatments for glenohumeral joint complex rehabilitation.

Glenohumeral joint complex training techniques: Traditional rehabilitation and strengthening have focused on strengthening of isolated muscle groups, often in a single plane of motion. ${ }^{15}$ Strategies such of these are hardly functional, and do not address muscle coordination and sequencing that is indicative of athletic movement. Glenohumeral joint complex training techniques should be performed in a manner that improves strength, neuromuscular control, and functional performance. Strength in the glenohumeral joint complex is needed to improve dynamic stability of both the glenohumeral joint and scapula thoracic joint and provide normal function and movement while maximizing the potential for effective performance. Proper scapular upward rotation is necessary for biomechanically sound throwing technique. Strengthening scapular stabilizing muscles such as the lower trapezius is a common feature of rehabilitation protocols for the overhead thrower. In fact, it has been established that there is a good relationship between lower trapezius strength and the degree of scapular upward rotation. ${ }^{49}$ Neuromuscular control is especially important in the glenohumeral joint complex, as it has a delicate balance between mobility and stability. The neurological coordination and feedback mechanisms of the glenohumeral joint complex serve to promote effective movement and also serve to protect against excessive strain to the passive and dynamic restrains of the glenohumeral joint comple.

One such study by Padua and colleagues ${ }^{15}$ compared the effects an open kinetic chain (OKC), closed kinetic chain (CKC), and proprioceptive neuromuscular facilitation (PNF) exercise on functional throwing accuracy, neuromuscular control, joint position sense, and isokinetic strength testing. What was found was there was a significant increase in strength in the 
PNF and OKC groups, but not the CKC. Joint position sense and neuromuscular control was not affected by any of the exercise regimes. Functional throwing accuracy performance, however, was improved in the PNF group only. ${ }^{15}$

Another study also evaluated the effect closed kinetic chain exercises had on neuromuscular control, and had conflicting results to Padua et al. ${ }^{14}$ This was perhaps due to differences in methodology and testing procedure, but nonetheless, found that closed kinetic chain exercises had significant effects on neuromuscular control. Closed kinetic chain exercises have been described as superior to open kinetic chain exercises in that they force the subject to increase strength while balancing compressive and shear forces of the glenohumeral joint complex that occur during functional tasks and activating co-contraction of glenohumeral joint complex musculature. ${ }^{15}$ These actions together have been purported to increase neuromuscular control by forcing joint approximation that stimulates afferent neural feedback. ${ }^{15}$ There was another study ${ }^{12}$ conducted that the effect of closed kinetic chain exercises in Division I softball athletes. Outcome variables in this study were throwing velocity as well as isokinetic strength measures. What was found was that only closed kinetic chain exercises improved throwing velocity, but both groups had equal strength gains isokinetically. This study confirmed the effectiveness of closed kinetic chain exercises as a viable option of training for the overhead thrower.

Plyometric exercises have also been suggested for overhead throwing athletes, as ballistic movements are most sport-specific. Pezzullo and colleagues ${ }^{19}$ suggested a program of plyometric exercises into rehabilitation and strength programs to improve outcomes. These exercises mimic the arm cocking, arm acceleration, and arm deceleration phases of throwing. The exercises suggested are purely in the open chain, but can be altered to incorporate functional patterns of 
throwing. The intent of plyometric exercises is to increase power and adapt the glenohumeral joint complex to the stresses of overhead throwing. ${ }^{19}$ Commonly, these exercises are prescribed during the latter stages of rehabilitation and strength training. It has been shown that plyometric training yields no strength benefit to traditional training, but throwing velocity did increase. ${ }^{17}$

Comparative efficacy between more than two strengthening protocols is rare in the literature, especially when it comes to the effects on performance outcomes. However, DeRenne and colleagues ${ }^{13}$ reviewed how general, specific, and special training affected throwing velocity. General strengthening referred to those exercises that aim to increase the overall strength of the muscles. Commonly, these are isotonic exercises including the bench press or lat pulldown. It was reported that this type of training has conflicting evidence, where one study reported an increase in throwing velocity of only 0.62 miles per hour where two other studies reported increases in throwing velocities of 2.3 and 4.1 miles per hour. ${ }^{13}$ Special training refers to ballistic movements and muscle contractions that are similar to those in the overhand throwing motion. Only one study evaluated its efficacy, and had results that were unclear. There was an increase in throwing velocity, but it was unclear whether it was the result of the plyometric training or from the movement specific pattern. Special resistance training has been shown to be an effective method of increasing throwing velocity, but it was not shown what the effect on throwing velocity was. Increases in throwing velocity were reported as high as 3.75 miles per hour, and have been reported to be one of the best ways to increase throwing velocity. ${ }^{13}$ The mechanisms by which it improved throwing velocity, however, were not clearly defined.

Core training techniques: Specific exercises to improve core strength have shown even more variability than those addressing the effect core strength has on performance. Traditionally, core exercise tend to progress from static to unstable surfaces and finally to core training 
incorporating dynamic movement. Consistent training exercise and principles are lacking in the literature, with many different regimes (i.e. sling based training vs. Swiss ball) and exercises being used in order to train the core ${ }^{16,32,37,38,50,51}$ The lack of consistency is one of the main reasons why interpreting results is a difficult task when reviewing the plethora of articles that exist pertaining to core stability and performance. ${ }^{30,31,35,42}$

Though a lack of core training protocols exist in the literature, there are a few fundamental principles that have become commonplace. One of the most agreed-upon fundamental components of a core strengthening program is to first identify the deficiencies that an individual may have. ${ }^{37}$ By doing so, specific exercises can be determined in order to theoretically improve performance. ${ }^{37}$ Furthermore, motor control stability must be addressed, namely because the core is also responsible for the controlling of movement and not just the initiation of it. ${ }^{37}$ By allowing the subject to become aware of the movement patterns necessary for proper technique, recruitment of muscles in isolation or in tandem can be trained to perform more efficiently. ${ }^{35}$ Usually this starts with low-threshold exercises that are endurance-based in order to allow the central nervous system to recognize and integrate the movement patterns properly. It has also been reported that this type of training is essential when focusing on the local musculature in order to limit muscular imbalances that may lead to improper technique but also injury, though global musculature must also be trained in this manner as well. ${ }^{47}$ Core strengthening is also a supposed important part of training and must abide by the overload principle to consequently see hypertrophic effects that allow for more powerful torque production as well as allow more powerful isometric contraction in the stabilization of the lumbar spine. One report on surface electromyography of core musculature revealed that only $60 \%$ of maximum voluntary contraction was needed to see a hypertrophic effect on the 
musculature whereas only $25 \%$ of maximum voluntary contraction was needed to see endurance benefits. ${ }^{35}$ All of these areas should be emphasized in a core stabilization program, and trainers should be aware of the quality of the movement rather than the quantity. ${ }^{37}$

The incorporation of these principles into strength training programs is where the inconsistency occurs. One of the most common techniques used is the use of unstable surfaces in order to increase the demand placed on the core musculature to maintain integrity, although the efficacy of this method has been brought into question. ${ }^{37,40,52} \mathrm{McGill}^{\text {et al }}{ }^{37}$ argues that stability has little to do with the ability to balance on a gym ball, which only involves the ability to maintain the body in balance, which may not specifically address lumbar spine stability. In an analysis of muscle activation during Swiss ball exercises, it was found that there was a greater activation of muscles during Swiss ball exercises when compared to a stable surface. ${ }^{50}$ More precisely, different exercises showed different recruitment and synergistic patterns of the core musculature, and Swiss ball training can directly influence those relationships. Exercises such as the quadruped exercise was effective in targeting the local musculature while the press-up increased global musculature. ${ }^{50}$ Influence of unstable training causing a direct change in performance has been much less clear. Parkhouse et $\mathrm{a}^{51}$ provided little evidence to the training effect unstable surfaces give as opposed to stable surfaces. What was interesting, though, was that those subjects that performed static exercises improved significantly in static balance tests, but not in the dynamic activities, suggesting that there is a limited transfer of static core exericses to dynamic performance. ${ }^{51}$ The dynamic exercise group did not have significant improvement over the static exercise group, but improvement was noticed that may be clinically relevant. ${ }^{51}$ The lack of improvement found in the dynamic test group was rationalized by the fact that the exercises performed in training were not specific to the tasks they were asked to accomplish, 
which is an argument proposed by other research. ${ }^{35,37,51}$ It is suggested that core stabilization and strength training must abide by the specificity principle, as movement patterns during core training must mimic those patterns that occur during functional tasks. The lack of specificity might be the reason why few definitive results have been seen. ${ }^{42}$

Lower extremity training techniques: Lower extremity strengthening for the overhead throwing motion is not well researched, and only few studies have ascertained common practices and functional exercises for the baseball pitch. Currently, strength and conditioning coaches in Major League Baseball employ traditional isotonic exercises, including squats, lunges, and plyometrics, although the comparative importance of these exercises is variable among the practices. ${ }^{48}$ Plyometric training has received attention in the literature as well. Neuromuscular adaptations and improvements in functional outcomes have been implicated. It has been suggested that plyometric exercises increase neural drive to agonist muscles and improve intersegmental muscular coordination. ${ }^{53}$ Although these are most commonly prescribed lower body exercises, some functional exercises have been suggested, including medicine ball swing hops, lateral ground hops, and drop back lunges. The exercises developed relate to the biomechanics of pitching and mimic the lower extremity functions during a baseball pitch. ${ }^{23}$ Kinetic chain training techniques:

The coordination and direction of muscle contraction during functional motions is a learned skill, both in terms of the types of muscle contractions and the neural framework that guides it. As with any other exercise, enhancement of the kinetic chain should be done by incorporating it into exercise to impose muscle activation patterns found in the desired activity. It is thought that motor programs exist for movement patterns, rather than for motor programs existing for individual segments throughout the movement. As such, neglecting a segment or over- 
emphasizing another can cause breaks in the kinetic chain and have detrimental effects to the athlete and increase loads to the glenohumeral joint complex or distal segments. ${ }^{4}$ Focus on the neural framework during kinetic chain exercises allows for the restoration of normal motion through natural movement patterns. ${ }^{5}$ Kinetic chain rehabilitation focuses on the linkage system of the body's segments and the proximal-to-distal neural framework of the body to enhance appropriate glenohumeral joint complex motion. ${ }^{5}$ There has been sport-specific training used, especially with the use of weighted baseballs. ${ }^{13}$ Overall, however, it has been shown that specific resistance training may be the best way to increase throwing velocity. ${ }^{13}$ Increased neuromuscular control through the segments of the kinetic chain will allow for synergistic action in the muscles used in the throwing motion. Through this, proper energy transfer and force distribution can be achieved from the hips to the trunk to the glenohumeral joint complex to the hand and finally to the ball. The kinetic chain is the key to efficient and safe movement, creating an environment within the body's muscular system that permits dynamic motion.

Summary

Softball is an ever-growing sport, with a large amount of interest within the past few decades. It involves many dynamic activities incorporating multi-joint and multi-planar movements, including the overhead throwing motion. The throwing motion involves a sequential ordering of events or phases. Each phase consists of specific movement patterns that help achieve high ball velocity. Inconsistencies and deficiencies within these phases can cause injury and decreased performance. Inherent deficits in range of motion and strength can alter these phases. There are three segments instrumental to the throwing motion. The glenohumeral joint complex, the core, and the trunk all have specific functions needed to execute a proper throw. The kinetic chain is the synergistic relationship between these segments, and this relationship is 
the key to safely executing dynamic movement. Proximal to distal muscle activation patterns reflect the relationship between all segments of the throwing motion. Training techniques for the glenohumeral joint complex, core, and lower extremity are varied in the literature. Common practice rarely employs the kinetic chain into training and rehabilitation regimens. 


\section{APPENDIX C}

\section{ADDITIONAL METHODS}

Table C1. Informed consent

\section{Only Minimal Risk} Consent Information and HIPAA Form

Principal Investigator

Department

Study Title

Performance Outcomes in Softball

Co-Investigator(s)
Dr. Michelle A Sandrey

College of Physical Activity and Sport Sciences

The effects of a Funtional Training Program on

Zachary M Mohondro ATC

\section{Contact Persons}

In the event you experience any side effects or injury related to this research, you should contact Dr. Michelle A. Sandrey at (304) 293-0870. If you have any questions, concerns, or complaints about this research, you can contact Zachary M Mohondro ATC at (410) 259-5704 or Dr. Michelle A Sandrey at (304) 293-0870.

For information regarding your rights as a research subject, to discuss problems, concerns, or suggestions related to the research, to obtain information or offer input about the research, contact the Office of Research Compliance at (304) 293-7073.

In addition if you would like to discuss problems, concerns, have suggestions related to research, or would like to offer input about the research, contact the Office of Research Integrity and Compliance at 304-293-7073.

\section{Introduction}

You, have been asked to participate in this research study, which has been explained to you by Zachary M Mohondro ATC. This study is being conducted by Dr. Michelle A. Sandrey in the College of Physical Activity and Sport Sciences at West Virginia University. This research is being conducted to fulfill the requirements for a Thesis in Athletic Training in the College of Physical Activity and Sport Sciences at West Virginia University under the supervision of Michelle A Sandrey, PhD, ATC.

2014.01.06.01
Protocol\#:
Approved: $\frac{2.11 \cdot 14}{2 \cdot 10 \cdot 15}$
Expires: $\frac{2 \cdot 10}{3 \pi C}$
Authorized:




\section{Purpose(s) of the Study}

The purpose of this study is to evaluate the effectiveness of a sport-specific training program on throwing speed and throwing accuracy. It also will attempt to evaluate the effect on arm control and awareness of the arm in space.

\section{Description of Procedures}

Before the commencement of the study, an orientation meeting will provide you the purpose and methods of this study. At this meeting, risk, discomforts, and confidentiality issues will be explained to you and your consent and demographic information will be obtained. Following this orientation, pre-test measures will commence.

You will be tested using the Closed Kinetic Chain Upper Extremity Stability Test, Functional Throwing Performance Index (FTPI), throwing velocity test, and joint positioning testing. Testing will be administered and supervised by the principal investigator at Waynesburg College $51 \mathrm{~W}$. College Street, Waynesburg, PA 15370. Prior to testing, all devices and procedures will be explained to your satisfaction. The total time for this study will be approximately 8 weeks. There will be one week in the beginning for pre-testing procedures, followed by 6 -week training program, and another week for post-test procedures.

Functional Throwing Performance Index

You will warm up on a treadmill for 5 minutes at a self-selected pace followed by stretching of your rotator cuff, biceps, and triceps for 5 minutes. You will then take a five minute break. Next you will be instructed to go through my normal throwing warm up routine. You will be instructed to stand 15 feet from a $1 \mathrm{ft} \times 1 \mathrm{ft}$ box, 4 feet high off the floor. Using your natural overhead throwing motion, you will throw the ball under control and as fast and accurately as possible, while catching the thrown ball's rebound as quickly as possible. You will be given the chance to practice the test with a $25 \%, 50 \%$, and $75 \%$ submaximal warm up throws, each trial lasting 30 seconds. You will start the test on the "go" command. You will perform three 30 second tests in which you will throw as many times as possible with control and accuracy. You will receive a one minute rest period in between each trial. The total number of throws and the total number of accurate throws will be counted from each of your three trials. Those throws landing within the target square, not on the line, will be defined as accurate throws. The principal investigator will determine percentage scores for each trial by dividing the number of accurate throws by the total number of throws you perform. The average percentage scores from your three trials will then be calculated and recorded. Following the test results will be recorded on a data sheet. If all data had been correctly recorded, your testing session will be completed. At this time you will be asked by the primary researcher for any questions or comments. You will then be given a 5 minute rest period before beginning the next test.

Throwing Velocity Test

After the functional throwing performance index, you will then participate in the throwing velocity test. You will take 3 submaximal warm-up throws at $75 \%$ effort.

2014.01 .06 .01

Protocol\#:

Approved: $2 \cdot 11 \cdot 14$

Expires: $\frac{2 \cdot 11 \cdot 15}{2 \cdot 10 \cdot 15}$

Authorized: BRC 
You will then rest for 5 minutes. After, you will take three throws at full

volitional capacity and try to achieve the fastest throw that you can.

The throw will targeted at a $3 \mathrm{ft} \times 4 \mathrm{ft}$ box on the wall.

Your throws will be measured by a standard radar gun. After, you will get a 5 minute

rest period before beginning the next test

Upper Extremity Stability Test

You will assume a push-up position with each of my hands being placed on a piece of tape 1.5 inches in width and 36 inches apart from each other. You will begin the test when the "go" command is given removing either hand from the strip of tape and touching the other piece of tape with that hand. You will be given a submaximal test to familiarize myself with the test. You will then perform 3 maximal tests for 15 seconds each. Next, you will then be given a 45 second break in between each test. Following each test, the primary investigator will record the data and average the 3 maximal test scores together to create your test score. Once all the data has been recorded correctly, this test will be finished.

Joint Positioning Testing

To begin, you will lie on your back on a table with your arms at your sides.

The primary investigator will bring your arm into a pre-determined position and ask you to remember where this arm is in space. The primary investigator will then place your arm back at my side in its resting position. You will then move your arm back, without direction, to the position that the primary investigator placed it in. The primary investigator will then measure the absolute error (if any) of your arm for both abduction and internal/external rotation. At this time, you will be asked by the primary researcher for any questions or comments.

Following pretest procedures, you will undergo a six week functional training program. The exercises will be those that have been recommended through the literature and practice that will improve strength, control, balance, and endurance. They are exercises that will include thera-bands and dumbbells and will be directed towards the hip, core, and shoulder. The exercises will increase in difficulty from week to week. All exercises will be explained and shown by the principal investigator. A packet of exercises will be provided for your personal reference.

Those in the control group will only perform the pre and post testing procedures.

\section{Discomforts}

There are no known or expected risks from participating in this study. Discomforts from this study may include some soreness in your upper extremity, lower extremity and core following the strengthening exercises, but should be minimal. I will perform stretches of the arms and legs to decrease soreness. Should any injury occur, you

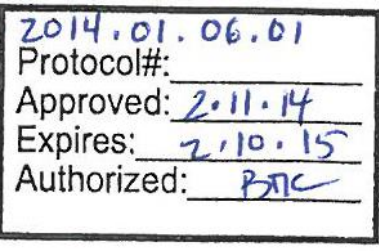


understand that Zachary M Mohondro, ATC will provide first aid and make any necessary medical referral at my expense.

\section{Alternatives}

You do not have to participate in this study and will continue with usual softball

activities under the direction of your coach.

No negative action will be taken against you if you choose not to participate in this study.

\section{Benefits}

Benefits from this study may include increased strength and control of your arm. You may also see benefits in your overhead throwing ability in terms of throwing accuracy and throwing velocity.

\section{Financial Considerations}

You will not earn any financial compensation for participating in this study.

\section{Confidentiality}

Any information about you that is obtained as a result of your participation in this research will be kept as confidential as legally possible. Your research records and test results, just like hospital records, may be subpoenaed by court order or may be inspected by the study sponsor or federal regulatory authorities without your additional consent.

\section{HIPAA}

We know that information about you and your health is private. We are dedicated to protecting the privacy of that information. Because of this promise, we must get your written authorization (permission) before we may use or disclose your protected health information or share it with others for research purposes.

You can decide to sign or not to sign this authorization section. However, if you choose not to sign this authorization, you will not be able to take part in the research study. Whatever choice you make about this research study will not have an effect on your access to medical care.

\section{Persons/Organizations Providing the Information}

USE AND DISCLOSURE COVERED BY THIS AUTHORIZATION. DO NOT SIGN A

BLANK FORM. You or your authorized representative should thoroughly

2014.01.06.01
Protocol\#:
Approved: $\frac{2.11 \cdot 14}{2 \cdot 10 \cdot 15}$
Expires: $\frac{2 \cdot 17 C}{\text { Authorized: }}$


read the information below before signing this form. This form will authorize the following person(s), class(es) of persons, and/or organization(s) to disclose, use, and receive the information: WVU, Dr. Michelle A Sandrey, PhD, ATC, and Zachary M Mohondro, ATC. The research site(s) carrying out this study includes WVU. If, during the course of the research, the institution listed above merges with, or is purchased by, another company or institution, this authorization to use or disclose protected health information in the research will extend to the successor, company or institution.

\section{Persons/Organizations Receiving the Information}

- $\quad$ The research site of this study is the Marisa Rudy Field House at Waynesburg

University, Waynesburg PA 15730

- $\quad$ Zachary M Mohondro ATC and Dr. Michelle Sandrey PhD ATC

- $\quad$ The United State Department of Health and Human Services

(which includes the National Institutes of Health $(\mathrm{NIH})$, Food and Drug

Administration (FDA)) and other groups that have the right to use the information as required by law.

- $\quad$ The members and staff of any Institutional Review Board

(IRB) that oversees this research study.

- West Virginia University Office of Research Compliance and Office of Sponsored Programs.

\section{The Following Information Will Be Used}

A self-reported demographic questionnaire will be used from this study. Pre and post test scores for throwing accuracy, throwing velocity, joint position sense, and upper extremity stability tests will be used. After all data has been analyzed, conclusions regarding the data and the effects will be disclosed with the participants.

\section{The Information is Being Disclosed for the Following Reasons}

- $\quad$ Review of your data for quality assurance purposes

- $\quad$ Publication of study results

- $\quad$ Other research purposes such as reviewing the safety or effectiveness

of the training program or; conducting performance reviews of the training program;

evaluating other training programs; developing a better understanding of training programs; improving the design of future clinical trials.

\section{You May Cancel this Authorization at Any Time by Writing to the Principal Investigator}

Dr. Michelle A. Sandrey, PhD, ATC.

College of Physical Activity and Sport Sciences

PO Box 6116

msandrey@mix.wvu.edu

2014.01 .06 .01

Protocol\#:

Approved: $2.11 \cdot 14$

Expires: $2,10 \cdot 15$

Authorized: BRC

(304) 293-0870 
If you cancel this authorization, any information that was collected already for this study cannot be withdrawn. Once information is disclosed, according to this authorization, the recipient may redisclose it and then the information may no longer be protected by federal regulations.

This authorization will expire at the end of the study unless you cancel it before that time (or has a specific expiration date).

\section{Voluntary Participation}

Participation in this study is voluntary. You are free to withdraw your consent to participate in this study at any time.

Refusal to participate or withdrawal will not affect your future care as appropriate a will involve no penalty to you or your position on the team.

In the event new information becomes available that may affect your willingness to participate in this study, this information will be given to you so that you can make an informed decision about whether or not to continue your participation.

You have been given the opportunity to ask questions about the research, and you have received answers concerning areas you did not understand.

Upon signing this form, you will receive a copy.

I willingly consent to participate in this research.

\section{Signatures}

Signature of Subject

Printed Name

Date

Time

The participant has had the opportunity to have questions addressed. The participant willingly agrees to be in the study.

Signature of Investigator or Co-Investigator

Printed Name

Date

Time

2014.01 .06 .01 Protocol\#:

Approved: $2 \cdot 11 \cdot 14$

Expires: $2,10 \cdot 15$

Authorized: $B \pi C$ 
Table C2. Subject Demographics

Subject Number:

Age:

Height:

Weight:

Gender: Male / Female

Dominant Arm: Right/Left

Injury History

1. Have you had an upper extremity injury within the past six months? Yes/No

If yes, Please explain:

2. Have you ever had a lower extremity injury within the past six months? Yes/No

If yes, Please explain:

3. Have you had a head injury within the past six months? Yes/No

If yes, please explain:

4. Have you had any neurological disorders within the past six months? Yes/No

If yes, please explain:

5. Have you had any vestibular disorders within the past six months? Yes/No

If yes, please explain:

6. Have you had visual disorders within the past months? Yes/No

If yes, please explain:

7. Are you currently involved in any of the following physical activities?

Weight Training/Cardiovascular Training/Pilates/Yoga/Other

If yes, please explain what physical activity you are involved in and how often you are

involved in each

activity:

8. Are you taken any medications that affect performance or balance? Yes/No

If yes, please explain:

9. Do you have a history of chronic shoulder instability? Yes/No

If yes, please explain:

10. Are you currently in a training program for balance? Yes/No

If yes, please explain:

11. Are you currently in a core stability program for balance? Yes/No

If yes, please explain:

12. Are you currently in a training program to improve performance? Yes/No

If yes, please explain: 
13. Have you had any surgeries on either hips, shoulders, or your abdomen in the past year? Yes/No If yes, please explain:

Table C3. Pre/posttest measures data collection sheet

Pretest / Posttest

Subject Number:

Date:

$\underline{\text { Joint Position Sense }}$

Trial 1 Abduction Error:

Trial 1 Rotational Error:

Trial 2 Abduction Error:

Trial 2 Rotational Error:

Trial 3 Abduction Error:

Trial 3 Rotational Error:

Average Abduction Error:

Average Rotational Error:

Average Total Error:

Functional Throwing Performance Index

Trial 1 total throws:

Trial 2 total throws:

Trial 3 total throws:

Average number of throws:

Index Score:

Throwing Velocity

Trial 1 Velocity:

Trial 2 Velocity:

Trial 3 Velocity:

Average Velocity:

\section{Closed Kinetic Chain Upper Extremity Stability Test}

Trial 1 number of touches:

Trial 2 number of touches:

Trial 3 number of touches:

Average number of touches:
Trial 1 accurate throws:

Trial 2 accurate throws:

$\%:$

$\%$ :

Trial 3 accurate throws:

$\%$ :

Average number of accurate throws: 
Week Exercise

$1 \quad Y^{\prime}$

T's

90/90 Thera-band external rotation

90/90 Thera-band internal rotation

Quadruped (single limb)

Single leg bridges

Hip 4-Way

Front Lunges

Side Lunges

2

Shoulder $\mathrm{D}_{1}$ PNF patterns with manual

resistance

Shoulder $\mathrm{D}_{2}$ PNF patterns with manual

resistance

Rhythmic Stabilization

Quadruped (double limb alternating)

Single leg Bridges on Airex

Front BOSU lunges

Side BOSU lunges

BOSU Squats

3

BOSU push-ups

Physioball push up balance

Leg Lifts with rotation

Double leg bridges on physioball

Medicine ball lateral swing hop

Lateral ground hop

$4 \quad$ Kneeling 90/90 ball toss forward

Kneeling reverse throw

Kneeling chop
Sets Reps Weight/Reisistance Target segment

$\mathrm{N} / \mathrm{A}$

$4 \mathrm{lb}$. medicine ball

N/A

$1 \mathrm{lb}$. ball

$1 \mathrm{lb}$. ball

Black thera-band
GHJ Complex

GHJ Complex

GHJ Complex

GHJ Complex

Core

Core

Lower extremity

Lower Extremity

Lower Extremity

GHJ Complex

GHJ Complex

GHJ Complex

Core

Core

Lower Extremity

Lower Extremity

Lower Extremity

GHJ Complex

GHJ Complex

Core

Core

Reference

20

20

19

19

16

16

2

16

-

Lower Extremity

Lower Extremity

GHJ Complex

GHJ Complex

GHJ Complex,
21 
Medicine ball rebound lateral toss Front drop back lunge

Plyometric lunge ground hop

5

Lunge punches

Lawnmower pulls

Stance Balance

$6 \quad$ Lift/Place with dumbbell

Standing lunge chop with rotation

Thera-band throwing motion with rotation

\section{Core}

$2 \quad 20 \quad 6 \mathrm{lb}$. medicine bal

Core

Lower Extremity

Lower Extremity

GHJ Complex,

Core, Lower

Extremity

GHJ Complex,

Core, Lower

Extremity

Lower Extremity

GHJ Complex,

Core, Lower

Extremity

GHJ Complex,

Core, Lower

Extremity

GHJ Complex,

Core, Lower

Extremity

16

22

22 
Figure C1. Pre/Post Test Measures

\section{Joint Position Sense}

1. Prior to testing the devices and procedures were explained to the subject.

2. The subject laid supine on the table.

3. The primary investigator moved the throwing arm to a position of ninety degrees glenohumeral abduction and ninety degrees glenohumeral internal rotation using a goniometer and asked the subject to remember where the arm is in space.

4. The primary investigator moved the limb back to a resting position at the side.

5. The subject attempted to move the arm back to the testing position.

6. The primary investigator measured the subject's arm position using a goniometer.

7. The primary investigator recorded the absolute value of the error for glenohumeral rotation.

8. These procedures were repeated three times for both arms and the average total error was used.
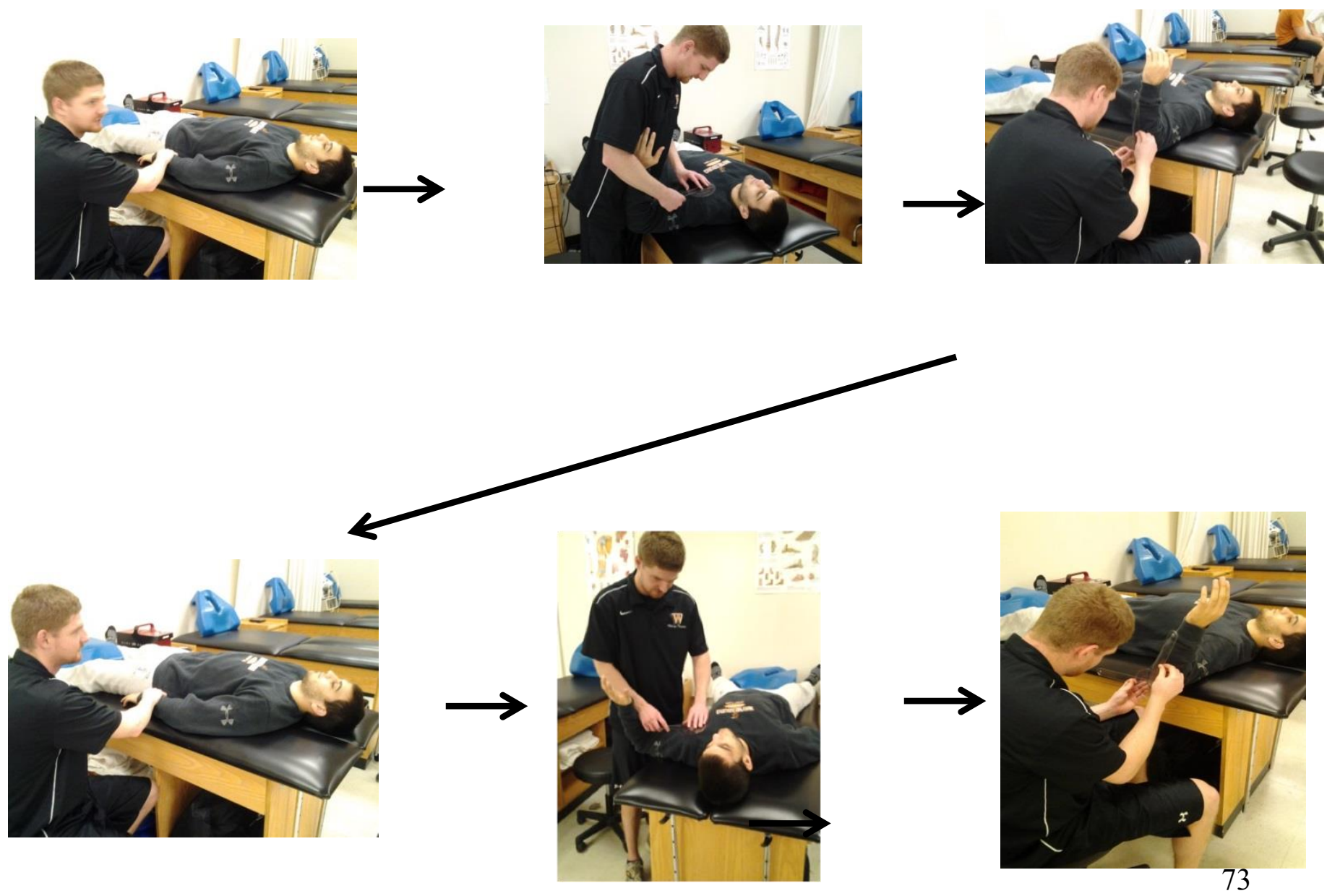


\section{Functional Throwing Performance Index}

1. Prior to testing the devices and procedures were explained to the subject.

2. The subject stood 15 feet away from a $1 \mathrm{ft} \times 1 \mathrm{ft}$ square taped on a wall that is $4 \mathrm{ft}$ off the ground.

3. The subject attempted to throw the softball into the square, grab the rebound, and repeat throwing the ball into the square.

4. The subject was given the chance to practice the test with a $25 \%, 50 \%$, and $75 \%$ submaximal warm up throws, each lasting 30 seconds.

5. After the warm up period, the subject performed the test and tried to throw as many times into the square in 30 seconds as possible.

6. This test was repeated three times and the average score was used for index calculation.
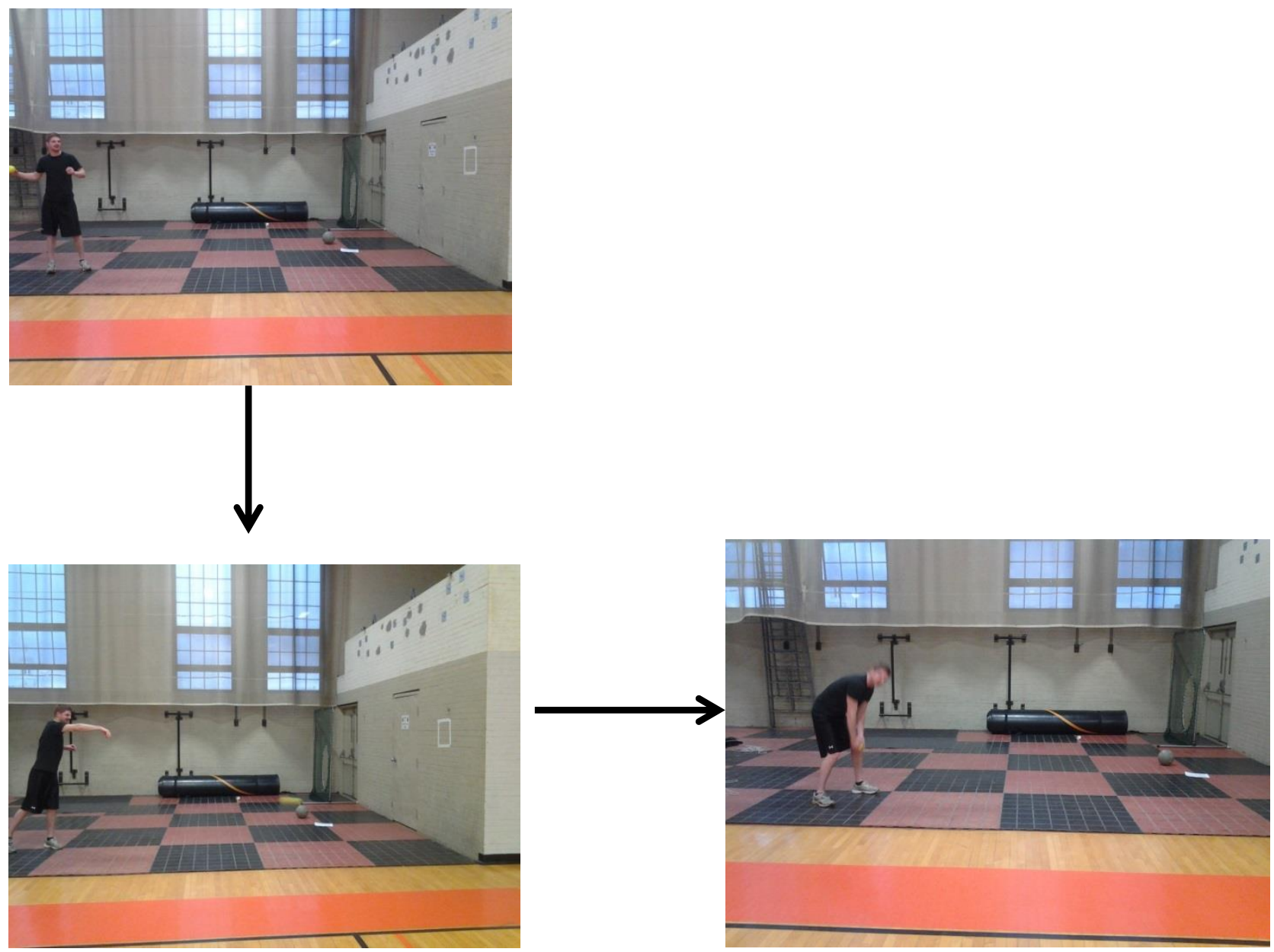


\section{Throwing Velocity Test}

1. Prior to testing the devices and procedures were explained to the subject.

2. The subject stood twenty feet away from a wall.

3. The subject warmed up and threw a softball at $25 \%, 50 \%$, and $75 \%$ the volitional capacity.

4. The subject performed three throws as fast as they could.

5. The primary investigator stood behind the subject with a radar gun and measured the velocity of the throw.

6. This was repeated three times and the average velocity was used.
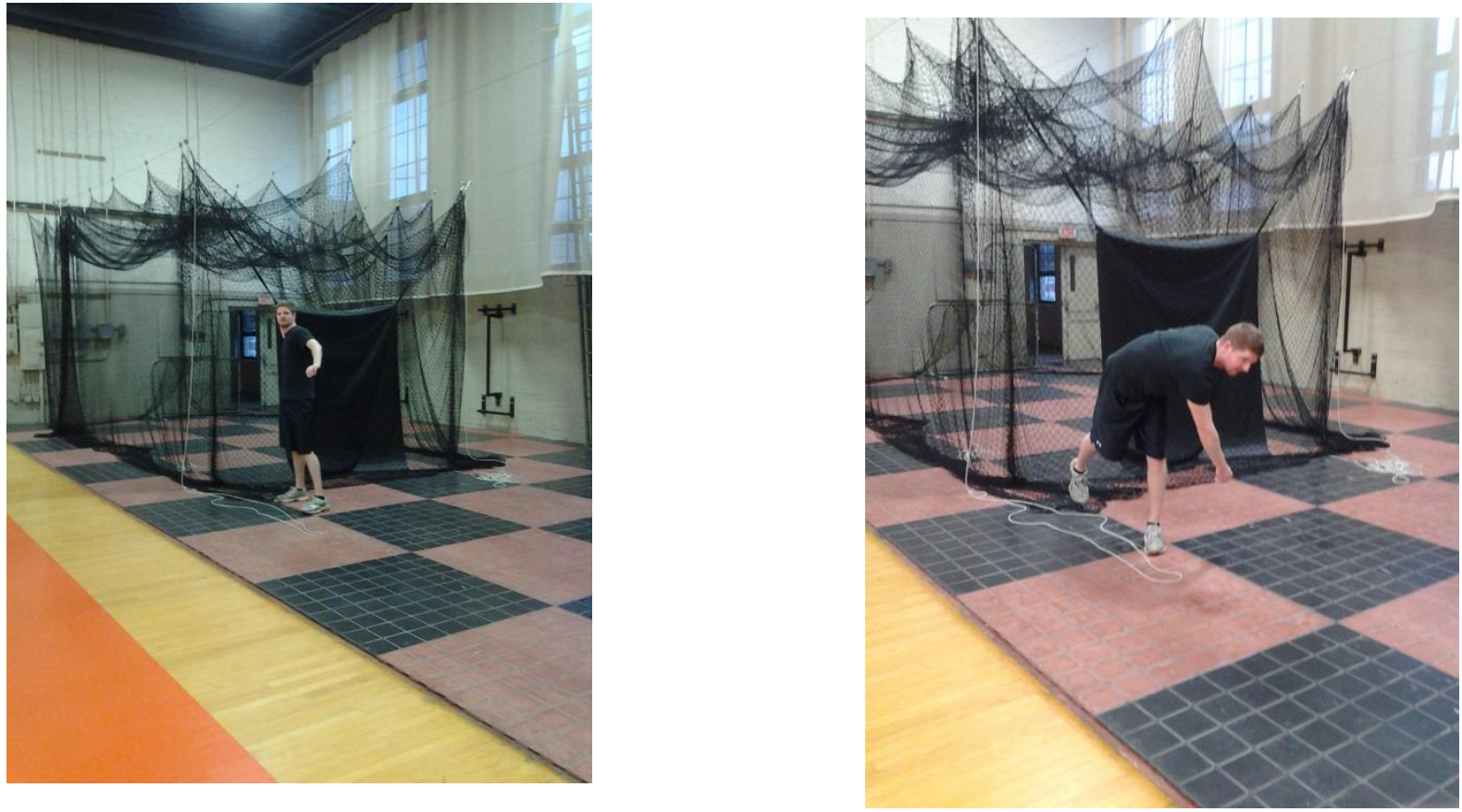


\section{Upper Extremity Stability Test}

1. Prior to testing the devices and procedures were explained to the subject.

2. The subject assumed a push-up position with the hands 36 inches apart and the hands placed on a piece of tape 1.5 inches in length.

3. The subject removed the non-dominant hand and placed it next to the dominant.

4. The subject re-placed the hand back to the starting position and then removed the dominant hand and placed it next to the non-dominant hand.

5. The subject tried to repeat this motion as many times as they can in 15 seconds for three trials.

6. The act of one arm crossing the midline and placing it down next to the other hand while maintaining form was defined as a 'touch'.

7. The average number of touches between the trials was used.

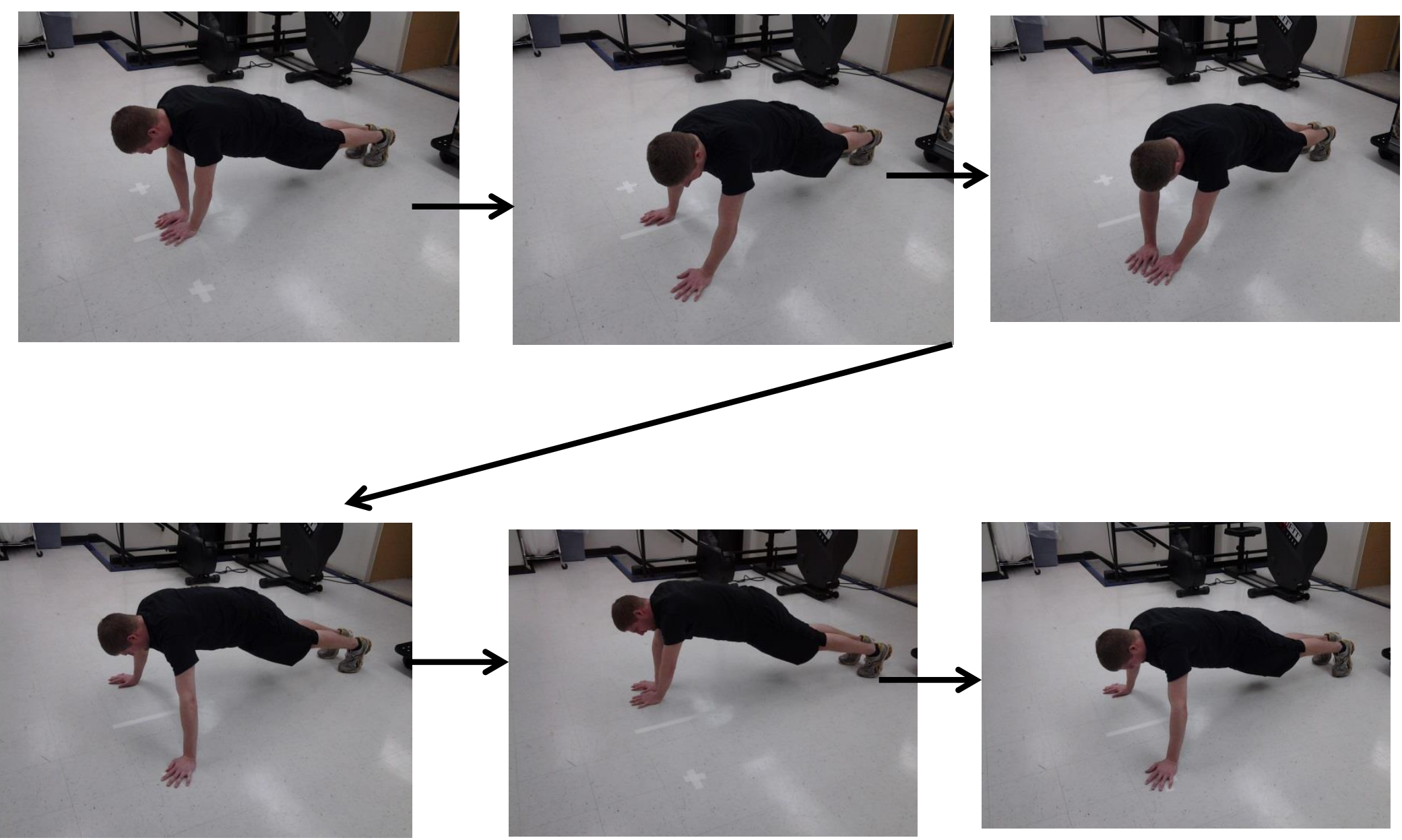


1. Subject laid prone on a table with the arm at 135 degree abduction and the arms hanging over the edge holding weight

2. The subject retracted the scapulas and raised the arms so they are in line with the body.

3. The subject lowered the arms and return to the starting position
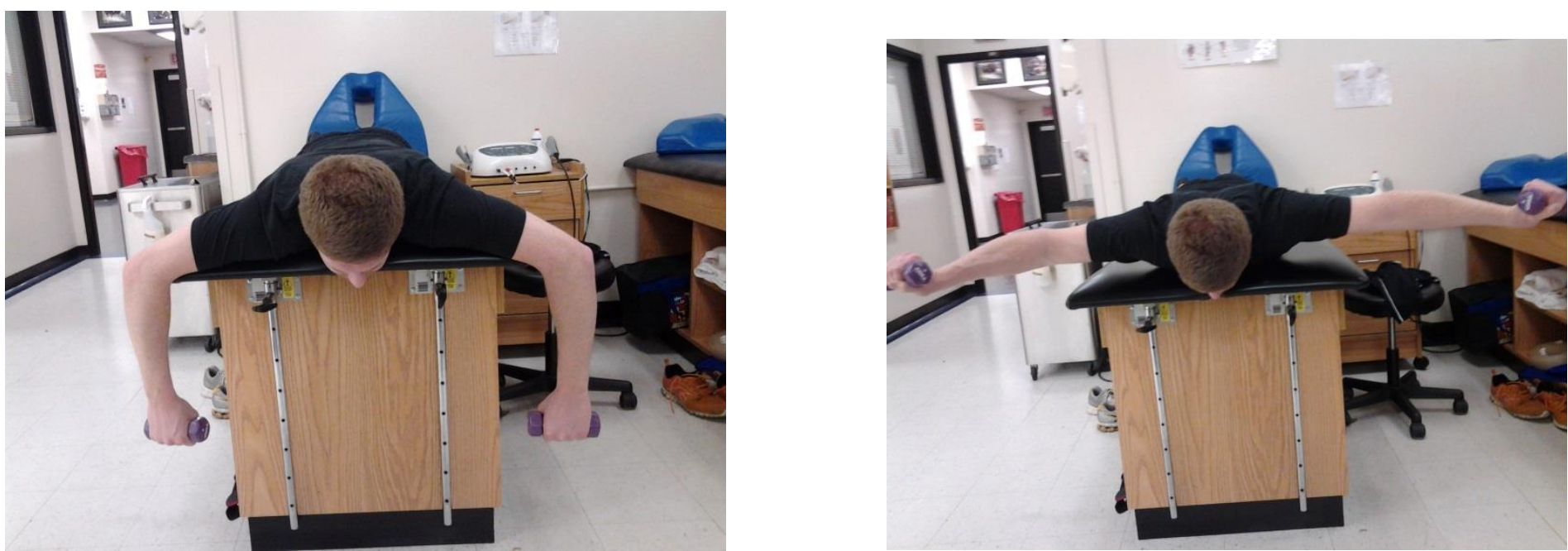

T's

1. The subject laid prone on a table with the arms abducted to 90 degrees with the arms handing off the edge of the table

2. The subject retracted the scapulas and raised the arms so they are in line with the body.

3. The subject lowered the arms and return to the starting position
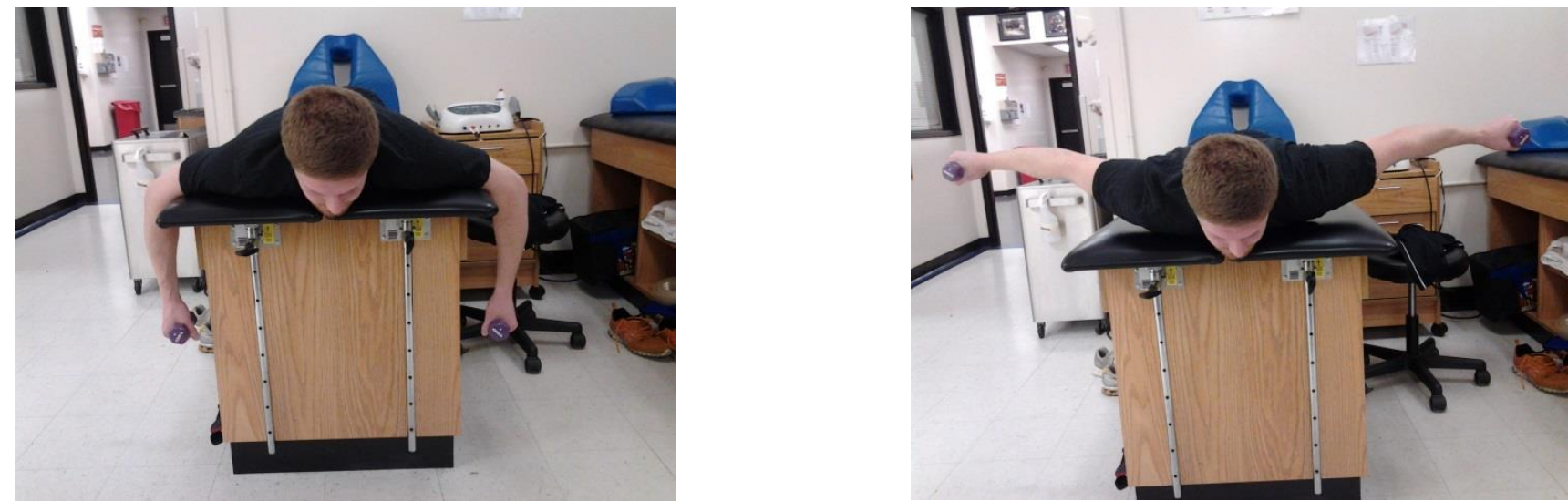
Thera-Band 90/90 External Rotation

1. The subject stood facing a fixed wall with the throwing shoulder abducted to 90 degrees, neutral rotation, and the elbow flexed to 90 degrees.

2. The subject gripped the end of a thera-band that is fixed to the wall directly in front of them at shoulder height

3. The subject externally rotated the arm until it reached a neutral position.

4. Subject lowered the arm back to the starting position

5. The subject did this for the throwing shoulder
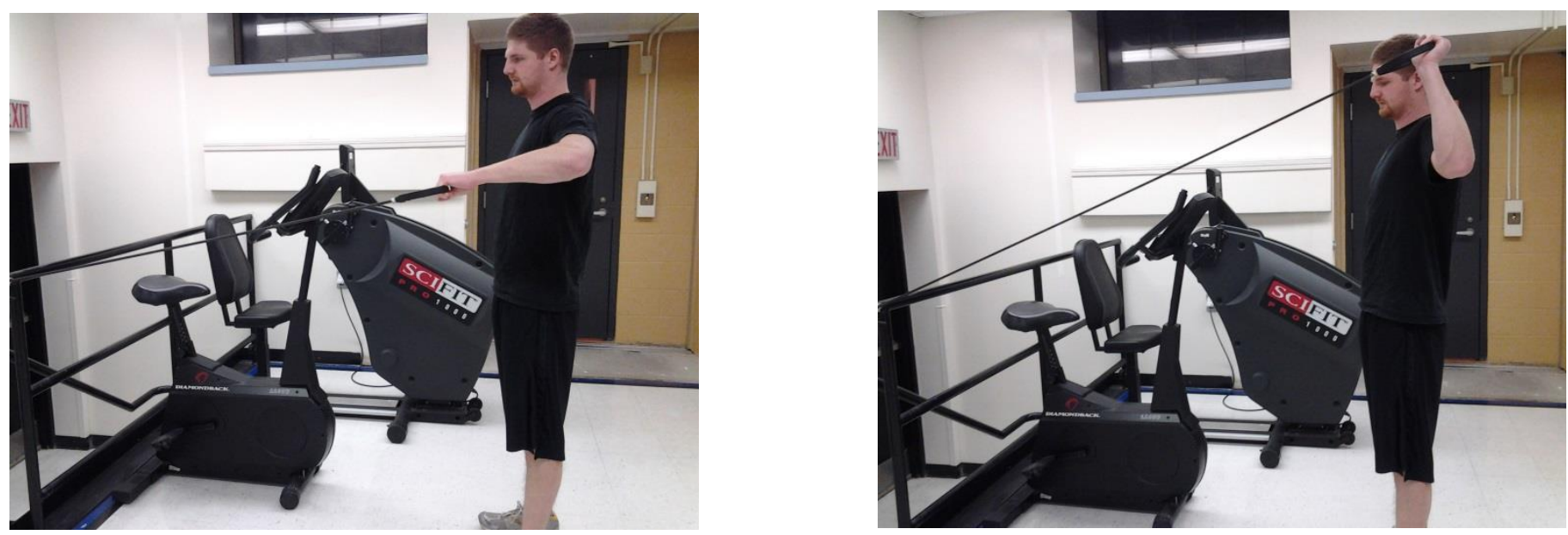

Thera-band 90/90 Internal Rotation

1. The subject stood facing a fixed wall with the throwing shoulder abducted to 90 degrees, externally rotated to 90 degrees and the elbow flexed to 90 degrees.

2. The subject gripped the end of a thera-band that is fixed to the wall directly in front of them at shoulder height

3. The subject internaly rotated the arm until it reaches 90 degrees of internal rotation.

4. Subject lowered the arm back to a neutral position

5. The subject did this exercise only with the throwing shoulder
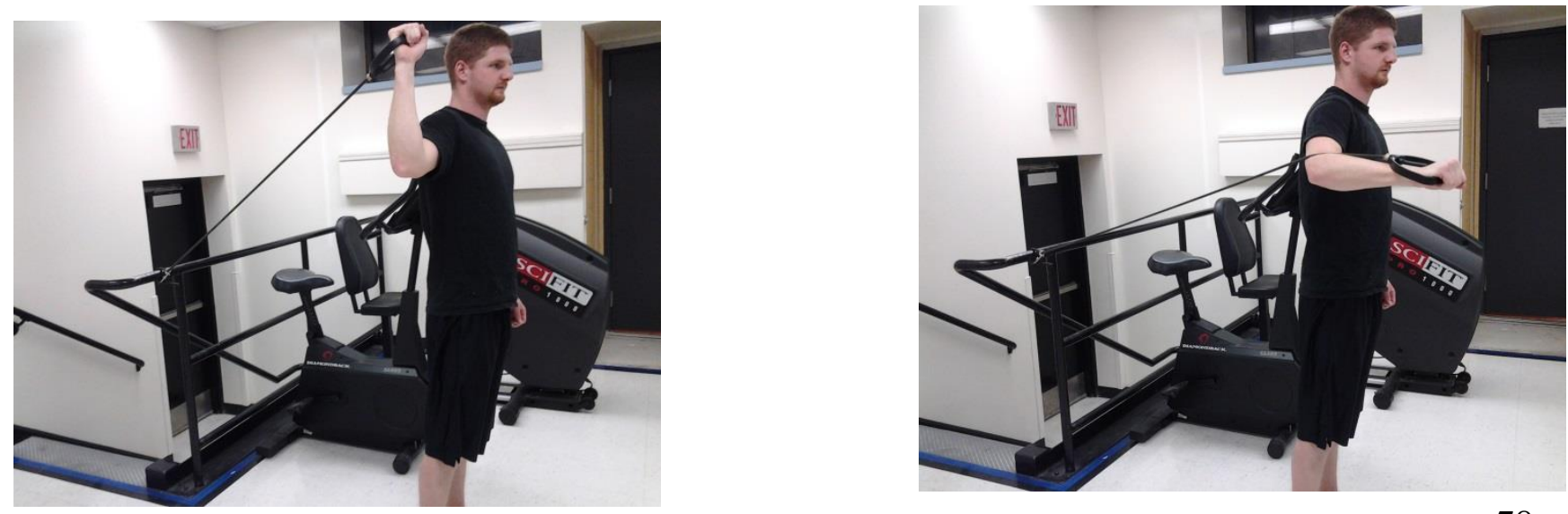


\section{Quadruped (Single Limb)}

1. Subject began on a treatment table on all fours.

2. While maintaining the balance, the subject raised the limbs, one at a time, until they were in line with the torso.

3. The subject lowered the limb to the original starting position.
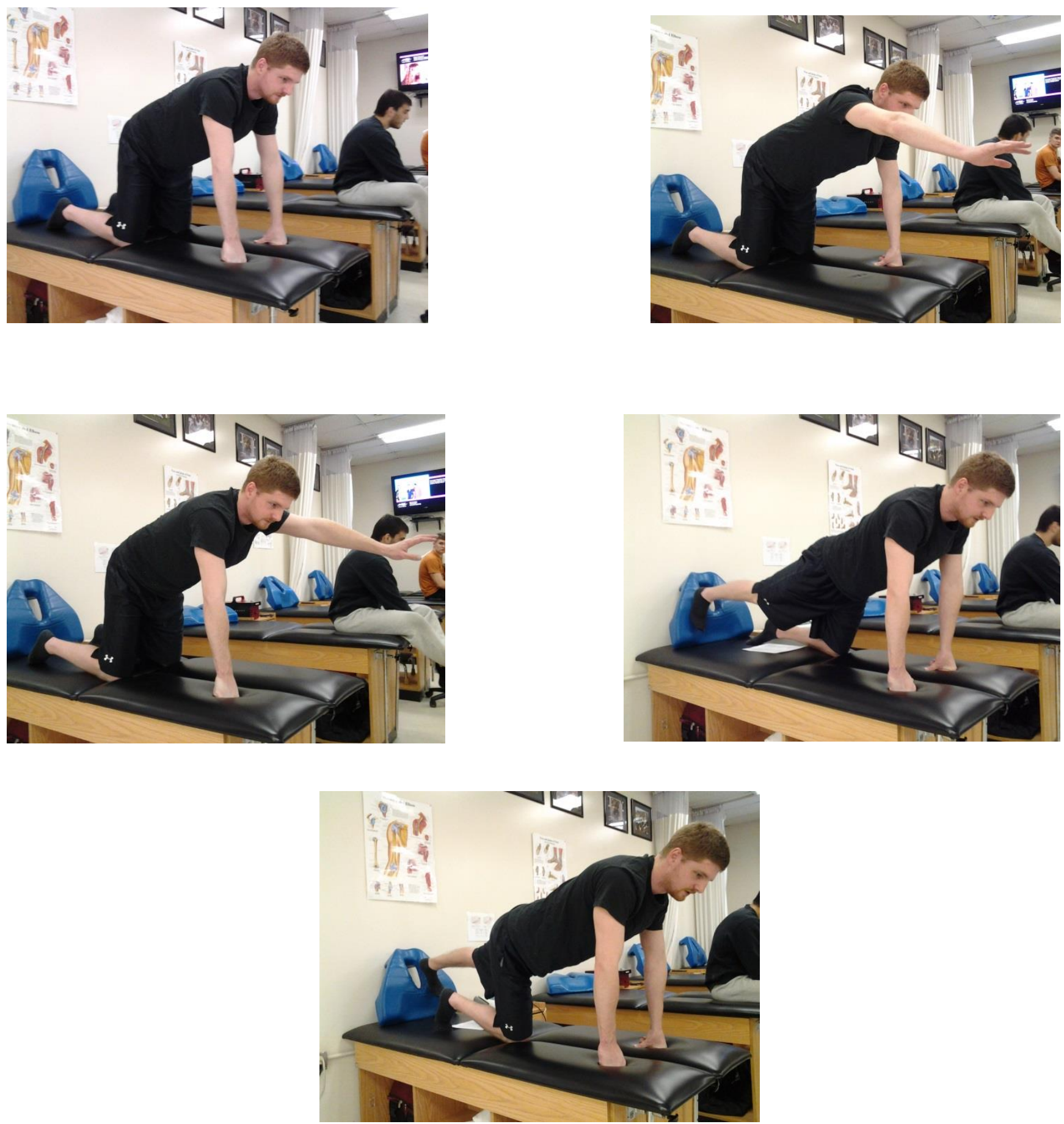
Single Leg Bridges

1. The subject laid supine on a table with the knees bent and the feet flat on the table.

2. The subject extended one hip so the shoulder blades remain on the table and the torso and extended hip leg are in line. The contralateral leg was extended at the knee.

3. The subject lowered themselves back into the starting position

4. This was done with both legs

Hip 4-Way
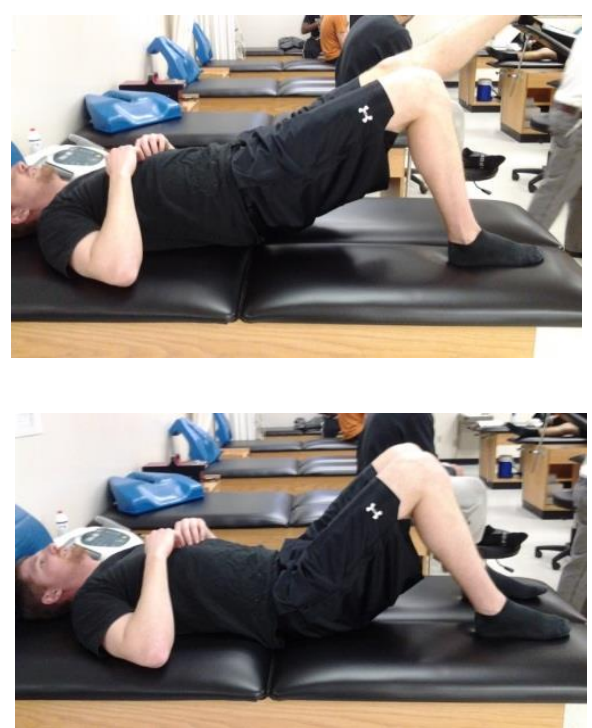

1. The subject stood facing a wall that has a length of resistance band attached to it at ankle height

2. The subject fixed the other end of the resistance band to the ankle

3. The subject stabilized the body with the contralateral leg and moved the entire ipsilateral leg into extension

4. The subject stood perpendicular to the wall so the ipsilateral hip was furthest from the wall, stabilized the body with the contralateral leg, and moved the hip into abduction

5. The subject stood facing away from the wall, stabilized the body with the contralateral leg, and moved the hip into flexion

6. The subject stood perpendicular to the wall so the ipsilateral hip was closest to the wall, stabilized the body with the contralateral leg, and moved the hip into adduction

a. All motions completed with both legs
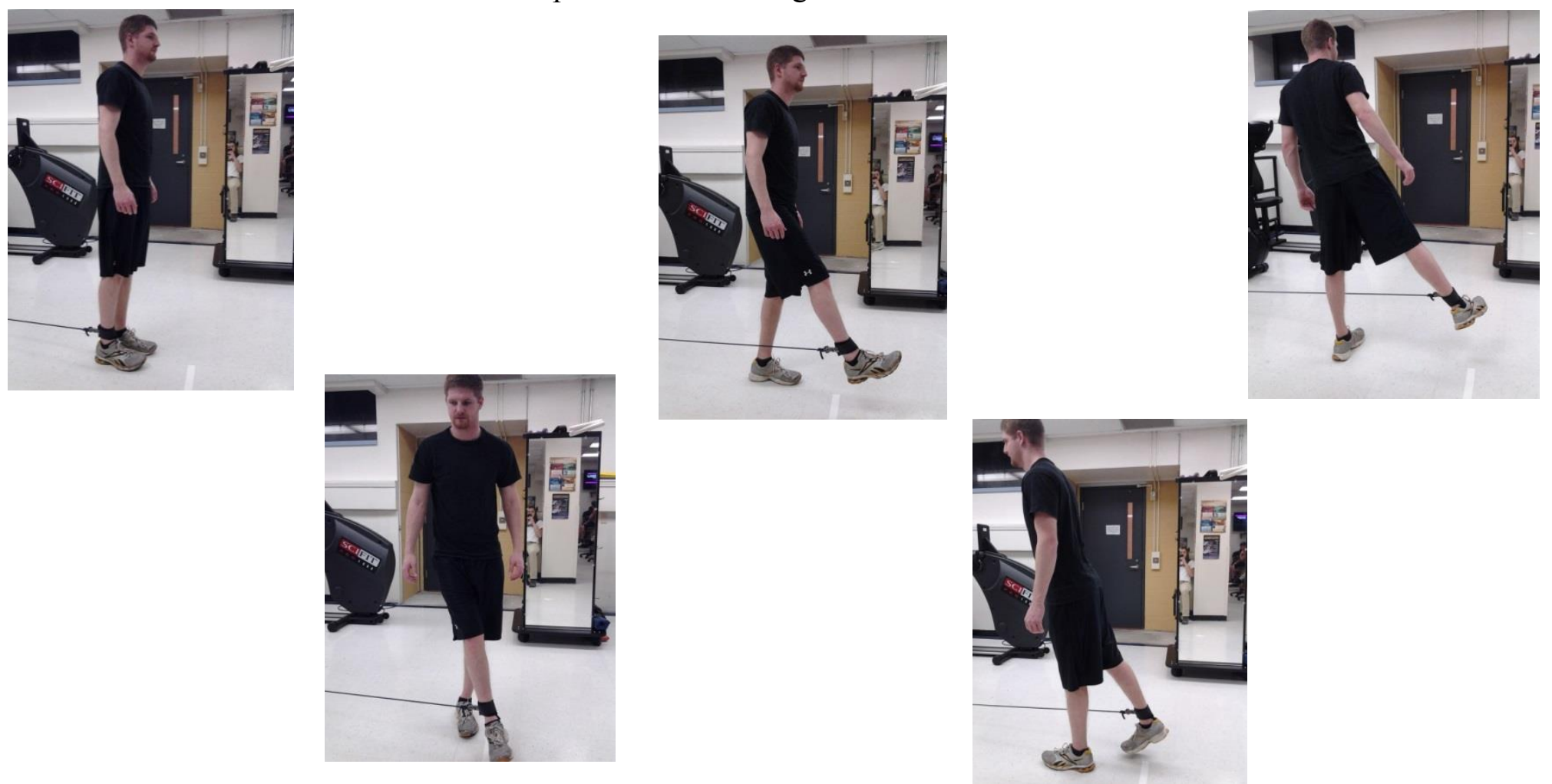


\section{Front Lunges}

1. Subject stood upright in the middle of an empty space

2. While maintaining an upright position of the torso, subject stepped with the stride leg into flexion until the stance leg was parallel to the floor

3. Subject stepped back to the starting position

4. This was only performed for the stride leg
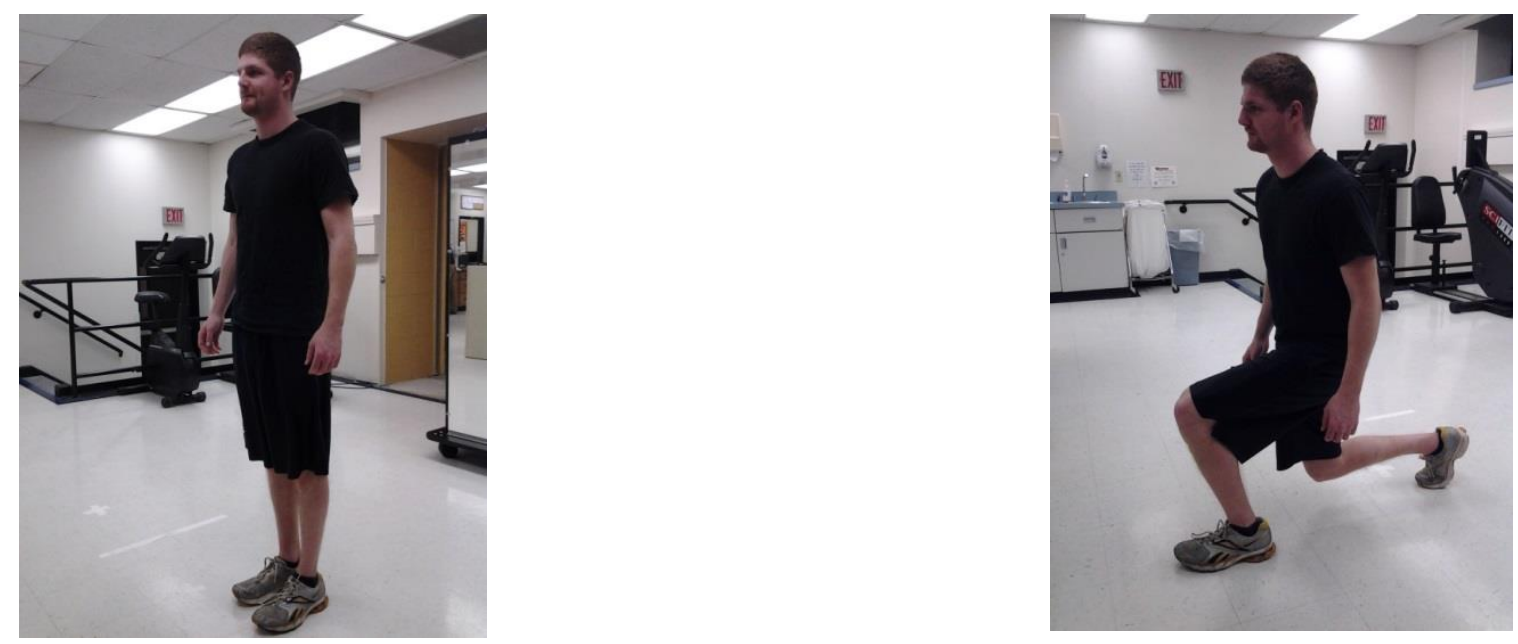

Side Lunges

1. Subject stood upright in the middle of an empty space

2. While maintaining an upright position of the torso, subject stepped into abduction with the stride leg until the femur was parallel to the floor

3. Subject stepped back to the starting position

4. This was only performed for the stride leg
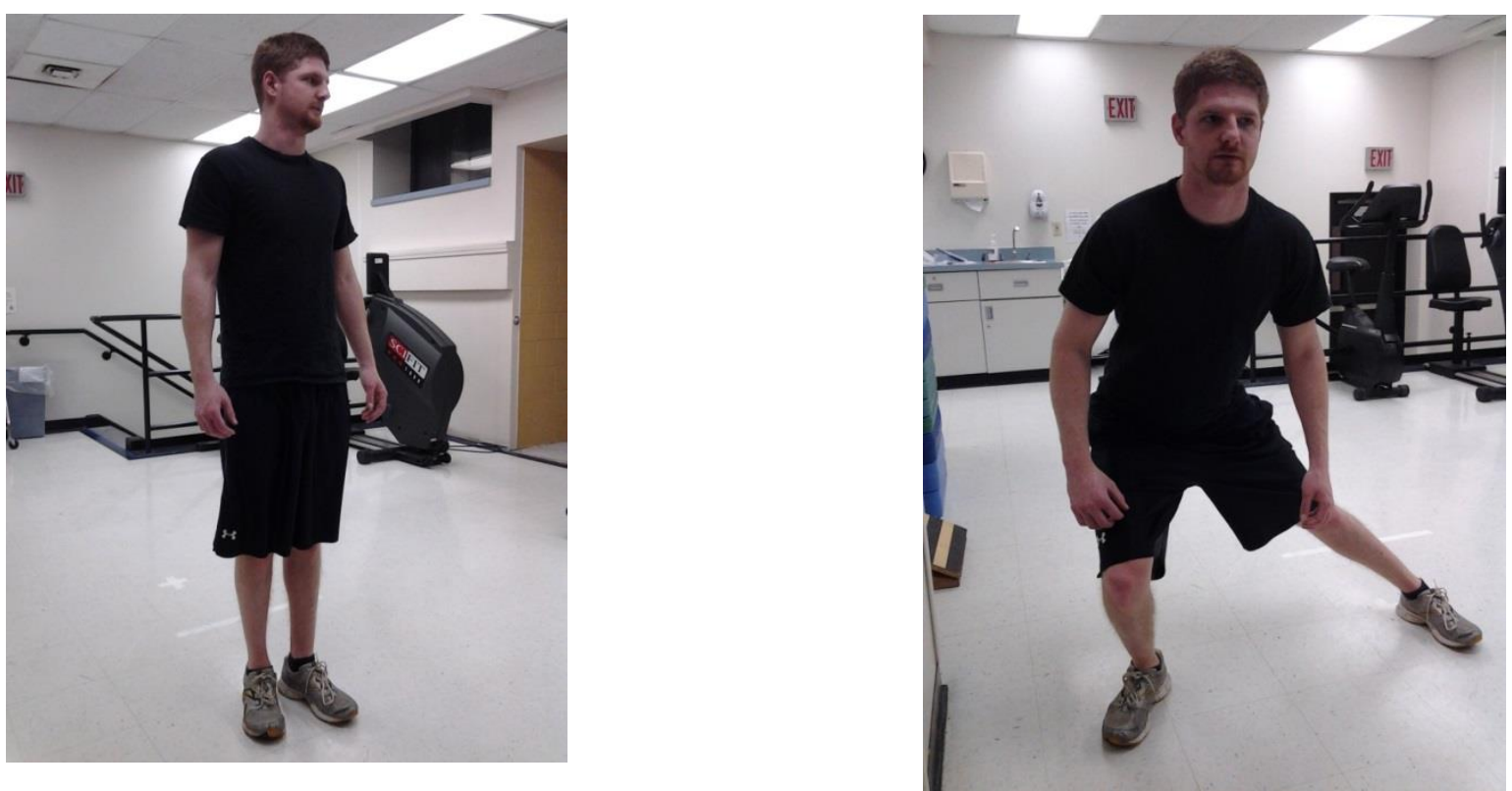
1. The subject laid supine on a table

2. The subject started with the arm across the body and the hand placed at the contralateral hip

3. The subject moved the arm into glenohumeral extension, glenohumeral abduction, glenohumeral external rotation, elbow extension, wrist extension, wrist supination, and finger extension in a smooth movement while the researcher provided submaximal resistance manually

4. The subject moved the arm into finger flexion, wrist pronation, wrist flexion, elbow flexion, glenohumeral internal rotation, glenohumeral adduction, and glenohumeral flexion in a smooth movement while the researcher provided submaximal resistance manually back to the starting posistion

5. This exercise was performed on the throwing shoulder only
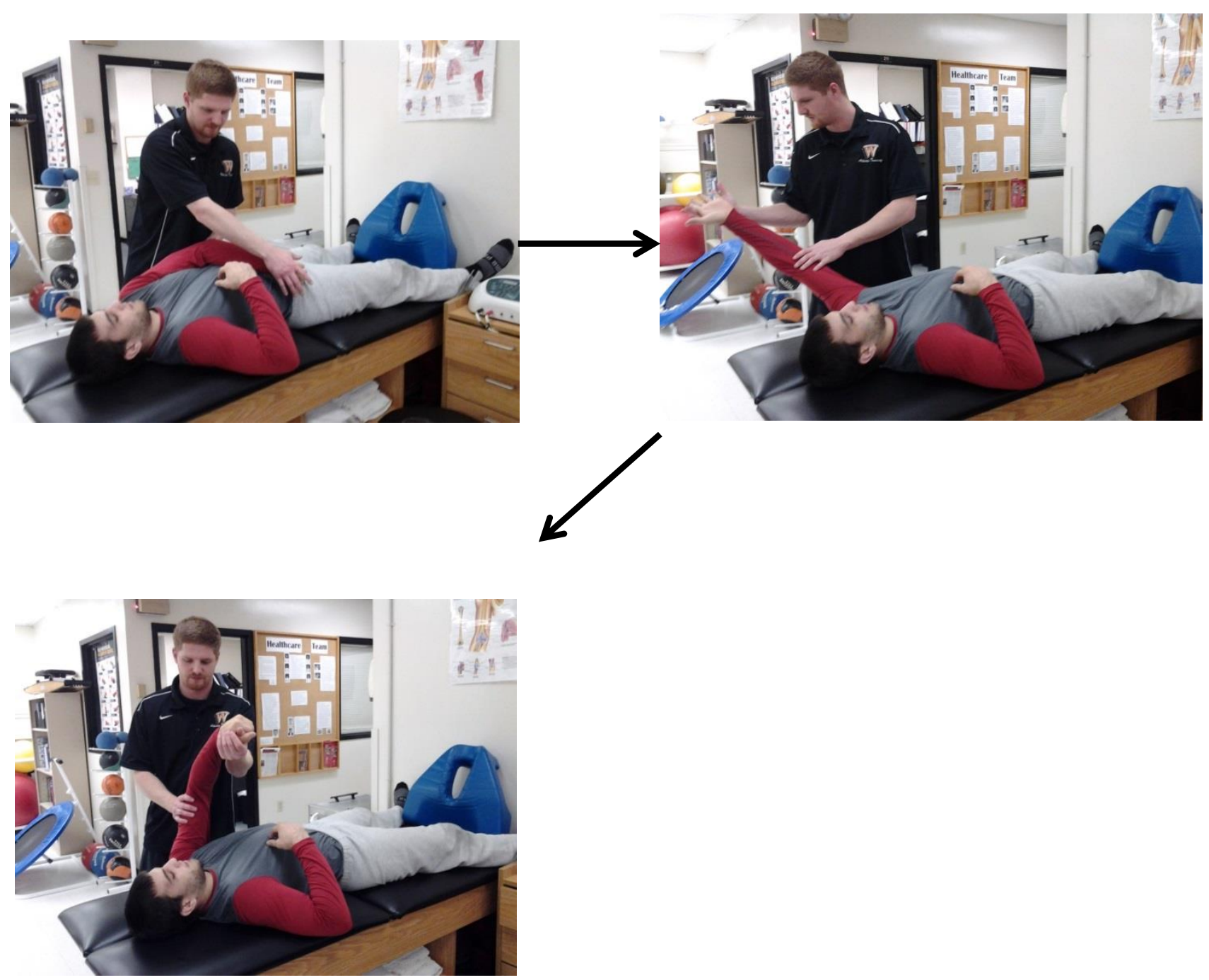


\section{$\mathrm{D}_{2}$ PFN Exercise}

1. The subject laid supine on a table.

2. The subject started with the arm across the body and the hand placed at the contralateral ear.

3. The subject moved the arm into glenohumeral extension, glenohumeral abduction, glenohumeral internal rotation, elbow extension, wrist extension, wrist pronation, and finger extension in a smooth movement while the researcher provided submaximal resistance manually.

4. The subject moved the arm into finger flexion, wrist supination, wrist flexion, elbow flexion, glenohumeral external rotation, glenohumeral adduction, and glenohumeral flexion in a smooth movement while the principal investigator provided submaximal resistance manually back to the starting position.

5. This exercise was performed on the throwing shoulder only.
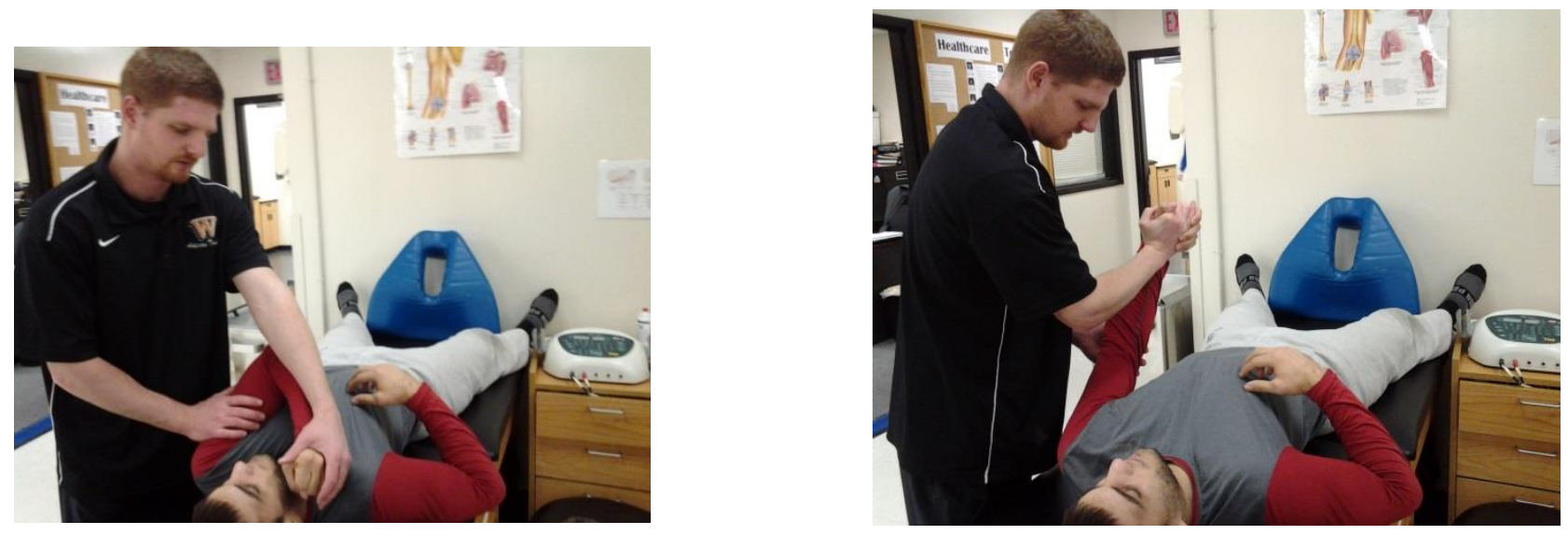

\section{Rhythmic Stabilization}

1. The subject laid supine on a table

2. The subject brought the glenohumeral joint into 90 degrees flexion and hold that position

3. The researcher tapped the subject's arm in different directions repeatedly

4. The subject tried to keep the arm in the starting position as best as they can

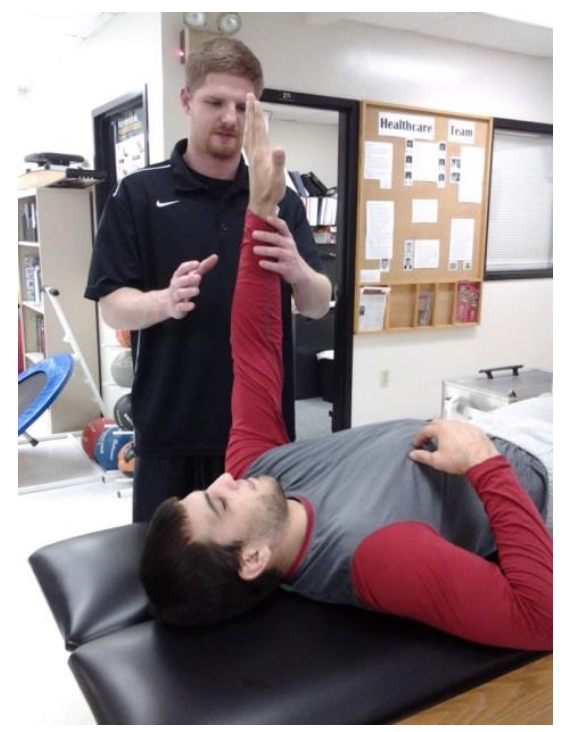


Quadruped (Double Limb Alternating)

1. Subject began on a treatment table on all fours

2. While maintaining balance, the subject raised one arm and the contralateral hip until they were in line with the torso.

3. The subject lowered the limbs to the original starting position.
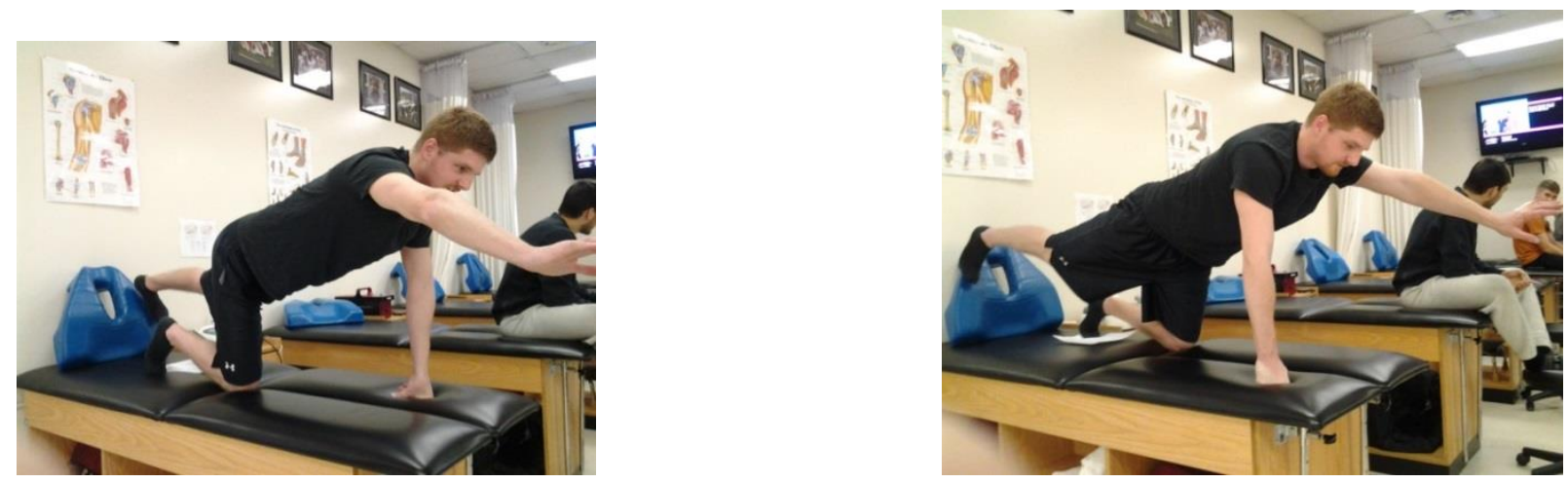

Single Leg Bridges on Airex

1. The subject laid supine on a table with the knees bent and the feet flat on an Airex pad

2. The subject extended one hip so the shoulder blades remained on the table and the torso and extended hip leg were in line. The contralateral leg as extended at the knee.

3. The subject lowered themselves back into the starting position

4. This was performed with both legs
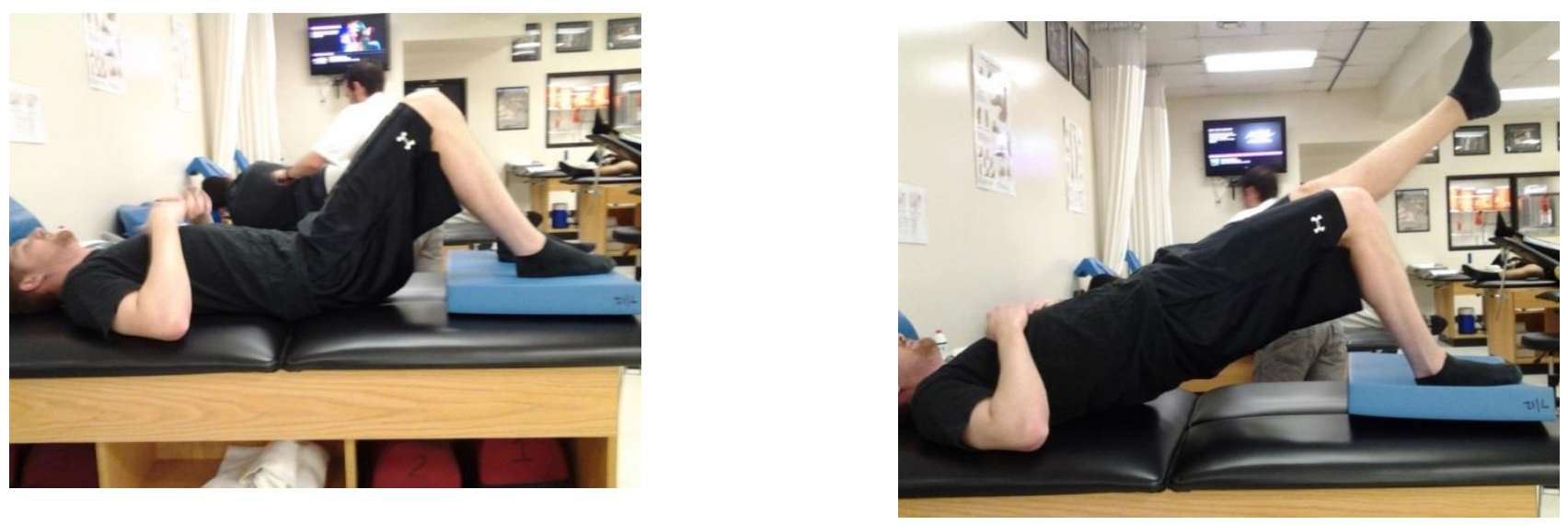


\section{Front Lunges on BOSU}

1. Subject stood upright in the middle of an empty space facing a BOSU ball

2. While maintaining an upright position of the torso, subject stepped with the stride leg into flexion onto the BOSU ball until the stance leg was parallel to the floor

3. Subject stepped back to the starting position

4. This was only performed for the stride leg
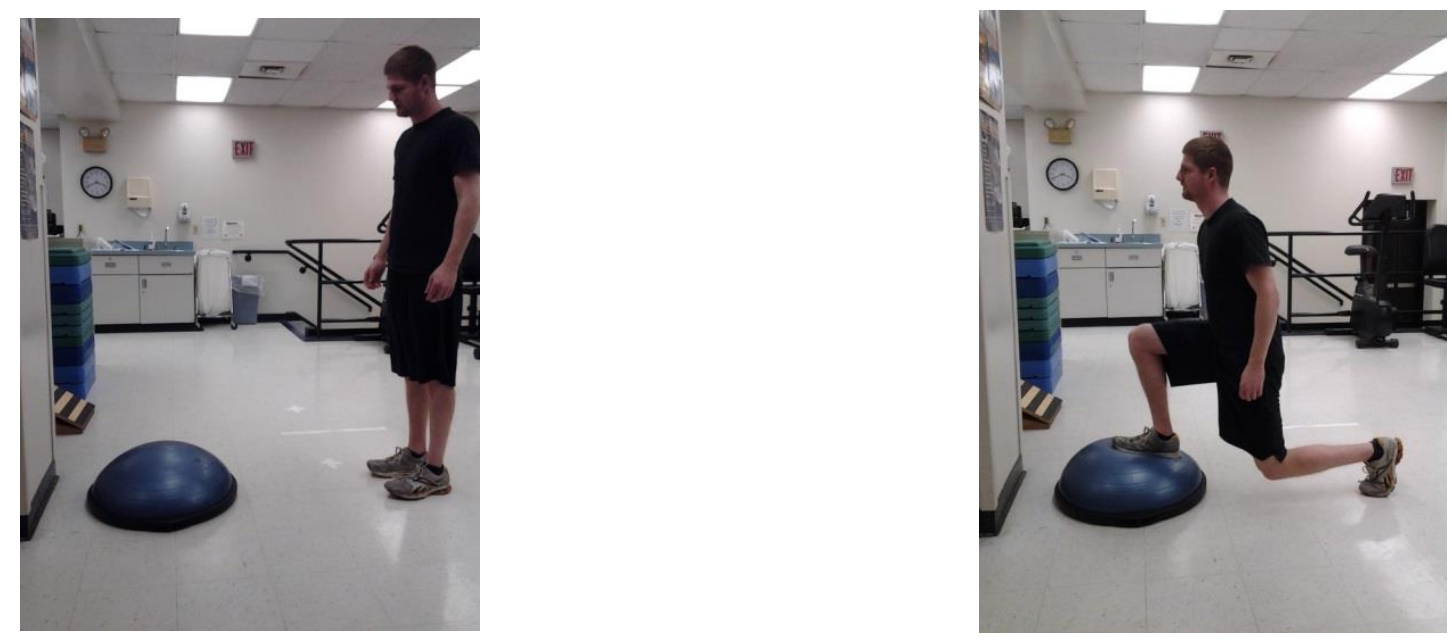

Side Lunges on BOSU

1. Subject stood upright in the middle of an empty space to the side of a BOSU ball

2. While maintaining an upright position of the torso, subject stepped into abduction with the stride leg onto a BOSU ball until the femur was parallel to the floor

3. Subject stepped back to the starting position

4. This was only performed for the stride leg
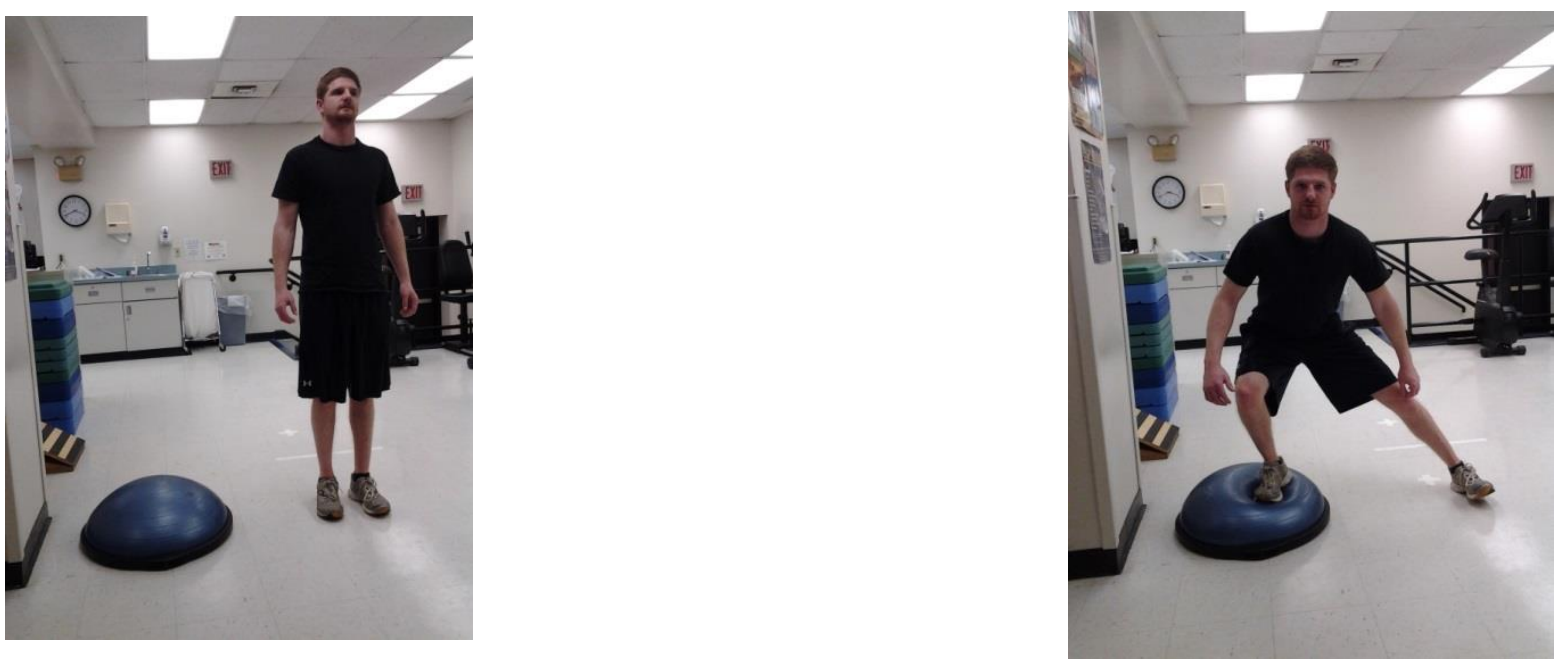


\section{BOSU Squats}

1. Subject stood with the feet firmly on a BOSU ball that was ball-side-down

2. The subject performed a squat until the femurs were parallel to the floor while they maintained an upright torso

3. Subject returned to the starting position
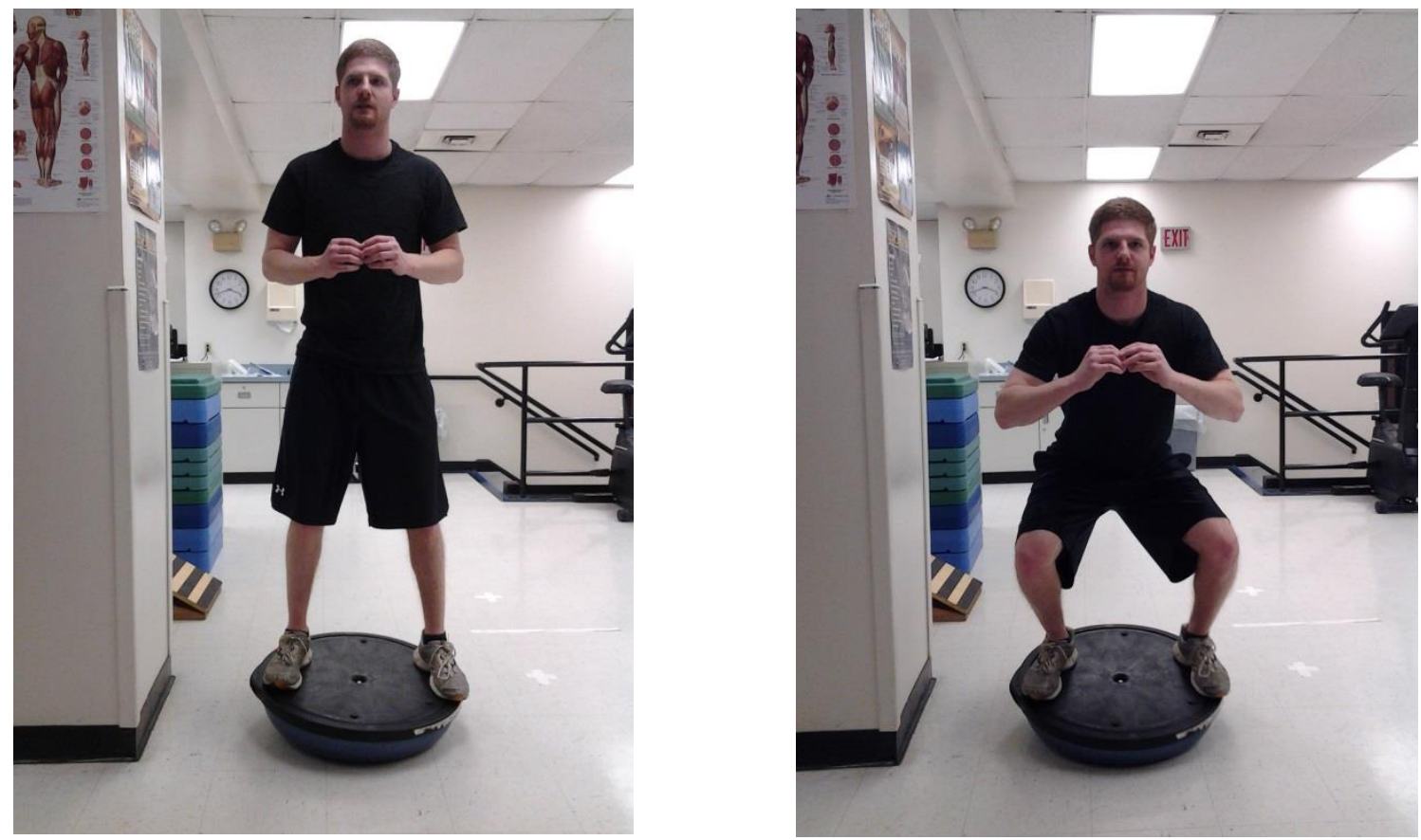
BOSU Push Ups

1. Subject assumed a push up position on a BOSU ball that was ball-side-down

2. Subject lowered the body while maintaining proper form

3. Subject raised the body up to the starting position
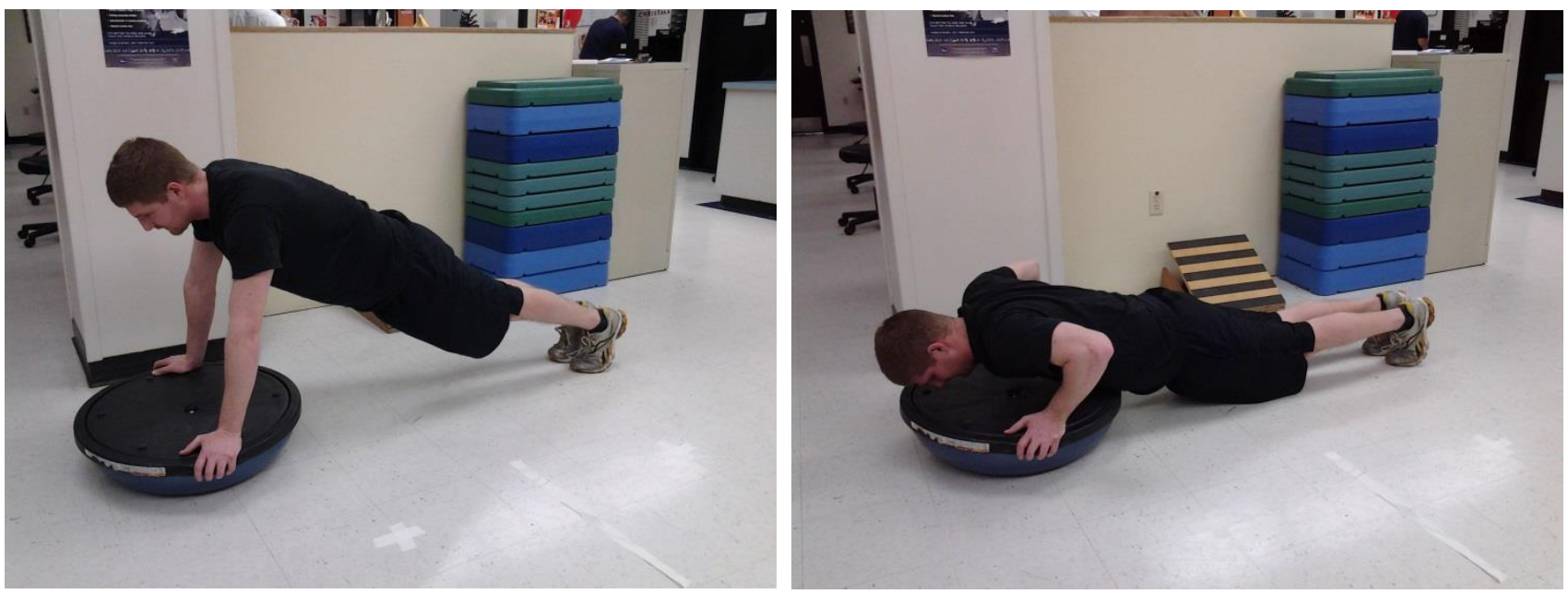

Physioball Push Up Balance

1. Subject was prone on a table with the upper body cantilevered off the table.

2. While maintaining shoulder flexion at 90 degrees and keeping the elbows extended, subject balanced the upper body on a physioball

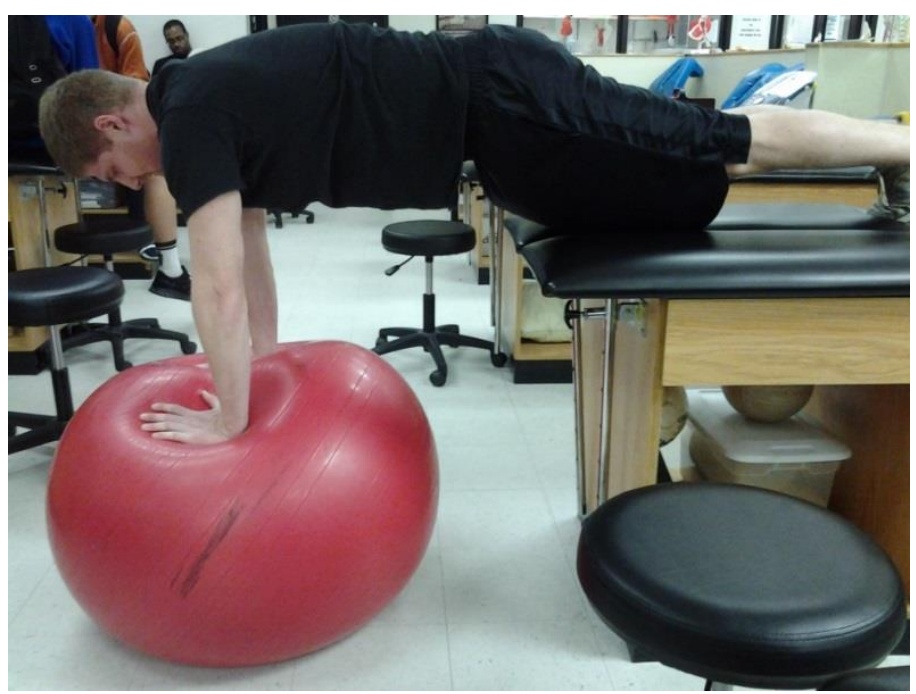




\section{Leg Lift with Rotation}

1. Subject laid supine on the table with the lower extremity cantilevered off.

2. While gripping the table with his or her hands, subject raised the legs up to 90 degrees flexion

3. Subject rotated the hips to the side so the legs were at a 45 degree angle in relation to the table

4. Subject rotated the hips to the opposite side so the legs were at in the same position as they were on the contralateral side

5. Subject returned to the starting position so the body was in alignment.
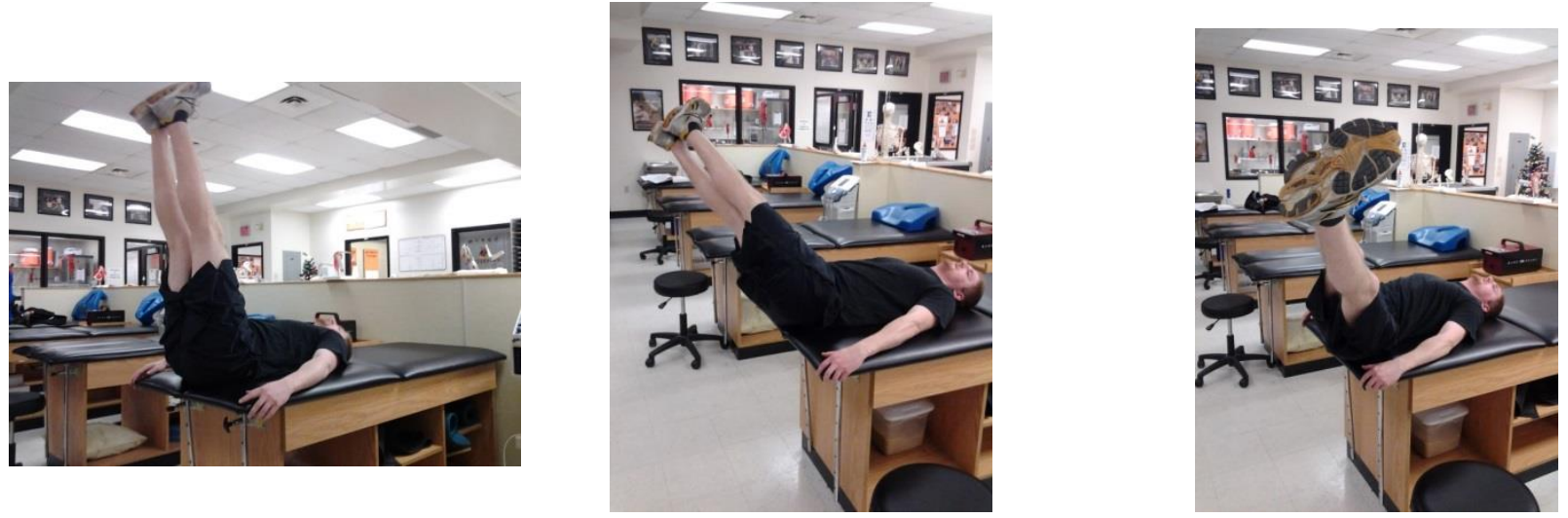

Double Leg Bridges on Physioball

1. Subject laid supine on a table with the legs extended and heels together on a physioball

2. Subject extended the hips so the body was in line with itself

3. Subject held this position for 5 seconds

4. Subject lowered the hips to the starting position
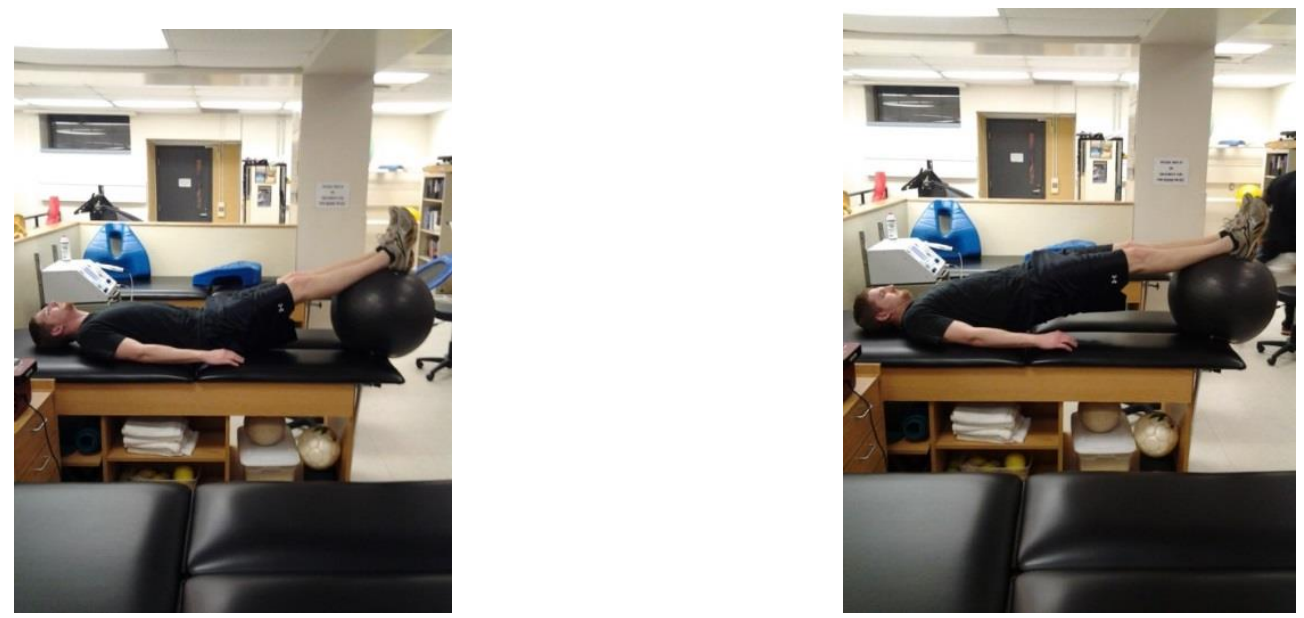
Medicine Ball Lateral Swing Hop

1. Subject stood in the middle of an empty space holding a medicine ball

2. Subject placed the medicine ball about one hip

3. The subject simultaneously swung the medicine ball laterally toward the opposite hip while also hopping laterally to the other leg

4. The subject repeated the motion and hopped to the starting leg
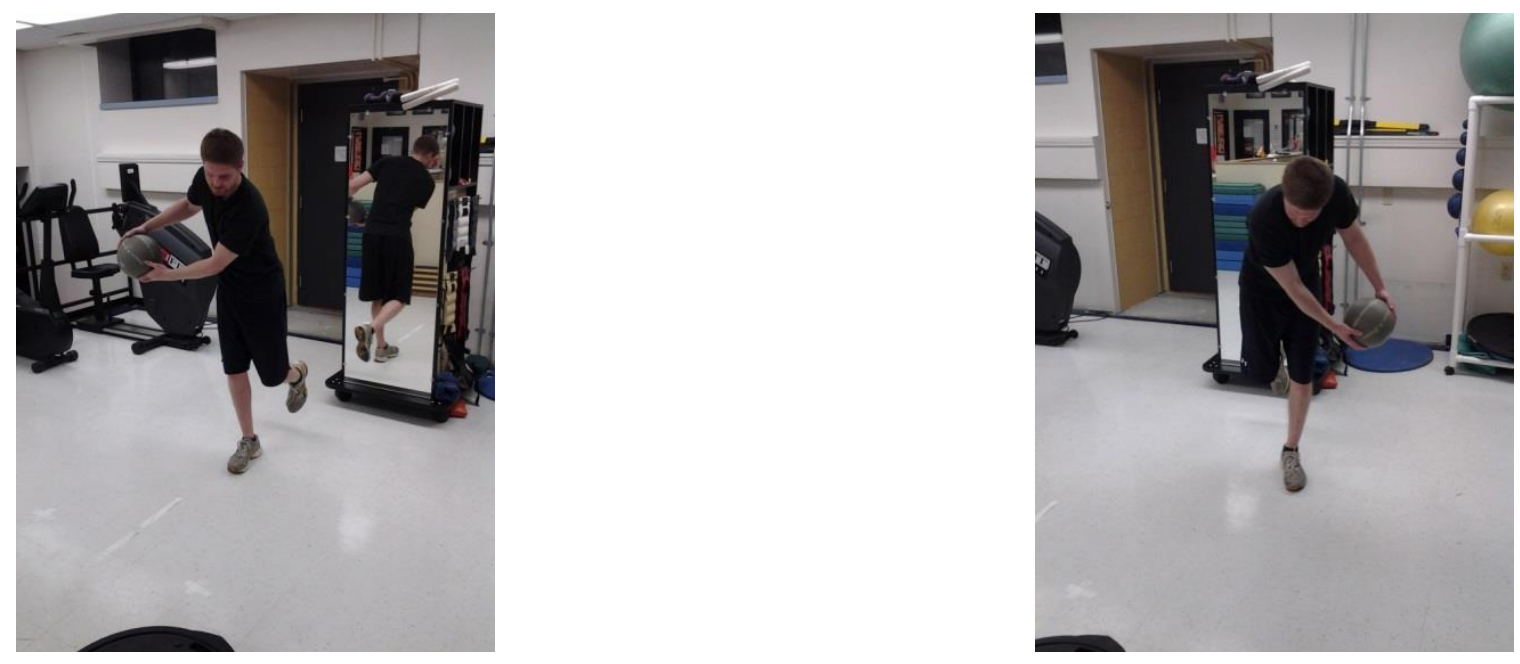

\section{Lateral Ground Hop}

1. The subject began in a kneeling position with opposite arm and knee orientation. The stance leg during the throwing motion was the lead leg.

2. The subject initiated a quick and powerful hip extension, hip abduction, and knee extension movement to propel them to a single leg standing position on the contralateral leg

3. The subject knelt back to the starting position
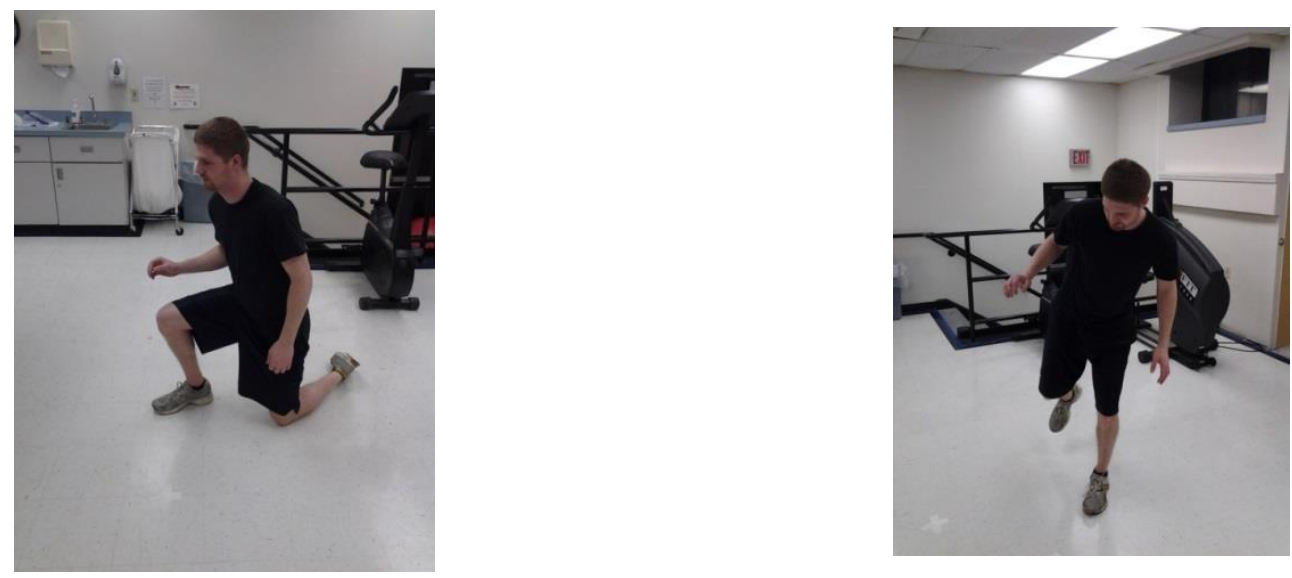
Kneeling 90/90 Forward Ball Toss

1. Subject was in a kneeling position with the lead leg being contralateral to the throwing arm and be facing a rebounder 10 feet away.

2. Subject positioned the throwing arm to 90 degrees abduction, 90 degrees external rotation, and elbow flexed to 90 degrees

3. With a quick, explosive movement, subject internally rotated the arm and threw a ball against a trampoline to rebound the ball back to the subject

4. Subject caught the ball and returned to the starting position

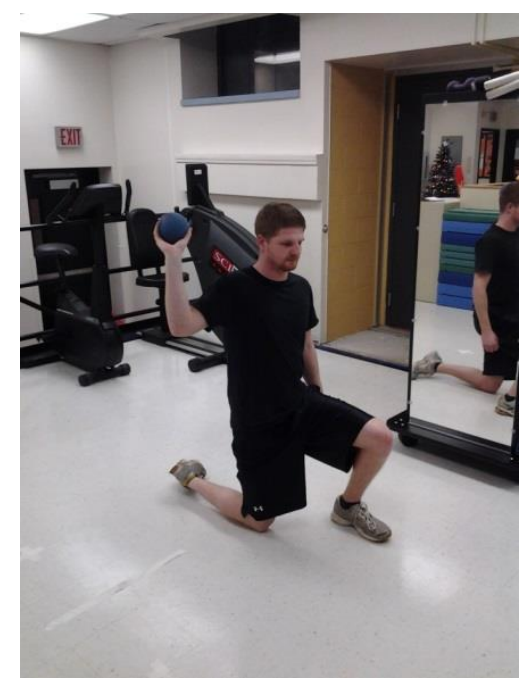

Kneeling 90/90 Reverse Toss

1. Subject was in a kneeling position with the lead leg as the contralateral to the throwing arm and be facing away from a rebounder 10 feet away

2. Subject positioned the throwing arm to 90 degrees abduction, 90 degrees external rotation, and elbow flexed to 90 degrees. The subject was allowed to turn the head to look at where they were throwing

3. With a quick, explosive movement, the subject externally rotated the arm and threw a ball against a trampoline that rebounded the ball back to the subject

4. Subject caught the ball and returned to the starting position

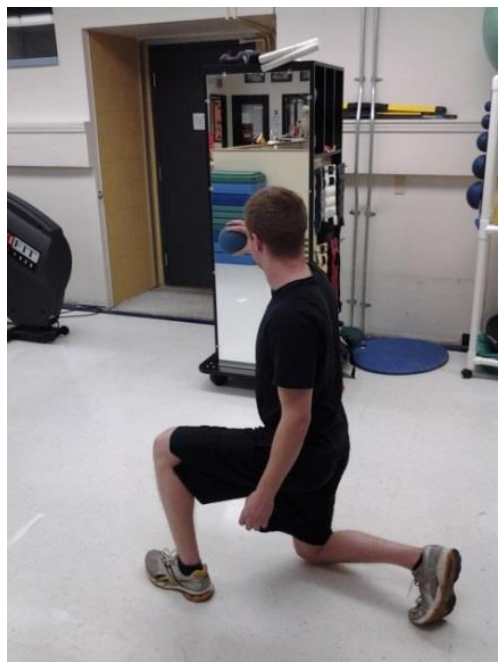




\section{Kneeling Chop}

1. Subject resumed a kneeling position with the trunk erect. The lead leg was the contralateral leg to the throwing shoulder

2. The subject held al meter exercise bar that was attached to a thera-band. The exercise bar as placed diagonally in the two o'clock position. The bottom hand was the ipsilateral arm to the lead leg and grasped the bar at the bottom

3. The top hand grasped the bar at the midpoint

4. The subject flexed and rotated the trunk toward the lead leg while pulling with the bottom hand and pushing with the top hand to perform a chopping motion.

5. The subject returned to the starting position
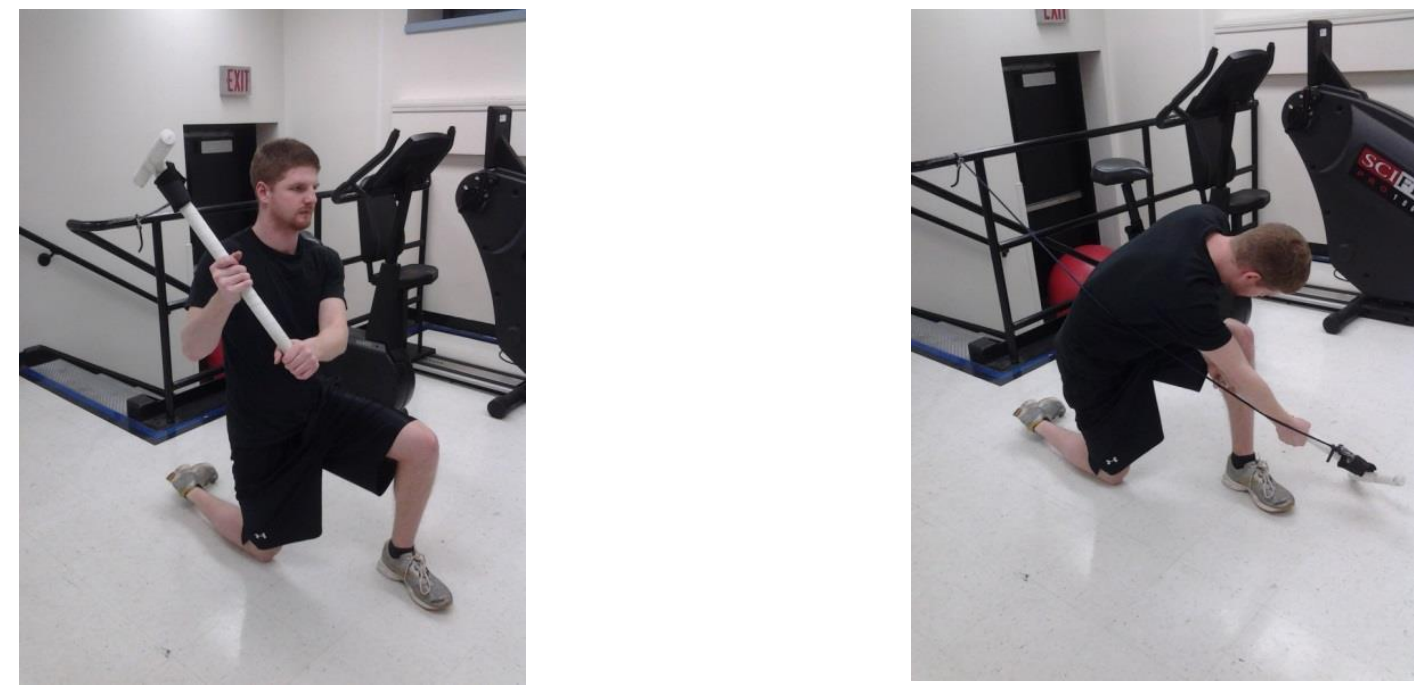

Medicine Ball Rebound Lateral Toss

1. The subject stood perpendicularly 5 feet away from a wall while holding a medicine ball with both hands down waist level.

2. The subject rotated the core toward the wall and used the force generated by the core to toss the ball against the wall

3. The subject caught the medicine ball and returned to the starting position

4. This was repeated for the opposite side.
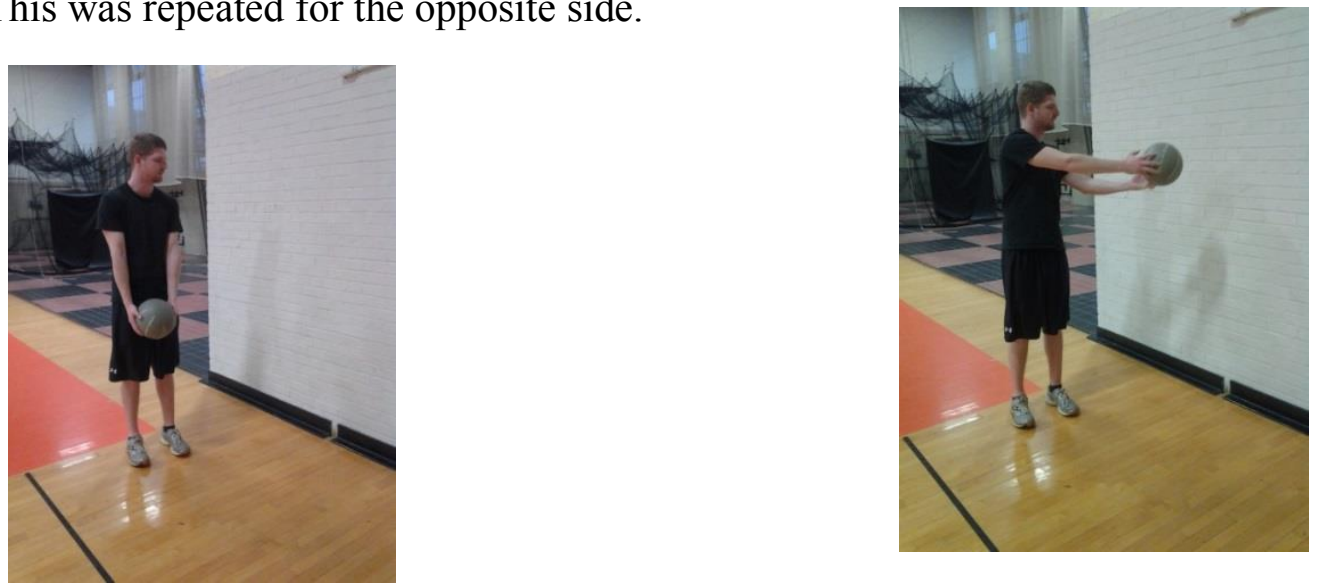


\section{Front Drop Back Lunge}

1. The athlete stood in an open space with a medicine ball under the chin

2. The subject brought the stride leg into hip extension, and adduction behind the stance leg until the stance leg femur was parallel to the ground

3. The subject returned to the starting position
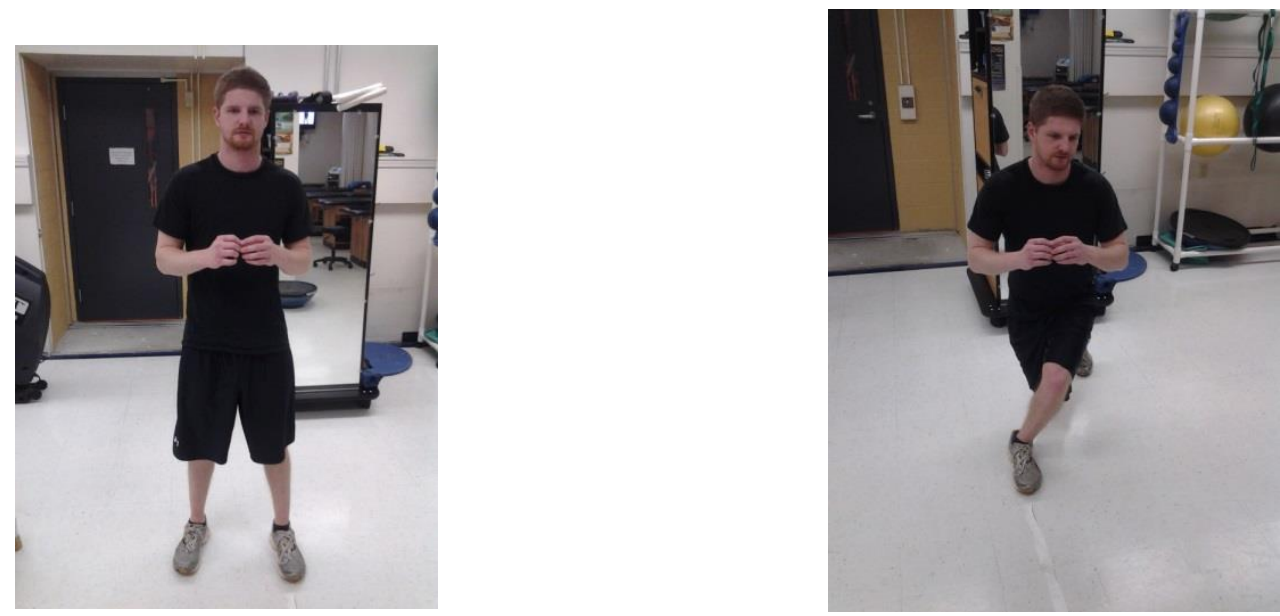

Plyometric Lateral Ground Hop

1. The subject began in a kneeling position with opposite arm and knee orientation next to a 6 " plyo-box. The stance leg during the throwing motion was the lead leg.

2. The subject initiated quick and powerful hip extension, hip abduction, and knee extension movement that propeled them onto a 6" plyo-box into a single leg standing position on the contralateral leg

3. The subject knelt back to the starting position
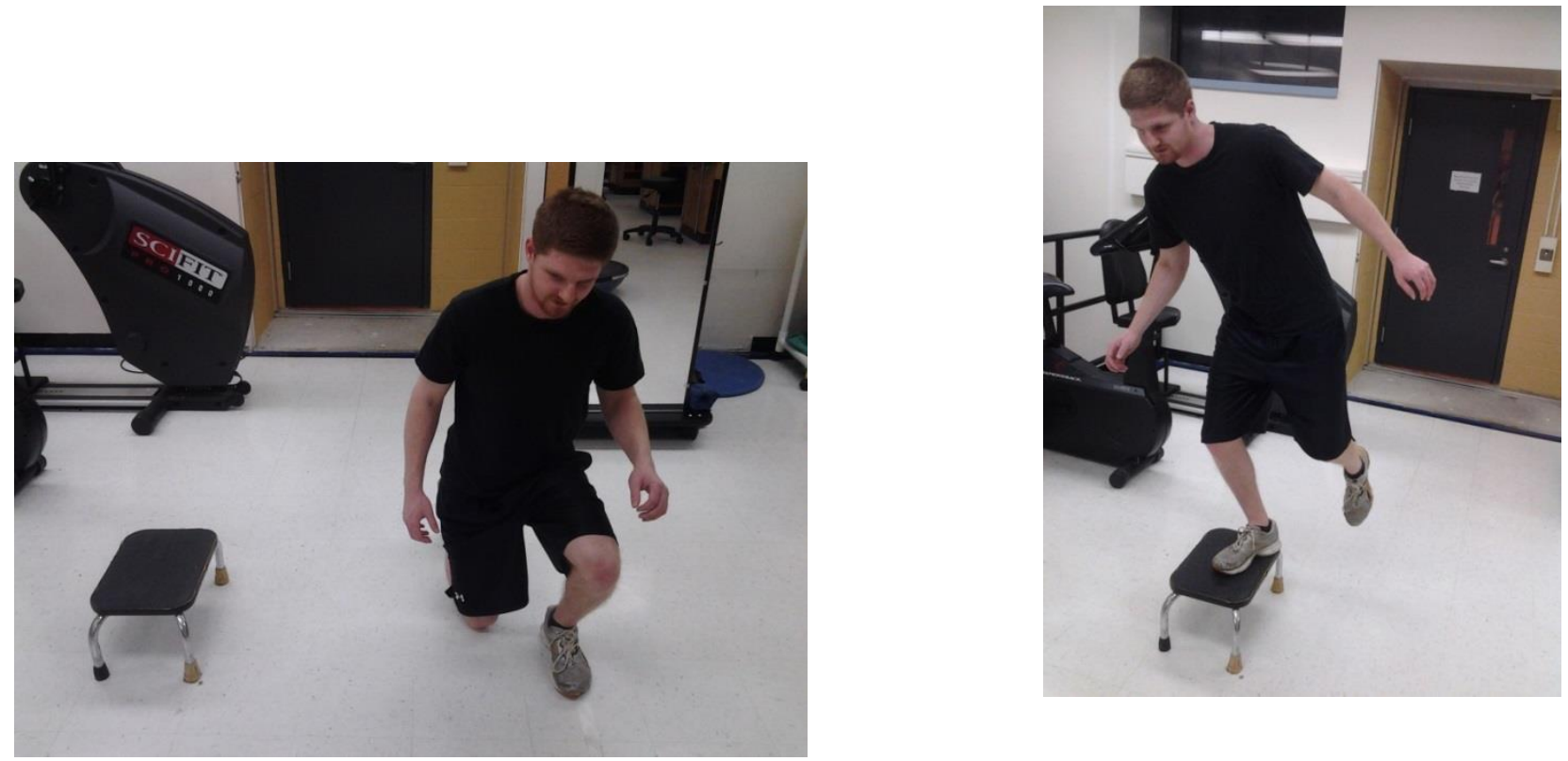
Lunge Punches

1. Subject stood facing away from a wall with the arm abducted to 90 degrees, neutral rotation, and elbow flexed to 90 degrees

2. Subject gripped a resistance band with the throwing arm that was fixed to the wall at shoulder height

3. Subject performed a forward lung with the contralateral leg and extended the elbow and protracted the scapula, performing a punching motion

4. The subject retracted the scapula, flexed the elbow, and stepped back to return to the starting position
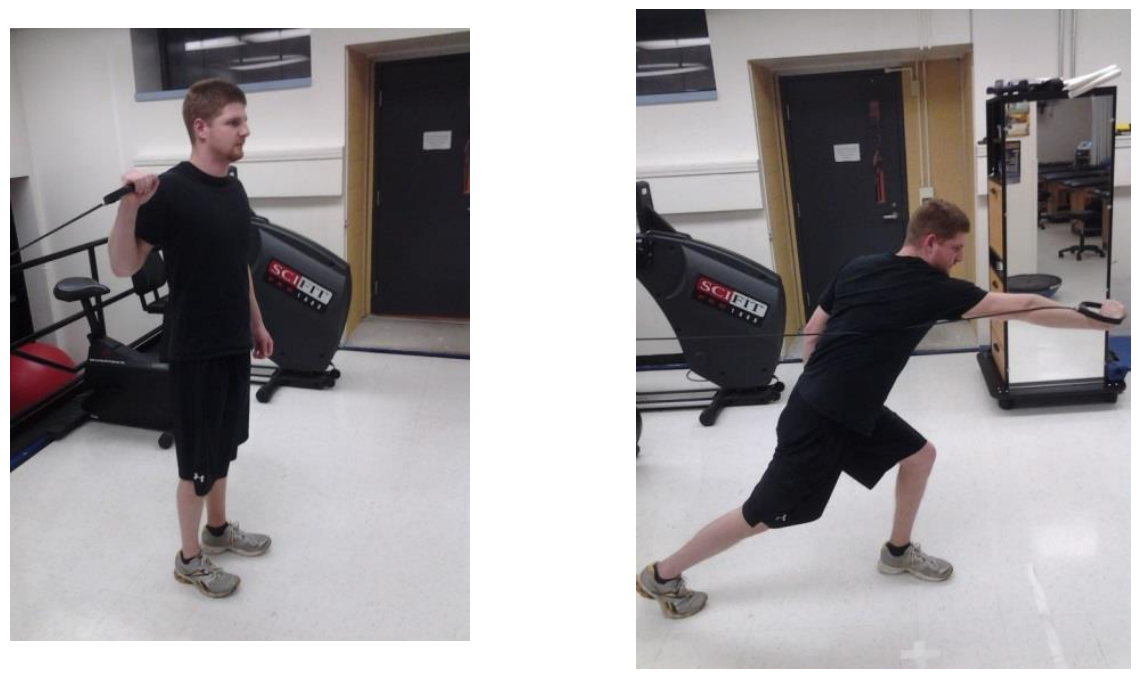

\section{Lawnmower Pulls}

1. The subject stood facing a wall with a resistance band attached at ground level. The stride leg was directly in front of the attached resistance band and the stance leg was extended slightly behind them. The knees were slightly bent

2. The subject grasped the resistance band with the throwing shoulder.

3. In a smooth motion, the subject extended the trunk, retracted the scapula, abducted the shoulder, horizontally abducted the shoulder, and rotated the trunk away from the resistance

4. In a slow and controlled manner the subject returned back to the starting position
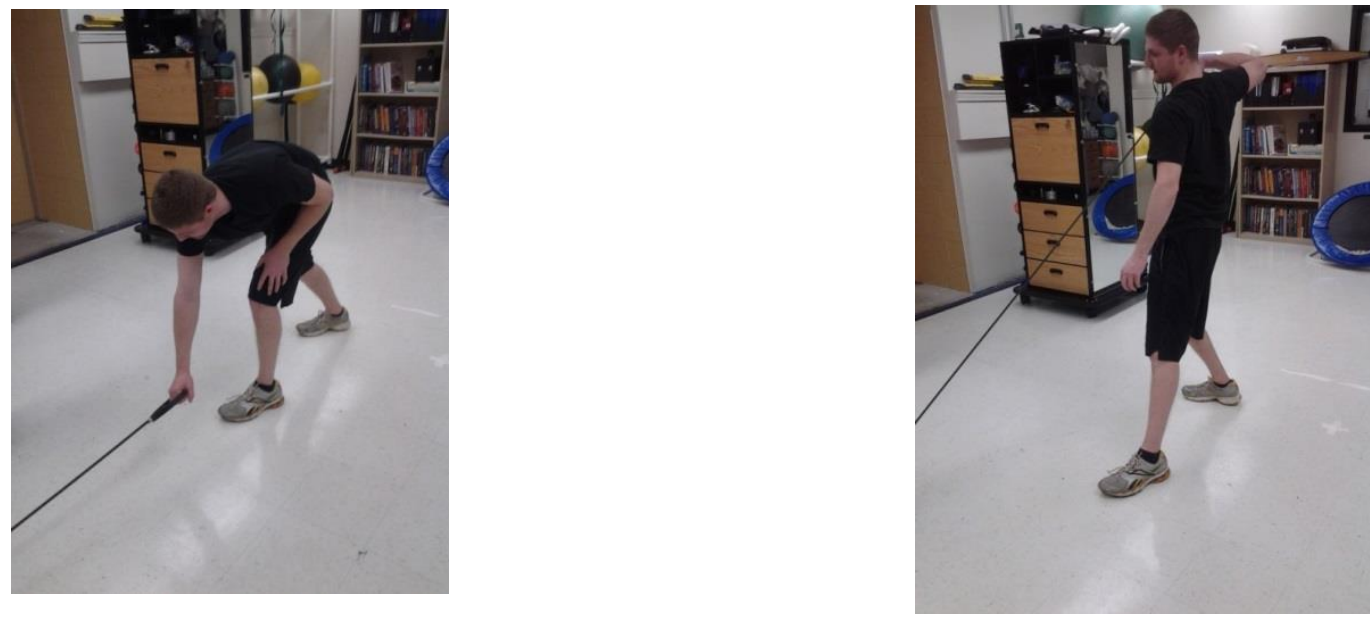


\section{Stance Balance}

1. The subject stood perpendicular to a wall with a resistance band attached to it at waist height. The legs were spread 36 inches apart. The back leg was the stance leg. The other end of the resistance band went around the subject's waist to tension

2. The subject adducted the stance leg and flexed the stride leg's hip and knee.

3. This position was held for 5 seconds

4. In a slow and controlled manner, subject returned to the starting position

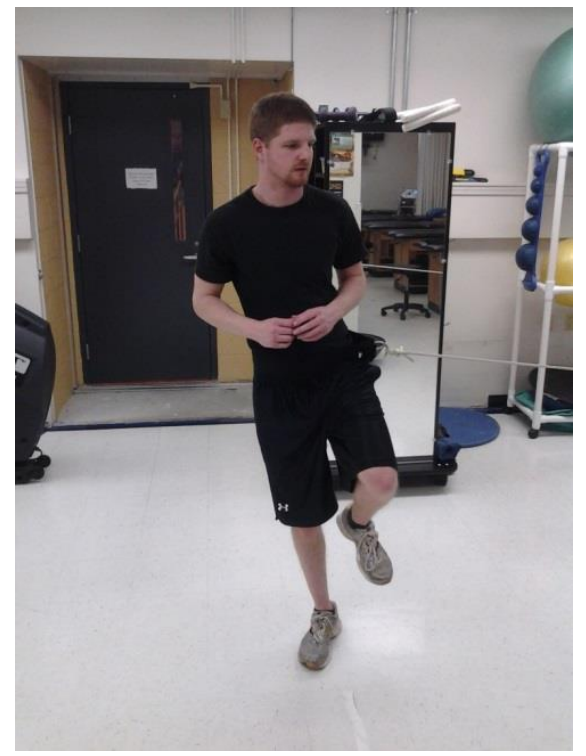


Life/Place with Dumbbell

1. Subject started in a kneeling position. The lead leg was the stride leg. A dumbbell was placed directly in front of the lead leg. The subject started with a flexed trunk and with the throwing shoulder grasping the dumbbell.

2. The subject extended the lead hip and lead knee, extended the trunk, rotated the trunk away from the lead leg, retracted the scapula and flexed the shoulder so that the subject was standing straight up with the arm straight above them holding the weight.

3. The subject lunged with the lead leg, flexed the trunk, and lowered the weight back down and placed it back in front of the lead leg.
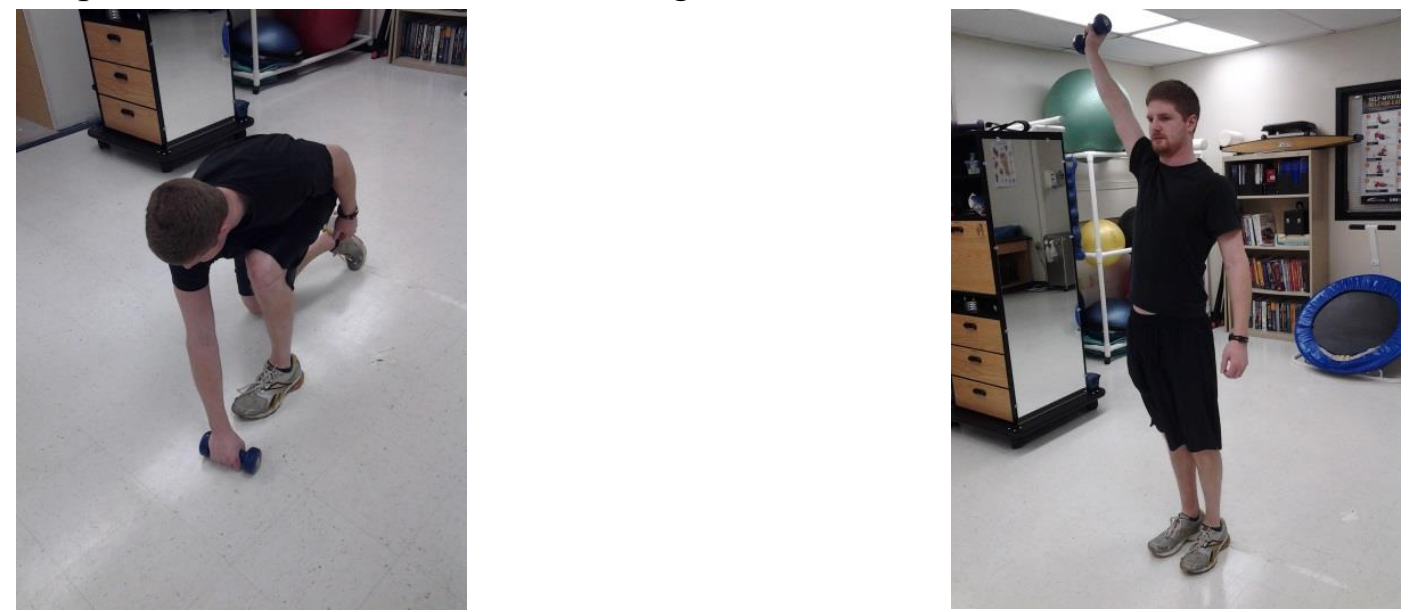

\section{Standing Chop}

1. Subject stood with the trunk erect.

2. The subject held a1 meter exercise bar that was attached to a thera-band. The exercise bar was placed diagonally in the two o'clock position. The bottom hand was the ipsilateral arm to the lead leg and it grasped the bar at the bottom

3. The top hand grasped the bar at its midpoint

4. The subject flexed and rotated the trunk toward the lead leg, pulled down with the bottom hand, and pushed with the top hand to perform a chopping motion.

5. The hips and legs did not move and served to stabilize the subject

6. The subject returned to the starting position in a slow and controlled manner
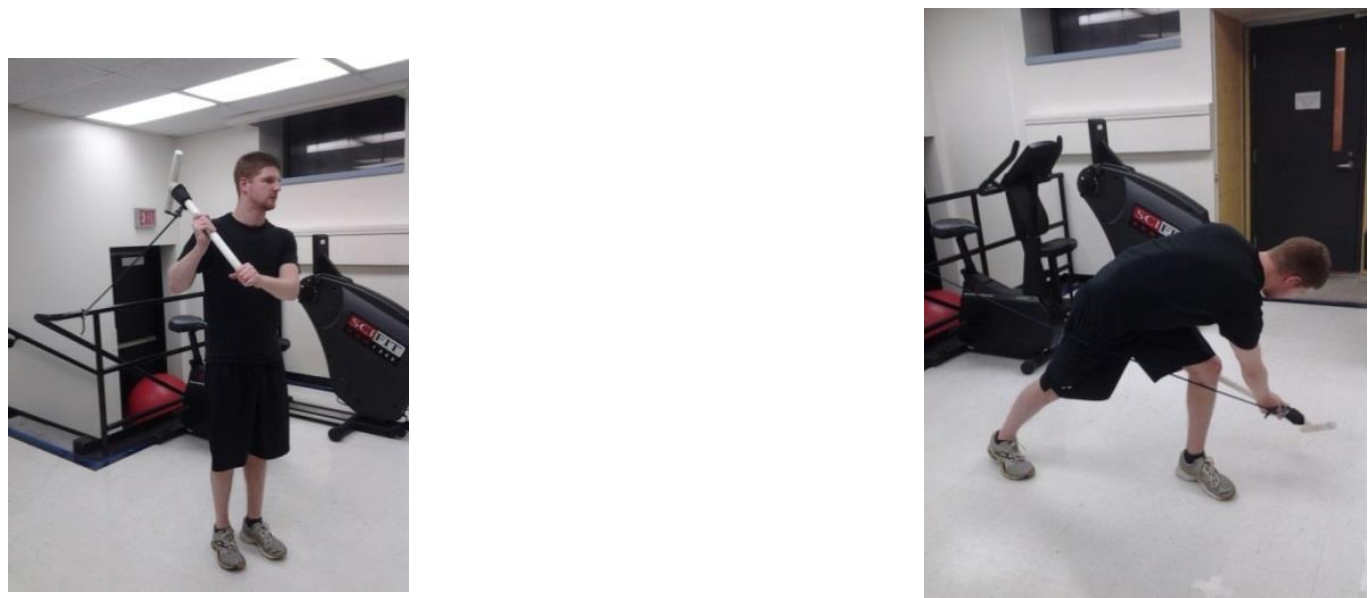


\section{Thera-band Throw with Rotation}

1. The subject began standing perpendicular to a wall with a resistance band fixed to the wall. The stance leg was closest to the wall.

2. The subject grasped the other end of the resistance band with the throwing hand. The arm was at 90 degrees abduction and full external rotation

3. The subject strided and lunged with the contralateral leg

4. The subject contralaterally rotated the trunk, then internally rotated, extended, and adducted the shoulder, and extended the elbow, mimicking the throwing motion

5. The subject returned to the starting position in a slow and controlled manner
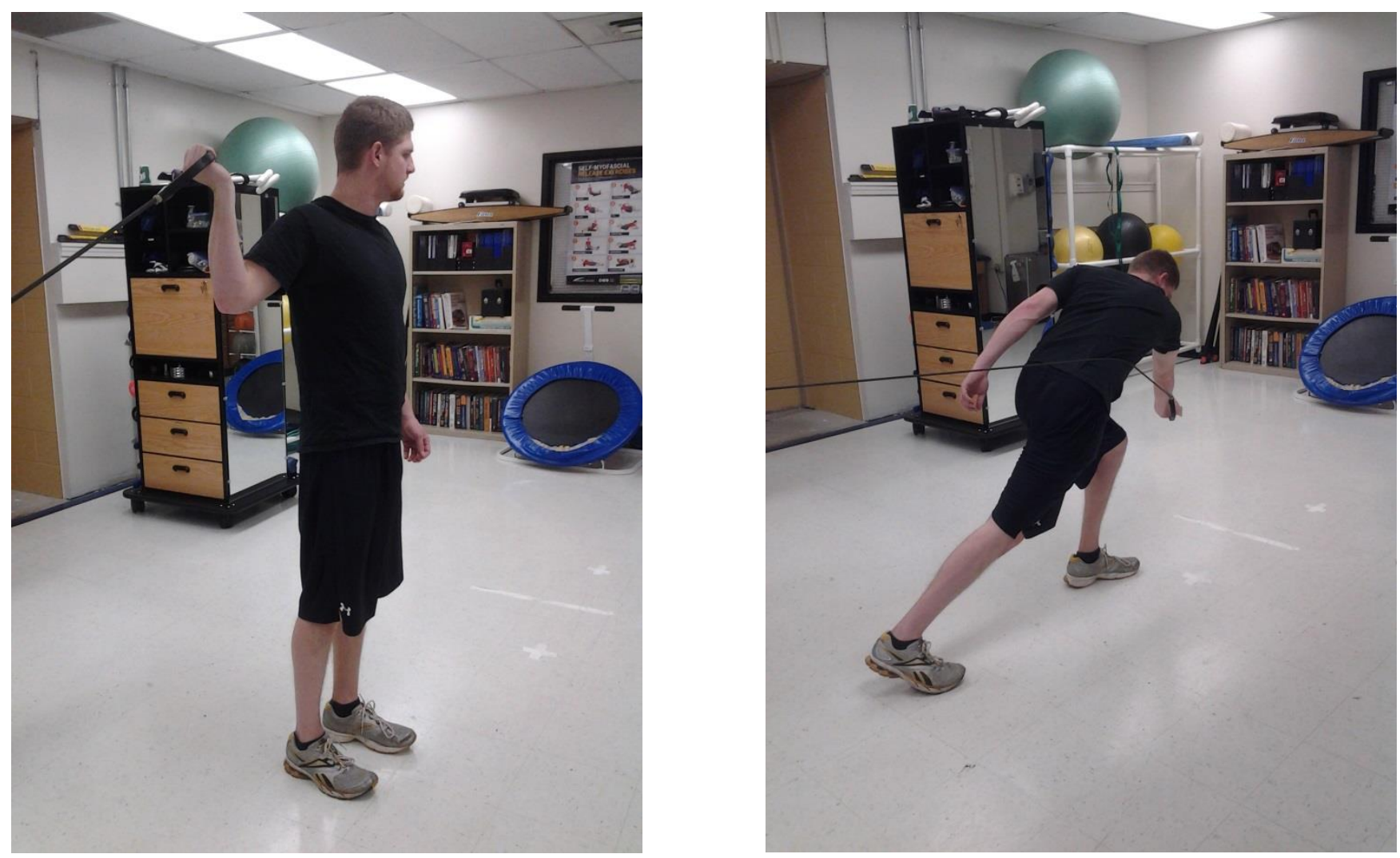


\section{APPENDIX D}

\section{ADDITIONAL RESULTS}

Table D1. Descriptive Statistics of Means and Standard Deviations of All Dependent Variables

\begin{tabular}{|c|c|c|c|c|c|c|c|c|c|c|}
\hline Test & Co & & & & Experi & nental & & & & \\
\hline & Pre-Test & Post-Test & & & Pre-Test & Post-Test & & & & \\
\hline & Mean & Mean & $\mathrm{ES}_{\text {Time }}$ & $\mathrm{CI}_{\text {Time }}$ & Mean & Mean & $\mathrm{ES}_{\text {Time }}$ & $\mathrm{CI}_{\text {Time }}$ & $\mathrm{ES}_{\text {Group }}$ & $\mathrm{CI}_{\text {Group }}$ \\
\hline JPS $_{\text {Right }}$ & $7.48 \pm 6.29$ & $8.96 \pm 4.29$ & 0.24 & $(-0.69,1.16)$ & $5.99 \pm 4.59$ & $5.20 \pm 3.35$ & -0.17 & $(-1.05,0.71)$ & -0.88 & $(-1.82,0.07)$ \\
\hline $\mathrm{JPS}_{\text {Left }}$ & $4.74 \pm 3.18$ & $6.11 \pm 3.58$ & 0.43 & $(-0.50,1.37)$ & $5.83 \pm 4.41$ & $7.00 \pm 4.21$ & 0.27 & $(-0.62,1.15)$ & 0.25 & $(-0.66,1.15)$ \\
\hline FTPI & $2.00 \pm 0.55$ & $2.06 \pm 0.52$ & 0.11 & $(-0.82,1.03)$ & $1.76 \pm 0.30$ & $1.53 \pm 0.44$ & -0.77 & $(-1.67,0.14)$ & -1.02 & $(-1.98,-0.06$ \\
\hline Velocity & $50.56 \pm 6.82$ & $49.89 \pm 5.79$ & -0.10 & $(-1.02,0.83)$ & $50.13 \pm 3.68$ & $\begin{array}{c}52.26 \pm \\
3.99\end{array}$ & 0.58 & $(-0.32,1.47)$ & 0.41 & $(-0.50,1.32)$ \\
\hline CKCUEST & $0.25 \pm 0.06$ & $0.28 \pm 0.07$ & 0.50 & $(-0.44,1.44)$ & $0.25 \pm 0.04$ & $0.28 \pm 0.06$ & 0.75 & $(-0.16,1.66)$ & 0 & $(-0.90,0.90)$ \\
\hline
\end{tabular}

Key: JPS $\mathrm{Right}_{\mathrm{R}}=$ Joint Position Sense Error for the right arm, $\mathrm{JPS}_{\mathrm{Left}}=$ Joint position sense error for the left arm, FTPI=Functional Throwing Performance Index Score, CKCUEST=Closed Kinetic chain upper extremity stability test, $\mathrm{ES}_{\text {Time }}=$ Effect size for time, $\mathrm{ES}_{\mathrm{Group}}=\mathrm{Effect}_{\mathrm{size}}$ for group, $\mathrm{CI}_{\mathrm{Time}}=$ Confidence interval for time, $\mathrm{CI}_{\text {Group }}=$ Confidence interval for group

**All subjects were similar at baseline or all dependent variables 
Table D2. Minimal detectable change values

\begin{tabular}{lccc}
\hline Measure & MDC Value & $\Delta \underline{\text { Control }}$ & $\Delta \underline{\underline{\text { Experimental }}}$ \\
\cline { 2 - 3 } Right rotation error & -5.72 & 1.4822 & -0.8 \\
Left rotation error & -4.45 & 1.37 & 1.16 \\
FTPI & -0.313 & 0.05 & 0.229 \\
Throwing Velocity & 1.95 & -0.67 & 2.13 \\
CKCUEST & 0.021 & 0.026 & 0.030 \\
\hline
\end{tabular}

Key: $\mathrm{MDC}=$ Minimal detectable change, FTPI $=$ Functional throwing performance index, CKCUEST $=$ Closed kinetic chain upper extremity stability test, $\underline{\Delta}$ Control $=$ Pre/posttest difference for control group, $\underline{\Delta}$ Experimental $=$ Pre/posttest difference for experimental group

Table D3. Pairwise Comparison for Pre-Test and Post-Test

\begin{tabular}{lc}
\hline Test & $\underline{\mathrm{P}}$ \\
Right Rotational Error & 0.802 \\
Left Rotational Error & 0.286 \\
FTPI & 0.533 \\
Throwing Velocity & 0.022 \\
CKCUEST & 0.002 \\
\hline
\end{tabular}

Key: FTPI=Functional throwing performance index, CKCUEST $=$ Closed kinetic chain upper extremity stability test, $\mathrm{P}=\mathrm{p}$-value

Table D4. Pairwise Comparison for Experimental Group and Control Group

$\begin{array}{lc}\text { Test } & \underline{\mathrm{P}} \\ \text { Right Rotational Error } & 0.141 \\ \text { Left Rotational Error } & 0.480 \\ \text { FTPI } & 0.033 \\ \text { Throwing Velocity } & 0.671 \\ \text { CKCUEST } & 0.814\end{array}$

Key: FTPI=Functional throwing performance index, CKCUEST $=$ Closed kinetic chain upper extremity stability test, $\mathrm{P}=\mathrm{p}$-value 
Figure D1. Left arm joint position sense plot

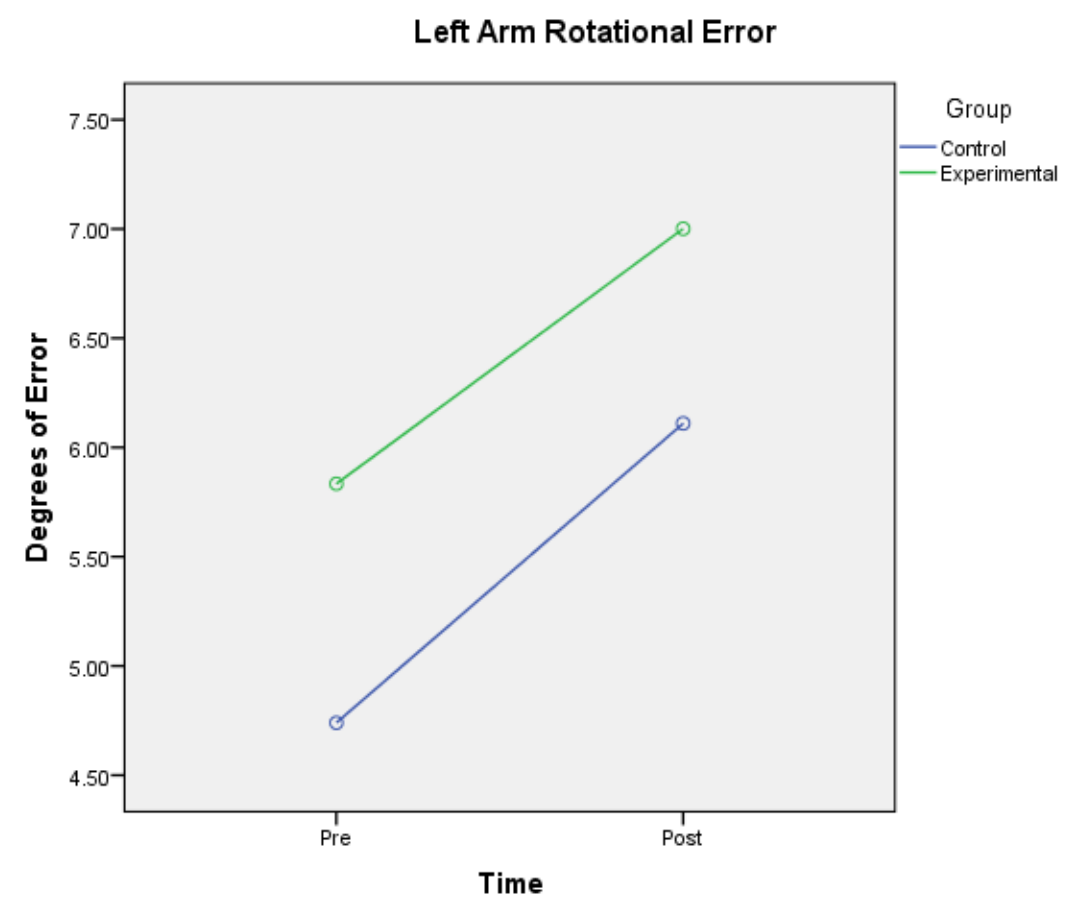

Figure D2. Right arm joint position sense plot

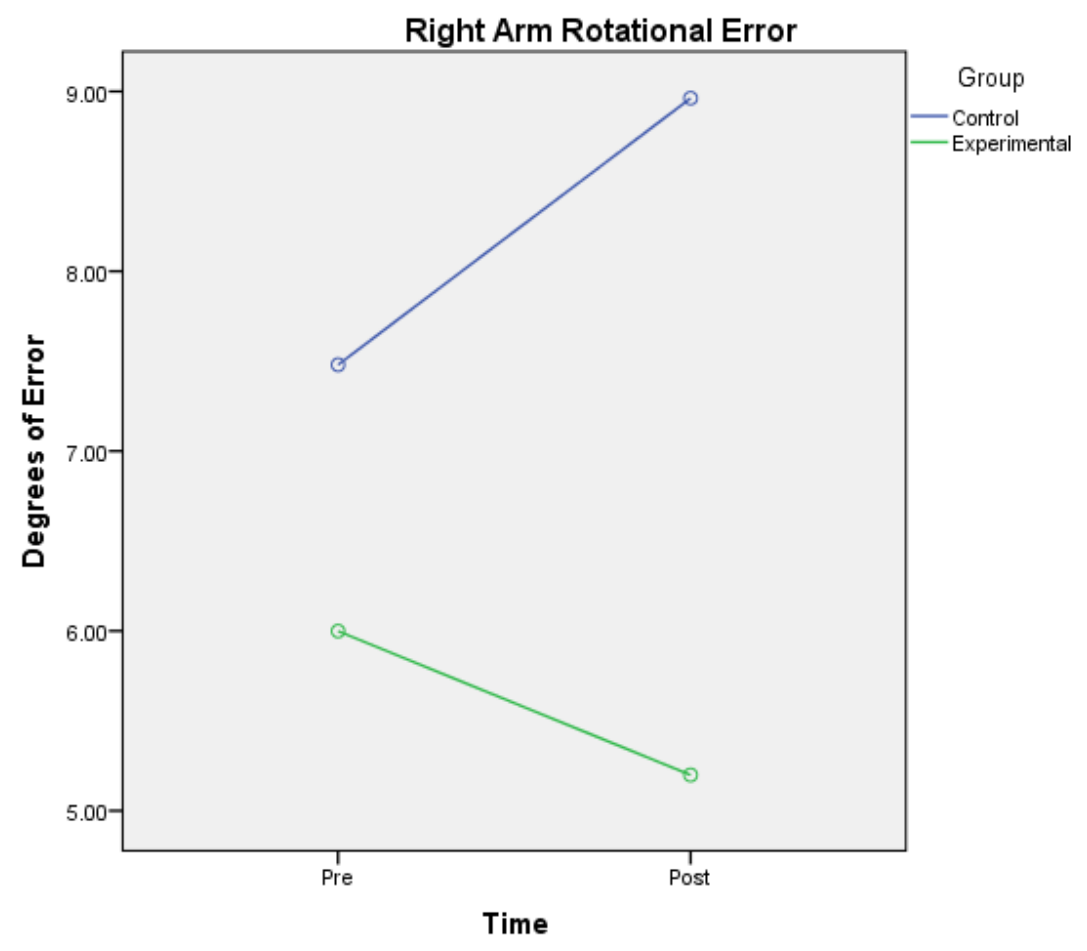


Figure D3. Functional throwing performance index plot

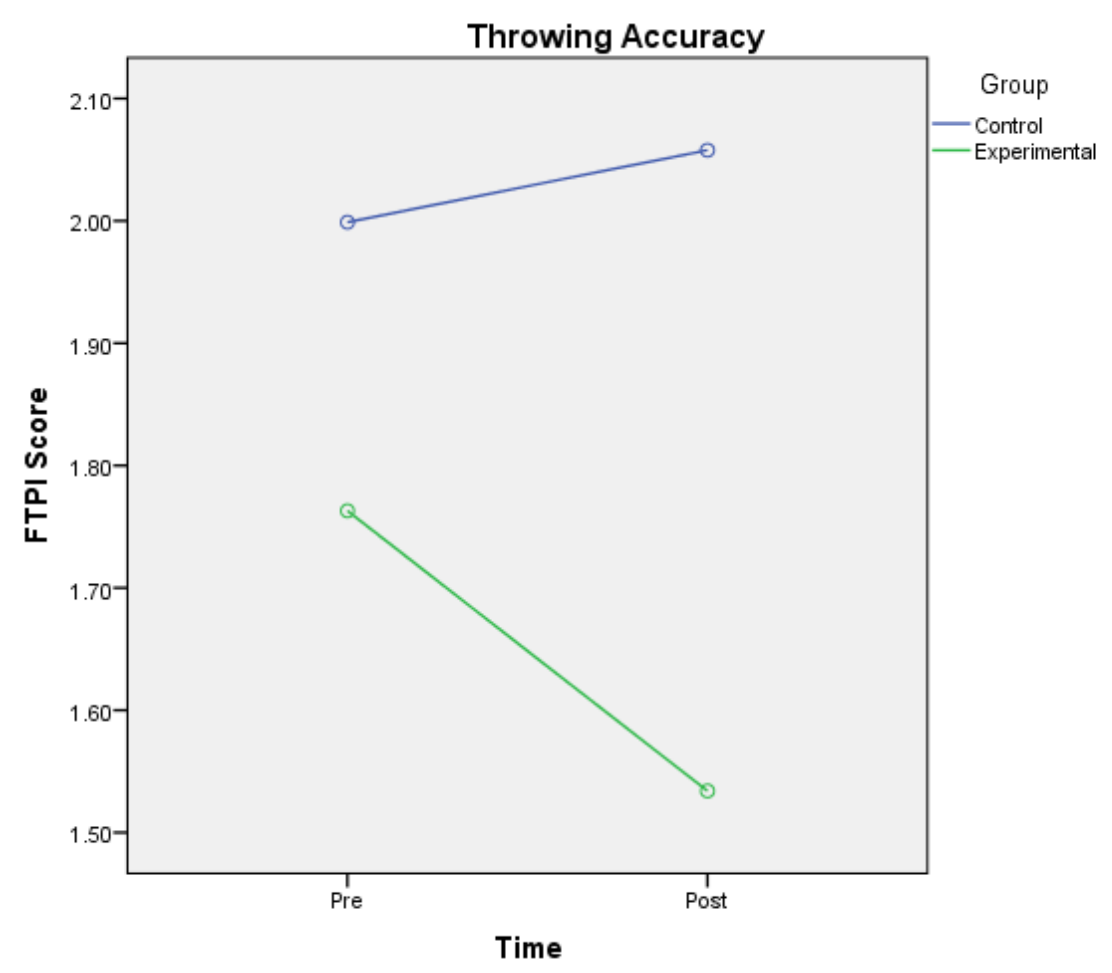

Figure D4. Throwing velocity plot

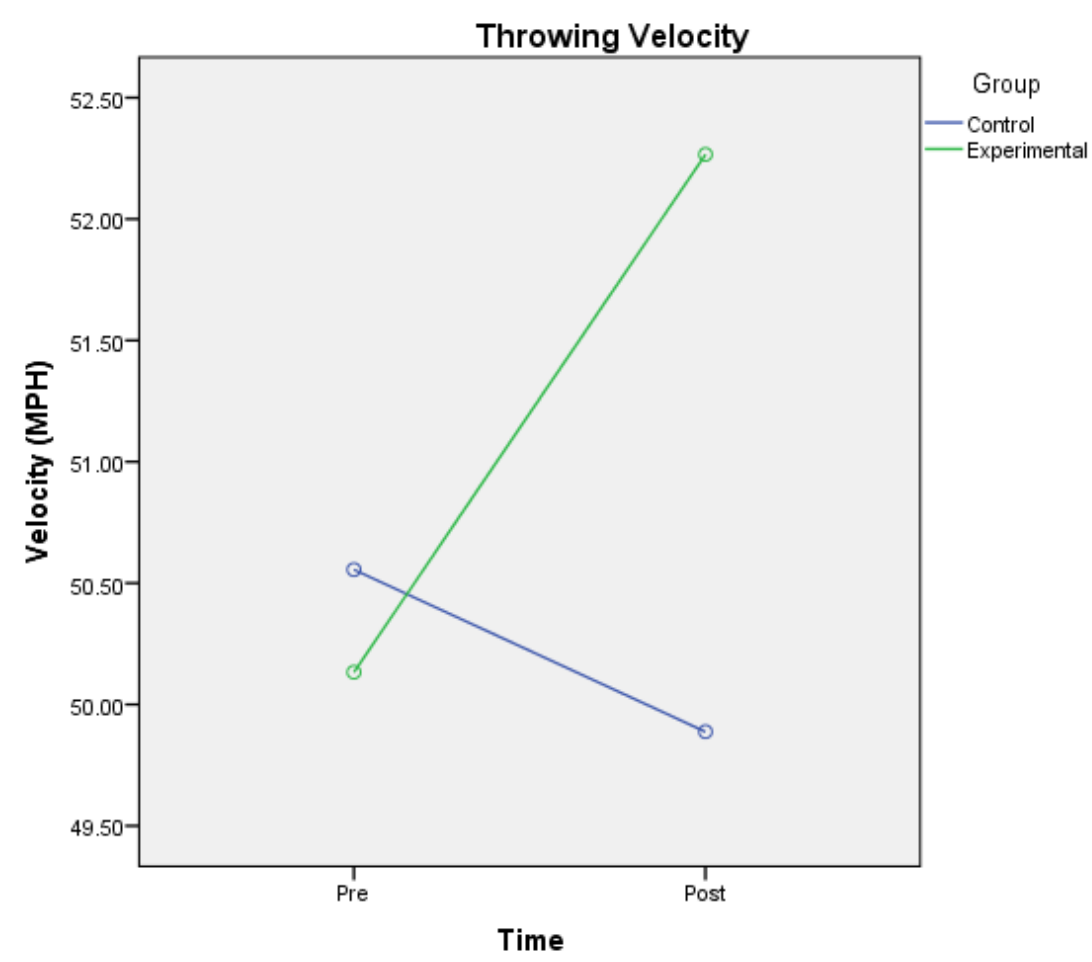


Figure D5. Closed kinetic chain upper extremity stability test plot

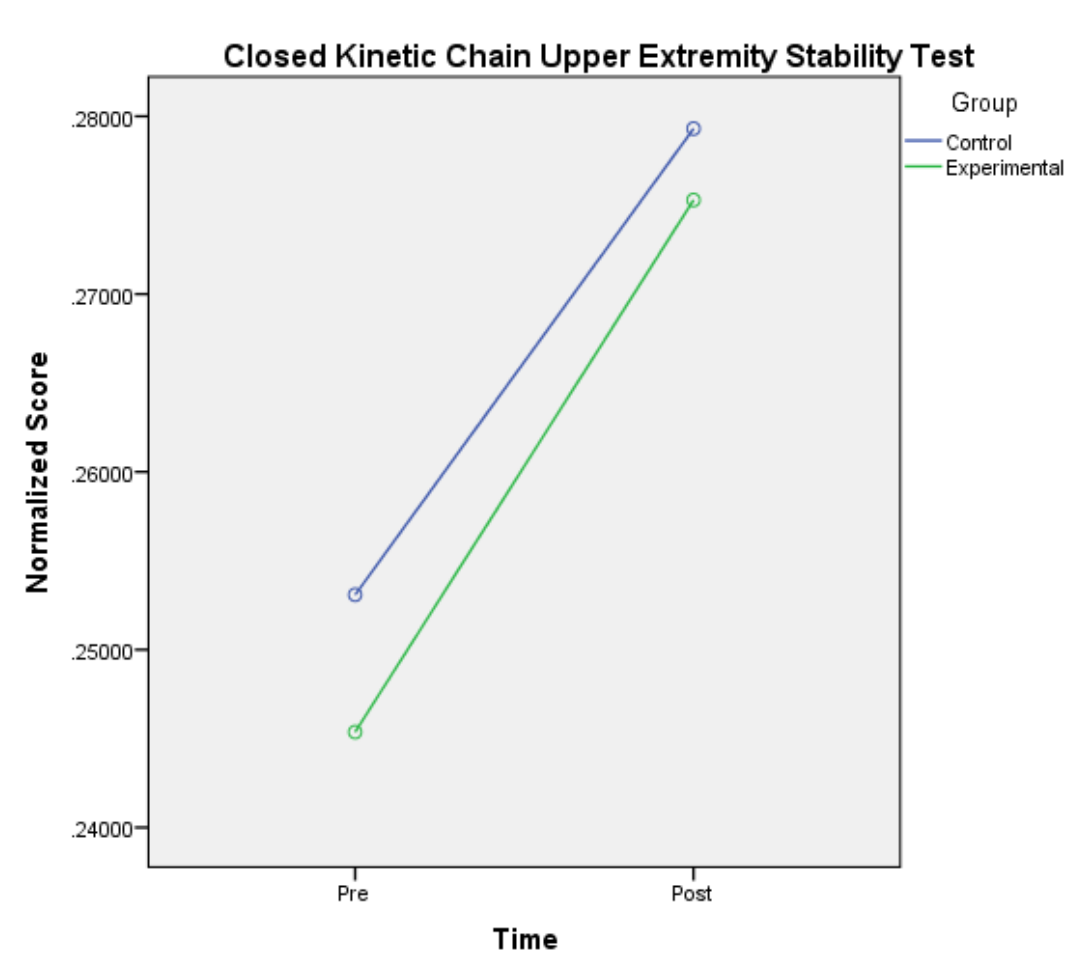




\section{APPENDIX E \\ RECOMMENDATIONS FOR FUTURE RESEARCH}

1. Increase the length of the study from six weeks to eight weeks

2. Increase the number of sessions to three times per week

3. Increase the sample size of participants

4. Use any overhead throwing athletes, including baseball players, football quarterbacks, and tennis athletes

5. Perform this intervention in the off-season

6. Utilize more multi-planar movement exercises earlier in the intervention

7. Perform a longitudinal study to examine the injury rates of those who participated in the intervention

8. Conduct the study using elite softball athletes

9. Conduct this study using injured athletes and use hit program in their return to play criteria

10. Create an intervention program specific to the positions that the subjects play

11. Use performance tests that are more specific to the typical throws of a position

12. Test joint position sense using glenohumeral joint complex abduction, internal rotation, external rotation, flexion, horizontal adduction and extension

13. Perform the joint position sense testing using a more accurate tool such as a biodex 


\section{ADDITIONAL REFERENCES}

33. Baechle T, Earle R. Essentials of Strength Training and Conditioning:National Strength and Conditioning Association. 2nd ed. Champaign, IL: Human Kinetics; 2000:20-21, 436-437, 404-407.

34. Tippett Steven. Functional Progressions for Sport Rehabilitation. Champaigne: Human Kinetics, 1995. Print.

35. Hibbs A, Thompson K, French D, Wrigley A, Spears I. Optimizing performance by improving core stability and strength. Sports Med. 2008;30(12):995-1008

36. Nesser T, Huxel K, Tincher J, Okada T. The relationship between core stability and performance in division-I football players. J Strength Cond Res. 2008;22(6):1750-1754

37. McGill S. Core training: evidence translating to better performance and injury prevention. Strength Cond J. 2010;32(3):33-46

38. Kibler B, Press J, Sciascia A. The role of core stability in athletic function. Sports Med. 2006;36(3):189-198

39. Willardson J. Core stability training: applications to sports conditioning programs. $J$ Strength Cond Res. 2007;21:979-85

40. Cissik J. The role of core training in athletic performance, injury prevention, and injury treatment. Strength Cond J. 2011;33(1):10-15

41. Schilling J. The role of the anatomical core in athletic movements. Int J Athl Ther Train. 2012;17(4):14-17

42. Reed C, Ford K, Myer G, Hewett T. The effects of isolated and integrated 'core stability' training on athletic performance measures. Sports Med. 2012;42(8):697-706

43. Voight M, Hardin J, Blackburn T, Tippett S, Canner G. The effects of muscle fatigue on and the relationship of arm dominance to shoulder proprioception. J Orthop Sport Phys Ther. 1996;23:348-52

44. Carpenter J, Blaiser R, Pellizzon G. The effects of muscle fatigue on shoulder joint position sense. Am J Sport Med. 1998;26:262-5

45. Chang H, Chen C, Wei S, Huang C. Recovery of joint position sense in the shoulder after muscle fatigue. J Sport Rehabil. 2006;15:312-25

46. Fleisig G, Andrews J, Dillman C, Escamilla R. Kinetics of baseball pitching with implications about injury mechanisms. Am J Sport Med. 1995;23:233-39 
47. Hurd W, Kaufman K. Glenohumeral rotational motion and strength and baseball pitching mechanics. J Athl Train. 2012;47:247-256

48. Ebben W, Hintz M, Simenz C. Strength and conditioning practices of major league baseball strength and conditioning coaches. J Strength Cond Res. 2005;19:538-546

49. Laudner K, Stanek J, Meister K. The relationship of periscapular strength on scapular upward rotation in professional baseball pitchers. J Sport Rehabil. 2008;17:95-105

50. Marshall P, Sci P, Murphy B. Core stability exercises on and off a swiss ball. Arch Phys Med Rehabil. 2005;86:242-248

51. Parkhouse K, Ball N. Influence of dynamic versus static core exercises on performance in field based fitness tests. J Body Mov Ther. 2011;15:517-524

52. Keogh J, Aickin S, Oldham R. Can common measure of core stability distinguish performance in a shoulder pressing task under stable and unstable conditions? J Strength Cond Res. 2010;24(2):422-429

53. Markovic G, Mikulic P. Neuro-musculoskeletal and performance adaptations to lower extremity plyometric training. Sports Med. 2010;40:859-895 